\title{
Problemi al contorno con condizioni omogenee per le equazioni quasi-ellittiche.
}

\author{
Mario Troisi (Bari) (*) (**)
}

Summary. - We are concerned with non-variational boundary value problems, with omogeneus boundary conditions, for linear partial differential equations of quasi-elliptic type in a bounded domain $\Omega$ in $R^{n}$.

It is well known that some of difficulties which arise in treating such problems, in comparison with "regular, elliptic problems, are connected with the presence of angular points in 8 : let us point out with B. PrNI [32] that * a bounded domain for which it is possible to assign a correct boundary value problem for a quasi-elliptic but not elliptic equation always has angular points.

We suppose $\Omega$ is a cartesian product of a finite number of open sets and, in order to overcome the difficulties attached to the presence of angular points in $\Omega$, taking as a model the two previous papers [33], [34] devoted to elliptic problems with singular data, we investigate the problem within suitable Sobolev weight spaces, connected with the angular points of $\mathrm{Q}$ and included in the ones we have studied in [35]. Within such spaces we get existence and uniqueness theorems.

Per dare, con qualche esempio, un'idea dei problemi che studiamo e dei risultati che otteniamo, assegnamo nel piano il rettangolo $\Omega=\{(x, y) \mid$ $a<x<b, o<y<d \mid$, di cui indichiamo con $\Gamma_{1}$ la porzione di frontiera unione dei due lati di equazioni $x=a$ e $x=b$, con $\Gamma_{2}$ la porzione di frontiera unione dei due lati di equazioni $y=c$ e $y=d$.

Indichiamo, inoltre, con $p_{0}(x, y)$ la distanza di $(x, y)$ dai vertici di $\Omega$, con $\rho_{1}(x, y)$ la distanza di $(x, y)$ da $\Gamma_{1}$, ed assegnamo $k \in\{0,1\}, s \in R^{1}$ e $f(x, y)$ tale che $\rho_{k}^{s} f \in L^{2}(\Omega)$.

Consideriamo allora il problema consistente nel determinare una solu. zione $u(x, y)$ dell' equazione

tale che

$$
\frac{\partial^{4} u}{\partial x^{4}}-\frac{\partial^{2} u}{\partial y^{2}}+\lambda u=f \text { in } \Omega \quad(\lambda=\text { costante })
$$

$$
\iint_{\Omega}\left(\left.\left|\rho_{k}^{s-4} u_{\mid}^{2}+\right| \rho_{k}^{s} \frac{\partial^{4} u}{\partial x^{4}}\right|^{2}+\left|\rho_{k}^{s} \frac{\partial^{2} u}{\partial y^{2}}\right|^{2}\right) d x d y<\infty
$$

(*) Lavoro eseguito con contributo del C.N.R.

(**) Entrata in Redazione il 30 ottobre 1971. 
e che nel caso $k=0$ soddisfi le condizioni

$$
u=\frac{\partial u}{\partial x}=0 \text { su } \Gamma_{1}, u=0 \text { su } \Gamma_{2},
$$

mentre nel caso $k=1$ soddisfi la condizione

$$
u=0 \text { su } \mathrm{C}_{2} \text {. }
$$

Dai nostri risultati segue che esiste $\eta_{0} \in$ ] $0,1 / 2$ [ tale che, se $s \in\left[2-\eta_{0}\right.$, $2+\eta_{0}$, per tale problema vale l'alternativa di Fredhocm e si ha univoca risolubilità per $\lambda$ sufficientemente grande.

Indichiamo ancora con $\rho_{2}(x, y)$ la distanza di $(x, y)$ da $\mathrm{T}_{2}$, con $\rho_{3}(x, y)$ la distanza di $(x, y)$ da $\partial \Omega$, assegnano $k \in\{0,1,2,3\}, s \in R^{1}, f(x, y)$ come sopra, e consideriamo il problema consistente nel determinare una soluzione $u(x, y)$ dell' equazione

$$
\frac{\partial^{4} u}{\partial x^{4}}-\frac{\partial^{2} u}{\partial y^{2}}+\lambda \rho_{k}^{-4} u=f \text { in } \Omega \quad(\lambda=\text { costante }),
$$

che verifichi la (1) e che: nel caso $k=0$ soddisfi le condizioni

$$
\frac{\partial u}{\partial x}=\frac{\partial^{3} u}{\partial x^{3}}=0 \text { su } \Gamma_{1}, \quad \frac{\partial u}{\partial y}=0 \text { su } \Gamma_{2},
$$

nel caso $k=1$ soddisfi la condizione

$$
\frac{\partial u}{\partial y}=0 \mathrm{su} \Gamma_{2}
$$

nel caso $k=2$ soddisfi le condizioni

$$
\frac{\partial u}{\partial x}=\frac{\partial^{3} u}{\partial x^{3}}=0 \mathrm{su} \Gamma_{1}
$$

Dai nostri risultati segue che, qualunque sia $s \in R^{1}$, esiste una costante $\lambda_{s}>0$ tale che per ogni $\lambda \geq \lambda_{s}$ detto problema risulta univocamente risolubile.

In generale, siano: $n_{1}, \ldots, n_{t}$ dei numeri interi positivi tali che $n_{1}+\ldots$ $+n_{t}=n, m_{1}, \ldots, m_{n}$ dei numeri interi positivi tali che $m_{n_{0}}+\ldots+n_{i-1}+1=\ldots$ $=m_{n_{0}+\ldots+n_{i}}=m^{(i)}(i=1, \ldots, t)$, dove $n_{0}=0$.

Poniamo $m=\sup \left\{m_{1}, \ldots, m_{n}\right\}, q_{i}=m / m_{i}, q=\left(q_{1}, \ldots, q_{n}\right\},\langle\alpha, q\rangle=$ $=\alpha_{1} q_{1}+\ldots+\alpha_{n} q_{n}$. 
Assegnamo, per ogni $i \in\{1, \ldots, t\}$, un insieme aperto e limitato $\Omega^{(i)} \mathrm{di}$ $R^{n_{i}}$ e un insieme chiuso $S^{(i)}$, eventualmente vuoto, di punti di $\partial \Omega^{(i)}$, con la condizione che, se $S^{(i)} \neq \partial \Omega^{(i)}, \partial \Omega^{(i)}-S^{(i)}$ sia una varietà a $n_{i}-1$ dimensioni di classe $C^{\infty}$ e $\Omega^{(i)}$ sia situato localmente da una stessa parte di $\partial \Omega^{(i)}-S^{(i)}$.

Poniamo $\Omega=\Omega^{(1)} \times \ldots \times \Omega^{(i)}, \partial_{i} \Omega=\Omega^{(1)} \times \ldots \times \Omega^{(i-1)} \times\left(a \Omega^{(i)}-S^{(i)}\right) \times$ $\times \Omega^{(i+1)} \times \ldots \times \Omega^{(i)}(i=1, \ldots, t), S=\partial \Omega-\bigcup_{i=1}^{t} \partial_{i} \Omega, \rho(x)=\delta$ dist $(x, S)$, dove $\delta$ è un'opportuna costante positiva, e indichiamo con $\mathfrak{J}$ l'insieme dei numeri $i \in\{1, \ldots, t\}$ per cui $\partial_{i} \Omega \neq \varnothing$.

Inoltre indichiamo con $L_{s}^{2}(\Omega), s$ reale, la classe delle funzioni $u$ tali che $p^{s} u \in L^{2}(\Omega)$ e con $W_{s}^{m}(\Omega)$ la classe delle distribuzioni $u$ su $\Omega$ tali che $D^{x} u \in L_{s+<\alpha, q>-m}^{2}(\Omega)$ per $\langle\alpha, q>\leq m$.

Assegnamo in $\Omega$ un operatore differenziale lineare $A=\underset{<x, q>\leq m}{\Sigma} a_{x}(x) D^{a}$ di tipo quasi-ellittico e, per ogni $i \in \mathfrak{J}$, propriamente quasi-ellittico di tipo $v_{i}$ su $\partial_{i} \Omega$ (cfr. il n. 8 , ipotesi $\left.\mathscr{H}_{2}\right)$ ).

Assegnamo inoltre, per ogni $i \in \mathfrak{J}$, un sistema $\left\{B_{i j}\right\}_{j=1}^{\nu_{i}}$ di $v_{i}$ operatori dif. ferenziali lineari di frontiera definiti su $\partial_{i} \Omega$, della forma

$$
B_{i j}=\sum_{<\alpha, q>\leq p_{i j}} b_{j \alpha}^{(i)}(x) D^{\alpha}, \quad p_{i j} \leq m-m / m^{(i)},
$$

e soddisfacenti la condizione complementare rispetto ad $A$ su $\partial_{i} \Omega$ (cfr. il n. 8 , ipotesi $\left.\mathfrak{H H}_{4}\right)$ ).

Studiamo allora il problema

$$
A u=f \text { in } \Omega, \quad B_{i j} u=0 \text { su } \partial_{i} \Omega, j=1, \ldots, v_{i}, i \in \mathfrak{J},
$$

cercando la soluzione negli spazi $W_{s}^{m}(\boldsymbol{\Omega})$.

I risultati che otteniamo vanno distinti in quelli relativi al caso in cui le condizioni al contorno sono di tipo generale ed in quelli relativi al caso in cui le condizioni al contorno sono del tipo di DrRICHLET, e ciò̀ al caso in oui $B_{i j}=\left(\partial / \partial n_{i}\right)^{i}$, dove $n_{i}$ è la normale a $\partial_{i} \Omega$.

Nel primo caso otteniamo dei teoremi di unicità e di esistenza, richiedendo però ohe nell'equazione almeno il coefficiente di $u$ sia singolare su $S$ oppure che la quasi-ellitticità di $A$ degeneri su $S$.

Nel secondo caso, e nell' ipotesi che $A$ sia fortemente quasi-ellitlico in $\bar{\Omega}$, otteniamo ulteriori teoremi di unicità e di esistenza sotto ipotesi meno generali sui coefficienti di $A$ ma in eui viene attenuata od eliminata la precedente ipotesi di singolarità del coefficiente di $u$.

Rileviamo anche, come in [33], che, sebbene nei problemi considerati nessuna condizione al contorno sia imposta a priori su $S$, la soluzione può soddisfare su $S$ ad un certo numero di condizioni omogenee del tipo di Diriohlet, 
come condizioni naturali derivanti dall'appartenenza della soluzione alla classe $W_{s}^{m}(\Omega)$. Infatti dal lemma 7.8 segue che se $u \in W_{s}^{m}(\Omega)$ e $D^{x} u$ è dotata di traccia su $S$, tale traccia è nulla.

Il lavoro è suddiviso in tre capitoli.

Il Cap. I, di carattere preliminare ed i cui risultati trovano applicazioni nei teoremi esistenziali stabiliti negli altri capitoli, ò dedicato a questioni di regolarizzazione per problemi quasi-ellittici nel semispazio ed a questioni concernenti la formula di GREeN nel semispazio per operatori quasi-ellittici.

Nel n. 1 sono introdotti aleuni spazi di distribuzioni che rientrano in spazi più generali studiati da vari Autori, fra i quali ricordiamo L. Hörmander [18], L. R. Volevich-B. P. Paneyakh [37], A. Cavalludcoi [10], M. ITano [19]. Ne vengono richiamati alcuni teoremi di densità, di inclusione e di tracce su iperpiani.

Nei nn. 2 e 3 vengono stabilite delle limitazioni a priori nel semispazio relative ad operatori quasi-ellittici. Tali limitazioni sono analoghe ad altre note nel caso ellittico e contenute, ad es., nei nn. 4.4 e 4.5 del cap. 2 di J. L. Lions-E. MAGenes [20]. Le loro dimostrazioni si ottengono opportunamente adattando al nostro caso i procedimenti di [20].

Nei nn. 4 e 5 vengono stabiliti dei teoremi di regolarizzazione per operatori quasi-ellittici. La tecnica della dimostrazione del teorema 4.1 di rego. larizzazione tangenziale è in parte del tipo di quella utilizzata da T. Matsuzawa in [21] per dimostrare un altro risultato. La dimostrazione del teorema 5.1 si ottiene adattando al nostro caso un ben noto procedimento relativo al caso ellittico.

Nel n. 6 vengono stabiliti un'opportuna formula di GREEN per il semispazio ed alcuni risultati ad essa connessi. Tali risultati trovano applicazioni al n. 11 nel teorema relativo alla formula di GreEN in un dominio limitato.

Il Cap. II è dedicato allo studio dei problemi al contorno quasi-ellittici in un dominio limitato e nell' ambito degli spazi $W_{s}^{m}(\Omega)$.

Nel n. 7 si introducono gli aperti $\Omega$ e si approfondisce lo studio degli spazi $W_{s}^{m}(\Omega)$, metrendone in lace alcuni teoremi di densità e di immersione, in parte dedotti dai risultati del precedente lavoro [35].

Nel n. 8 vengono precisate le ipotesi sugli operatori che intervengono nel problema e vengono enunciati i risultati, che sono nn opportuno teorema di regolarizzazione, un teorema di unicitả ed un teorema di esistenza.

Tali risultati costituiscono un'estensione di altri risultati da noi stabiliti nel caso ellittico (cfr. [33] e [34]), e le loro dimostrazioni, che sono date nei nn. 9, 10 e 11, si ottengono con procedimenti analoghi a quelli tenuti in [33], [34] ed atilizzando una limitazione a priori stabilita da T. Matsuzawa in [21].

Il Cap. III è dedicato ad un ulteriore approfondimento dello studio del problema considerato nel Cap. II nel caso particolare che $A$ sia fortemente quasi-ellittico in $\bar{\Omega}$ e che i dati al contorno siano quelli di Dirichlet. 
$\mathrm{Nel}$ n. 12 vengono studiati alcuni spazi funzionali contenuti in quelli considerati al n. 7 e vengono stabilite delle limitazioni a priori relative a funzioni di tali spazi e ad operatori fortemente quasi-ellittici in $\bar{\Omega}$.

Nei nn. 13 e 14 vengono stabiliti dei teoremi di unicità e dei teoremi di esistenza specifici per il problema di DIRICHLET e che non rientrano in quelli del Cap. II.

Rileviamo che questi ultimi risultati non solo estendono al caso quasiellittico i risultati contenuti nei nn. 6 e 7 di [33], ma sono di questi più generali anche se riferiti ad equazioni ellittiche.

\section{Capitolo I}

\section{Problemi quasi-ellittici in un semispazio.}

\section{1. - Aleuni spazi di distribuzioni.}

Indicheremo con $R^{n}$ lo spazio reale euclideo a $n$ dimensioni di punto $x=\left(x_{1}, \ldots, x_{n}\right)$ e con $R_{+}^{n}$ il semispazio $\left\{x \in R^{n} \mid x_{n}>0\right\}$.

Se $\alpha=\left(\alpha_{1}, \ldots, \alpha_{n}\right)$ è una $n$-pla di interi non negativi, porremo

$$
\begin{gathered}
|\alpha|=\alpha_{1}+\ldots+\alpha_{n}, x^{\alpha}=x_{1}^{\alpha_{1}} \ldots x_{n}^{\alpha_{n}} \\
D^{\alpha}=D_{x_{1}}^{\alpha_{1}} \ldots D_{x_{n}}^{\alpha_{n}}
\end{gathered}
$$

dove

$$
D_{x_{k}}=\frac{1}{i} \frac{\partial}{\partial x_{k}}, k=1, \ldots, n, \quad i=\sqrt{-1}
$$

Porremo anche

$$
\begin{gathered}
x=\left(x^{\prime}, t\right), x^{\prime}=\left(x_{1}, \ldots, x_{n-1}\right), t=x_{n}, \\
\alpha=\left(\alpha^{\prime}, \alpha_{n}\right), \alpha^{\prime}=\left(\alpha_{1}, \ldots, \alpha_{n-1}\right), D_{x^{\prime}}^{\alpha^{\prime}}=D_{x_{1}}^{\alpha_{1}} \ldots D_{x_{n-1}}^{\alpha_{n-1}} .
\end{gathered}
$$

Denoteremo con $\tilde{u}(\xi)$ la trasformata di Fourier di $u(x)$, con $\hat{u}\left(\xi^{\prime}, t\right)$ la la trasformata parziale di Fourier di $u(x)=u\left(x^{\prime}, t\right)$ rispetto a $x^{\prime}$ e con $\widehat{g}\left(\xi^{\prime}\right)$ la trasformata di Fourien di $g\left(x^{\prime}\right)$.

Assegnamo una $n$-pla di interi positivi $\mathfrak{T} \mathcal{C}=\left(m_{1}, \ldots, m_{n}\right)$. 
Porremo

$$
\begin{aligned}
& m=\sup \left\{m_{1}, \ldots, m_{n}\right\}, d=\text { m.c.d. }\left\{m_{1}, \ldots, m_{n}\right\}, m_{0}=m / d, \\
& q_{k}=m / m_{k}(k=1, \ldots, n), q=\left(q_{1}, \ldots, q_{n}\right),|q|=q_{1}+\ldots+q_{n} .
\end{aligned}
$$

Per ogni coppia di punti $x, y \in R^{n}$ porremo

$$
<x, y>=x_{1} y_{1}+\ldots+x_{n} y_{n} .
$$

Inoltre per ogni $\xi=\left(\xi_{1}, \ldots, \xi_{n}\right)=\left(\xi^{\prime}, \xi_{n}\right) \in R^{n}$ porremo

$$
<\xi>=\left(\sum_{k=1}^{n}\left|\xi_{k}\right|^{m_{k}}\right)^{1 / m},\left\langle\xi^{\prime}>=\left(\sum_{k=1}^{n-1}\left|\xi_{k}\right| m_{k}\right)^{1 / m} .\right.
$$

Si verifica facilmente che, comunque si assegna un numero reale $a$, si ha

$$
\left(\frac{1+<\xi>^{2}}{1+<\eta>^{2}}\right)^{a} \leq c\left(1+|\xi-\eta|^{2|a|}\right) \forall \xi, \eta \in R^{n},
$$

dove $c$ è una costante dipendente solo da $a$, פK ed $n$.

Deminizione 1.1. - Indicheremo con $H^{s, r}\left(R^{n}\right), s$ e $r$ reali, lo spazio delle distribuzioni temperate $u$ su $R^{n}$ tali che

$$
\left(1+<\xi>^{2}\right)^{s / 2}\left(1+<\xi^{\prime}>^{2}\right)^{r / 2} \tilde{u}(\xi) \in L^{2}\left(R^{n}\right),
$$

munito della norma

\section{Porremo}

$$
\|u\|_{H^{s, r}\left(R^{n}\right)}=\left(\int_{R^{n}}\left(1+<\xi>^{2}\right)^{s}\left(1+<\xi^{\prime}>^{2}\right)^{r}|\tilde{u}(\xi)|^{2} d \xi\right)^{1 / 2} .
$$

$$
H^{s}\left(R^{n}\right)=H^{s \cdot 0}\left(R^{n}\right)
$$

Osserviamo ohe, in conseguenza della (1.1), gli spazi $H^{s, r}\left(R^{n}\right)$ sopra introdotti rientrano in spazi di distribuzioni più generali studiati da diversi Autori, fra i quali ricordiamo L. Hörmander [18], L. R. Volevich-B. P. Paneyakh [37].

Dai risultati contenuti nei succitati lavori traiamo il seguente

LEMMa 1.1. - $O_{0}^{\infty}\left(R^{n}\right) \grave{e ̀ ~ d e n s o ~ i n ~} H^{s, r}\left(R^{n}\right)$. Risulta $H^{s, r}\left(R^{n}\right)=\left(H^{-s,-r}\left(R^{n}\right)\right)^{\prime}\left({ }^{1}\right)$. Se $s_{2} \leq s_{1}$ e $s_{2}+r_{2} \leq s_{1}+r_{1}$, allora si ha $H^{s_{1}, r_{2}}\left(R^{n}\right) \subseteq H^{s_{2}, r_{2}}\left(R^{n}\right)$ algebricamente e topologicamente.

(1) Se $E$ è uno spazio di Banach, indicheremo con $E^{\prime}$ il duale forte di $E$. 
Osserviamo anche, come si verifica facilmente, che:

Lemma 1.2. - Se $s_{1}>s_{2}>s_{3}$, allora per ogni $\varepsilon>0$ esiste una costante positiva $c_{\varepsilon}=c\left(\varepsilon, s_{1}, s_{2}, s_{3}\right)$ tale che si $h a$

$$
\|u\|_{H} s_{2, r}\left(R^{n}\right) \leq \varepsilon\|u\|_{H H^{s_{1}}, r} r_{\left(R^{n}\right)}+c_{\varepsilon}\|u\|_{H H^{s_{3}, r}\left(R^{n}\right)}
$$

per ogni $r$ reale e per ogni $u \in H^{s_{1}, r}\left(R^{n}\right)$.

Sussiste inoltre il seguente

Lama 1.3. - Siano u una distribuzione di classe $H^{s, r}\left(R^{n}\right)$ ed a supporto compatto, $\mathfrak{D}$ un insieme aperto e limitato contenente il supporto $d i$ u $e$ a(x) una funzione di classe $C^{\infty}\left(R^{n}\right)$.

Esiste una costante positiva $c$, indipendente da $u$, tale che si ha

$$
\left.\|a u\|_{H^{s, r}\left(R^{n}\right)} \leq \sup _{x \in \mathfrak{D}}|a(x)| \cdot\|u\|_{H^{s, r}\left(R^{n}\right)}+c\|u\|_{H^{s_{1} r-1}}{ }_{\left(R^{n}\right)}\right)
$$

dove, se $r=0$, l'ultima norma pù̀ essere sostituita dalla norma di $u$ in $H^{s-1}\left(R^{n}\right)$.

Infatti, consideriamo una funzione $\zeta(x) \in C_{0}^{\infty}\left(R^{n}\right)$ tale che

$$
0 \leq \zeta(x) \leq 1, \zeta(x)=1 \text { in supp } u, \operatorname{supp} \zeta \subset \mathscr{D},
$$

e poniamo $b(x)=\zeta(x) a(x)$.

Con ragionamenti analoghi a quelli tenuti da J. PeETre per stabilire il lemma 2 di [30], si dimostra che esiste una costante $c$ (indipendente da $u$ ) tale che

$$
\|b u\|_{H^{s, r}\left(R^{n}\right)} \leq \sup _{x \in R^{n}}|b(x)| \cdot\|\boldsymbol{u}\|_{H^{s, r}\left(R^{n}\right)}+c\|u\|_{H^{s, r-1}\left(R^{n}\right)}
$$

dove, se $r=0$, l'ultima norma può essere sostituita dalla norma di $u$ in $H^{s-1}\left(R^{n}\right)$.

Da quest' ultima relanione si deduce in modo ovvio la tesi.

Denoteremo con $J(\mathscr{N})$ l'insieme dei numeri razionali $k$ tali che l'equazione

$$
<\alpha, q>=k
$$

abbia almeno una soluzione $\alpha=\left(\alpha_{1}, \ldots, \alpha_{n}\right)$, con gli $\alpha_{i}$ interi $\geq 0$.

Defintzione 1.2. - Se $\mathscr{D}$ è un insieme aperto di $R^{n}$, indicheremo $W^{k, p}(\mathfrak{D})$, $k \in J(\mathscr{O K})$ e $p$ reale $\geq 1$, lo spazio delle distribuzioni $u$ su $\mathscr{D}$ tali che $D^{\alpha} u \in L^{p}(\mathfrak{D})$ per $<\alpha, q>\leq k$, munito della norma

$$
\|u\|_{W^{k}, p(\mathfrak{D})}=\underset{<\alpha, q>\leq k}{\Sigma}\left\|D^{\alpha} u\right\|_{L P(\mathfrak{D})}
$$


Porremo $W^{k}(\mathfrak{D})=W^{k, 2}(\mathfrak{D})$.

LeMra 1.4. - Se $k \in J(\mathfrak{O C})$, esistono due costanti $c_{1}(k)$ e $c_{2}(k)$ tali che si ha per ogni $\xi \in R^{n}$

$$
c_{1}(k)<\xi>^{2 k} \leq \sum_{<\alpha, q>=k_{k}}^{\Sigma} \xi^{2 \alpha} \leq c_{2}(k)<\xi>^{2 k}
$$

dove, se $k$ è un multiplo di $m_{0}$, si può supporre $c_{1}(k)>0$.

Inoltre il sussistere della (1.6) con $c_{1}(k)>0$ e $\xi$ fuori di un compatto di $R^{n}$ implica che $k$ è un multiplo di $m_{0}$.

Infatti, si stabilisce la (1.6) per ogni $\xi \in R^{n}$, osservando che $\xi^{2 \alpha} \geqslant 0$ e che si ha

$$
\left.\xi^{2 \alpha}=\left(\prod_{i=1}^{n}\left(\left|\xi_{i}\right| m_{i}\right)^{\alpha_{i} q_{i}|<\alpha, q\rangle}\right)\right)^{2<\alpha, q>\mid m} \leq\left\langle\xi>^{2<\alpha, q\rangle} .\right.
$$

Se poi $k$ è multiplo di $m_{0}$, i numeri $m_{i} k / m$ sono interi e quindi il poli. nomio $\sum_{\langle\alpha, q>=k} \xi^{2 \alpha}$ è minorato dal polinimio $\sum_{i=1}^{n} \xi_{i}^{2 m_{i}{ }^{k / m}}$ e perciò anche dal primo membro della (1.6) con una costante $c_{1}(k)$ positiva.

Per dimostrare l'ultima affermazione, osserviamo che, se è verificata la (1.6) con $c_{1}(k)>0$ e per $\xi$ fuori di un compatto di $R^{n}$, esiste $a>0$ tale che per ogni $t \in] a, \infty[$ si ha

$$
\frac{\sum_{r=0}^{\left[m_{i} k / m\right]} t^{2 r}}{t^{2 m_{i} k / m}} \geq c_{1}(k)>0, \quad i=1, \ldots, n,
$$

dove $\left[m_{i} k / m\right]$ denota il più grande intero $\leq m_{i} k / m$.

Ne segue che i numeri $m_{i} k / m, i=1, \ldots, n$, sono interi e quindi che $k$ è un multiplo di $m_{0}$. 
Lemma 1.5. - I due spazi $W^{k}\left(R^{n}\right)$ e $H^{k}\left(R^{n}\right)$ sono isomorfi (algebricamente e topologicamente) se e solo se $k$ è un multiplo di $m_{0}$.

Infatti, osserviamo che, se $k$ è un multiplo di $m_{0}$, in conseguenza del lemma 1.4 esiste una costante $c=c(k)>0$ tale che si ha

$$
c^{-1}\left(1+<\xi>^{2}\right)^{k} \leq \sum_{<x, q>\leq k} \xi^{2 x} \leq c\left(1+<\xi>^{2}\right)^{k} \quad \forall \xi \in R^{n}
$$

Osserviamo inoltre che, se è verificata la (1.8), risulta anche verificata la (1.6) con $c_{1}(k)>0$ e per $\langle\xi>$ sufficientemente grande, e quindi, ancora per il lemma $1.4, k$ risulta un multiplo di $m_{0}$.

Da tali osservazioni si deduce la tesi con note considerazioni.

Definizione 1.3 - Indicheremo con $H^{s, r_{(}}\left(R_{+}^{n}\right), s$ e $r$ reali, lo spazio delle distribuzioni $u$ su $R_{+}^{n}$ che sono restrizioni a $R_{+}^{n}$ di elementi di $H^{s, r}\left(R^{n}\right)$, munito della norma

$$
\|u\|_{\left.H^{s}, r_{(} R_{+}^{n}\right)}=\text { inf }\|U\|_{H^{s,}, r_{\left(R^{n}\right)}}, \quad U=u \quad \text { su } \quad P_{+}^{n}
$$

Dal lemma 1.1 si deduce, con note considerazioni (efr., ad es., il cap. I di L. R. Volevich - B. P. PanexakH [37]), il seguente

LeMma 1.6. - $C_{0}^{\infty}\left(\overline{R_{+}^{n}}\right)$ è denso in $H^{s, r}\left(R_{+}^{n}\right)$. Se $s_{2} \leq s_{1}$ e $s_{2}+r_{2} \leq s_{1}+r_{1}$, allora si ha $H^{s_{1} \cdot r_{1}}\left(R_{+}^{n}\right) \subseteq H^{s_{2}, r_{2}}\left(R_{+}^{n}\right)$ algebricamente e topologicamente. $\left(H^{s, r}\left(R_{+}^{n}\right)\right)^{\prime} \grave{e}$ isomorfo algebricamente e topologicamente al sottospazio $\left.H_{R_{+}^{n}}^{\frac{-s_{*}}{R^{n}}}-R^{n}\right) d i$ $H^{-s,-r}\left(R^{n}\right)$ costituito dalle $u \in H^{-s_{2}-r}\left(R^{n}\right)$ che hanno supporto contenuto in $\overline{R_{+}^{n}}$.

Defintzione 1.4. - Indicheremo con $H^{s}\left(R^{n-1}\right), s$ reale, lo spazio delle distribuzioni temperate $g$ su $R^{n-1}$ tali che

$$
\left(1+<\xi^{\prime}>^{2}\right)^{s / 2} \widehat{g}\left(\xi^{\prime}\right) \in L^{2}\left(R^{n-1}\right)
$$

munito della norma

$$
\left\|\boldsymbol{g}^{\prime}\right\|_{H^{s}\left(R^{n-1}\right)}=\left(\int_{R^{n-1}}\left(1+<\xi^{\prime}>^{2}\right)^{s}\left|\bar{g}\left(\xi^{\prime}\right)\right|^{2} d \xi^{\prime}\right)^{1 / 2} .
$$


Per ogni numero reale $s>m / 2 m_{n}$, denoteremo con $l_{s}$ il più grande intero $<s m_{n} / m-1 / 2$. Inoltre per ogni $u \in C_{0}^{\infty}\left(\overline{R_{+}^{n}}\right)$ porremo

$$
\gamma_{j} u=D_{x_{n}}^{j} u\left(x^{\prime}, 0\right)
$$

Sussiste il seguente

LEMMA 1.7. - Siano $s>m / 2 m_{n}$ e $r$ due numeri reali. L'applicazione $u \rightarrow\left(\gamma_{0} u, \ldots, \gamma_{s} u\right)$ definita su $C_{0}^{\infty}\left(\overline{R_{+}^{n}}\right)$ si prolunga per continuità in un'applicazione, che ancora indichiamo con $u \rightarrow\left(\gamma_{0} u, \ldots, \gamma_{s} u\right)$, lineare e continua da $H^{s, r}\left(R_{+}^{n}\right) s u \prod_{j=0}^{l_{s}} H^{s+r-j q_{n}-q_{n}{ }^{2 / 2}\left(R^{n-1}\right) .}$

Infatti, poniamo

$$
\begin{gathered}
k(\xi)=\left(1+\sum_{i=1}^{n}\left|\xi_{i}\right|^{2 s m i_{i}^{\prime m}}\right)\left(1+<\xi^{\prime}>^{2}\right)^{r}, \\
k_{j}\left(\xi^{\prime}\right)=\left(\int_{-\infty}^{+\infty} \frac{\xi_{n}^{2 j}}{k(\xi)} d \xi_{n}\right)^{-1}, \quad j=0,1, \ldots, l_{s} .
\end{gathered}
$$

Si verifica facilmente che esiste una costante $c$ tale che si ha

$$
\begin{array}{cc}
c^{-1}\left(1+<\xi>^{2}\right)^{s}\left(1+<\xi^{\prime}>^{2}\right)^{r} \leq k(\xi) \leq c\left(1+<\xi>^{2}\right)^{s}\left(1+<\xi^{\prime}>^{2}\right)^{r} \quad & \forall \xi \in R^{n}, \\
c^{-1}\left(1+<\xi^{\prime}>^{2}\right)^{s+r-j q_{n}-q_{n}{ }^{2}} \leq k_{j}\left(\xi^{\prime}\right) \leq c\left(1+<\xi^{\prime}>^{2}\right)^{s+r-j q_{n}-q_{n}{ }^{2}} \quad \forall \xi^{\prime} \in R^{n-1} .
\end{array}
$$

Da tali relazioni segue, per noti risultati (cfr., ad es.: il teorema 2 e l'osservazione finale del n. 2 di A. CAVALLCCOI [10]; il teorema 1 ed il relativo corollario di M. Travo [19]), che il lemma sussite con $H^{s, r}\left(R^{n}\right)$ in luogo di $H^{s, r}\left(R_{+}^{n}\right)$.

Dalla definizione stessa di $H^{s, r}\left(R_{+}^{n}\right)$ si deduce evidentemente la tesi.

\section{2. - Valutazioni a priori per operatori a coefficienti costanti.}

Nel seguito di questo capitolo per comodità porremo $x=\left(x_{1}, \ldots, x_{n}\right)=$ $=(y, t)$ e $\xi=\left(\xi_{1}, \ldots, \xi_{n}\right)=(\eta, \tau)$, dove $y=\left(x_{1}, \ldots, x_{n-1}\right)$ e $\eta=\left(\xi_{1}, \ldots, \xi_{n-1}\right)$. 
M. Trorsi: Problemi al contorno con condizioni omogenee, ecc.

Assegnamo an operatore differenziale lineare a coefficienti costanti della forma

$$
A(D)=A\left(D_{y}, D_{t}\right)=\sum_{<\mu, q>=m} a_{\mu} D_{y}^{\mu^{\prime}} D_{t}^{\mu_{n}}
$$

sul quale faremo la seguente ipotesi:

$\left.\alpha_{1}\right) A(D)$ ə̀ quasi-elliltico, ciò̀ risulta

$$
A(\xi)=A(\eta, \tau) \neq 0 \quad \forall \xi=(\eta, \tau) \in R^{n}-\{0\}
$$

inoltre per ogni $\eta \in R^{n-1}-\{0\}$ il numero $\vee$ delle radici con parte immagina. ria positiva del polinomio, nella variabile complessa $z, A(\eta, z)$ è indipendente da $\eta\left({ }^{2}\right)$.

Indicheremo con $z_{1}^{+}(\eta), \ldots, z_{y}^{+}(\eta)$ le suddette radici e porremo

$$
M+(\eta, z)=\prod_{k=1}^{\nu}\left(z-z_{k}^{+}(\eta)\right)
$$

Assegnamo inoltre un sistema $\left\{B_{j}(D)\right\}_{j=1}^{v}$ di $\vee$ operatori differenziali lineari a coefficienti costanti della forma

$$
B_{j}(D)=B_{j}\left(D_{y}, D_{t}\right)=\sum_{<\mu, q>=p_{j}} b_{j \mu} D_{y}^{\mu^{\prime}} D_{t}^{\mu_{n}}, \quad 0 \leq p_{j} \leq m-q_{n},
$$

supponendo che sia verificata la seguente ipotesi:

$\alpha_{2}$ ) Gli operatori $B_{1}(D), \ldots, B_{y}(D)$ soddisfano la condizione complementare $\left(^{3}\right)$ rispetto ad $A(D)$, ciod per ogni $\eta \in R^{n-1}-\{0\}$ i polinomi, nella variabile complessa $z, B_{1}(\eta, z), \ldots, B_{v}(\eta, z)$ sono linearmente indipendenti modulo $M^{+}(\eta, z)$.

Sussistono i seguenti lemmi :

Lemma 2.1. - Se è verificata l'ipotesi $\left.\alpha_{1}\right)$, allora la $\left.\alpha_{2}\right)$ è condizione neces. saria e sufficiente affinchè, per ogni fissato $\eta \in R^{n-1}-\{0\}$, il problema

(2) Notoriamente, se $n>z$ l'operatore quasi-ellittico $A\left(D_{y}, D_{b}\right)$ soddisfa senz'altro a tale condizione.

(3) Cfr., ad es., la definizione 3.1 di T. Matsuzawa [21]. 


$$
\begin{aligned}
& A\left(\eta, D_{t}\right) \varphi(t)=0 \text { per } t>0, \\
& \left.B_{j}\left(\eta, D_{\imath}\right) \varphi(t)\right|_{t=0}=0, \quad j=1, \ldots, \nu,
\end{aligned}
$$

abbia in $H^{m}\left(R_{+}^{\mathrm{l}}\right)$ la sola soluzione nulla.

Lemma 2.2. - Se sono verificate le ipotesi $\alpha_{1}$ ) e $\alpha_{2}$ ), allora per ogni $\eta \in R^{n-1}$ $-\{0\}$ l'operatore

$$
\mathscr{S}_{\eta}: \varphi \rightarrow\left(A\left(\eta, D_{t}\right) \varphi,\left.B_{1}\left(\eta, D_{t}\right) \varphi\right|_{\imath=0}, \ldots,\left.B_{v}\left(\eta, D_{t}\right) \varphi\right|_{t=0}\right)
$$

risulta un isomorfismo (algebrico e topologico) $d i H^{m_{n}}\left(R_{+}^{1}\right)$ su $L^{2}\left(R_{+}^{1}\right) \times C^{\nu}\left({ }^{4}\right)$. Inoltre per ogni $\varphi \in H^{m_{n}}\left(R_{+}^{1}\right)$ si ha

$$
\|\varphi\|_{H^{m_{n_{(R}}}} \leq c(\eta)\left\|\mathfrak{S}_{\eta} \varphi\right\|_{L^{2}\left(R_{+}^{1}\right) \times C^{\nu}}
$$

dove $\eta \rightarrow c(\eta)$ è una funzione continua in $R^{n-1}-\{0\}$.

Le dimostrazioni di tali lemmi (ohe qui per brevità omettiamo) si ottengono con ragionamenti analoghi a quelli tenuti da J. L. Lions-E. Magenes (ofr. il n. 4 del cap. 2 di [20]) per dimostrare gli stessi risultati relativamente al caso ellittico, e ciò al caso in cui $m_{1}=\ldots=m_{n}=m$.

Osserviamo ora che l'operatore aggiunto $\mathfrak{S}_{\eta}^{*}$ di $\mathfrak{B}_{\eta}$ è l'operatore lineare $\mathrm{e}$

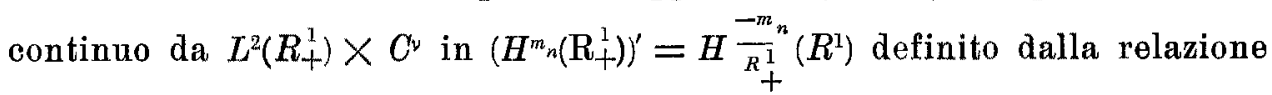

$$
<\varphi, \overline{\mathfrak{S}_{\eta}^{*} \Psi}>=\left\langle\mathfrak{S}_{\eta} \varphi, \bar{\Psi}>\right.
$$

per ogni $\varphi \in H^{m_{n}\left(R_{+}^{1}\right)}$ e per ogni $\Psi=\left(\psi, c_{1}, \ldots, c_{\nu}\right) \in L^{2}\left(R_{+}^{1}\right) \times C^{\nu}$.

Dal lemma 2.2 si deduce la maggiorazione

$$
\|\Psi\|_{L^{2}(R+) \times C^{\nu}}^{1} \leq c(\eta)\left\|\mathfrak{B}_{\eta}^{*} \Psi\right\|_{H}^{-i n_{n_{\left(R^{1}\right)}}} \quad \forall \Psi \in L^{2}\left(R_{+}^{1}\right) \times C^{\nu}
$$

Nel seguito, per ogni funzione $\psi \in L^{2}\left(R_{+}^{n}\right), n \geq 1$, indicheremo con $\psi_{0}$ il suo prolungamento a $L^{2}\left(R^{n}\right)$ con valori nulli per $t<0$, e per ogni distribuzione

(4) Indichiamo con $C$ lo spazio dei numeri complessi. 
$\varphi \in H^{s}\left(\boldsymbol{R}_{+}^{n}\right)$ indicheremo con $\varphi_{1}$ un suo assegnato prolungamento a $H^{s}\left(R^{n}\right)$.

Con tali notazioni dalla (2.6) si ha

$$
\begin{gathered}
<\varphi, \overline{\mathfrak{S}_{\eta}^{*} \Psi}>=<A\left(\eta, D_{t}\right) \varphi_{1}, \bar{\psi}_{0}>+\sum_{j=1}^{\nu}<\left.B_{j}\left(\eta, D_{t}\right) \varphi_{1}\right|_{t=0}, \overline{c_{j}}> \\
=<A\left(\eta, D_{t}\right) \varphi_{1}, \bar{\psi}_{0}>+\sum_{j=1}^{\nu}<B_{j}\left(\eta, D_{t}\right) \varphi_{1}, \overline{c_{j} \delta}> \\
=<\varphi_{1}, \overline{A^{*}\left(\eta, D_{t}\right) \psi_{0}}>+\sum_{j=1}^{\nu}<\varphi_{1}, \overline{B_{j}^{*}\left(\eta, D_{t}\right) c_{j} \delta}>
\end{gathered}
$$

dove $\delta$ denota la misura di Dirac nell'origine, $A^{*}\left(\eta, D_{t}\right)$ e $B_{j}^{*}\left(\eta, D_{t}\right)$ denotano gli operatori aggiunti formali rispettivamente di $A\left(\eta, D_{t}\right)$ e $B_{j}\left(\eta, D_{t}\right)$ (come operatori differenziali in $t$ ).

Se ne deduce che per ogni $\Psi=\left(\psi, c_{1}, \ldots, c_{\nu}\right) \in L^{2}\left(R_{+}^{1}\right) \times C^{\nu}$ risulta

$$
\mathfrak{S}_{\eta}^{*} \Psi=A^{*}\left(\eta, D_{t}\right) \psi_{0}(t)+\sum_{j=1}^{\nu} B_{j}^{*}\left(\eta, D_{t}\right) c_{j} \delta
$$

Consideriamo ora l'operatore

$$
\mathfrak{S}: u \rightarrow\left(A(D) u,\left.B_{1}(D) \boldsymbol{u}\right|_{t=0}, \ldots, B_{\imath}(D) u_{, t=0}\right)
$$

che, in conseguenza del lemma 1.7, risulta lineare e continuo da $H^{m}\left(R_{+}^{n}\right)$ in $L^{2}\left(R_{+}^{n}\right) \times \prod_{j=1}^{\nu} H^{m-p_{j}-q_{n} / 2}\left(R^{n-1}\right)$.

L'operatore aggiunto $\mathfrak{S}^{*}$ di $\mathfrak{S}$ è l'operatore lineare e continuo da $L^{2}\left(R_{+}^{n}\right)$ $\times \prod_{j=1}^{\nu} H^{-m+p_{j}+q_{n} / 2}\left(R^{n-1}\right)$ in $\left(H^{m}\left(R_{+}^{n}\right)\right)^{\prime}=H_{R_{+}^{n}}^{\frac{-m}{R^{n}}}\left(R^{n}\right)$ definito dalla relazione

$$
<u, \overline{\mathfrak{S}^{*} F}>=\langle\mathfrak{B} u, \bar{F}\rangle
$$

per ogni $u \in H^{m}\left(R_{+}^{n}\right)$ e per ogni $F=\left(f, g_{1}, \ldots, g_{\nu}\right) \in L^{2}\left(R_{+}^{n}\right) \times \prod_{j=1}^{\nu} H^{-m+p_{j}+q_{n} / 2}\left(R^{n-1}\right)$.

Denotati con $A^{*}(D)$ e $B_{j}^{*}(D)$ gli operatori aggiunti formali rispettivamente di $A(D)$ e $B_{j}(D)$, dalla $(2.10)$ si ha

$$
<u, \overline{\mathfrak{S}^{*} F}>=<A(D) u_{1}, \bar{f}_{0}>+\sum_{j=1}^{\nu}<\left.B_{j}(D) u_{1}\right|_{t=0}, \bar{g}_{j}>
$$




$$
\begin{gathered}
=\left\langle A(D) u_{1}, \bar{f}_{0}>+\sum_{j=1}^{\nu}\left\langle B_{j}(D) u_{1}, \overline{g_{j}(y) \otimes \delta(t)}>\right.\right. \\
=\left\langle u_{1}, \overline{A^{*}(D) f_{0}}>+\sum_{j=1}^{\nu}\left\langle u_{1}, \overline{B_{j}^{*}(D)\left(g_{j}(y) \otimes \delta(t)\right)}>.\right.\right.
\end{gathered}
$$
risulta

Se ne deduce che per ogni $F=\left(f, g_{1}, \ldots, g_{\nu}\right) \in L^{2}\left(R_{+}^{n}\right) \times \prod_{j=1}^{y} H^{-m+p_{j}+q_{n} / 2}\left(R^{n-1}\right)$

$$
\mathfrak{S}^{*} F=A^{*}(D) f_{0}+\sum_{j=1}^{v} B_{j}^{*}(D)\left(g_{j}(y) \otimes \delta(t)\right)
$$

Vogliamo dimostrare il seguente teorema che estende al caso quasi-ellittico la formula (4 27) del cap. 2 di J. L. Lions-E. Magenes [20]:

Teonema 2.1. - Se sono verificate le ipotesi $\left.\alpha_{1}\right)$ e $\left.\alpha_{2}\right)$, per ogni $F=\left(f, g_{1}\right.$, $\left.\ldots, g_{v}\right) \in L^{2}\left(R_{+}^{n}\right) \times \prod_{j=1}^{\nu} H^{-m+p_{j}+q_{n} / 2}\left(R^{n-1}\right)$ sussiste la limitazione

$$
\begin{aligned}
& \|F\|_{L^{2}\left(R_{+}^{n}\right) \times \prod_{j=1}^{\nu} H^{-m+p_{j}+\left.q_{n}\right|^{2}\left(R^{n-1}\right)} \leq c\left(\left\|\mathfrak{S}^{*} F\right\|_{H}-\pi^{n}\left(R^{n}\right)\right.} \\
& \left.+\left\|F_{0}\right\|_{H^{-1}}\left(R^{n}\right) \times \prod_{j=1}^{v} H^{-m+p_{j}-q_{n} / 2\left(R^{n-1}\right)}\right),
\end{aligned}
$$

dove $F_{0}=\left(f_{0}, g_{1}, \ldots, g_{y}\right)$ e dove $c \dot{e}$ una costante indipendente da $F$.

Infatti ( $\left.{ }^{5}\right)$, dalle $(2.7)$ e $(2.8)$ si ha per $\Psi=\left(\psi, c_{1}, \ldots, c_{y}\right) \in L^{2}\left(R_{+}^{1}\right) \times C^{\nu}$ e per $\langle\eta\rangle=1$

$$
\begin{aligned}
\int_{0}^{+\infty}|\psi(t)|^{2} d t+\sum_{j=1}^{\nu}\left|c_{j}\right|^{2} & \leq k_{0} \int_{-\infty}^{+\infty}\left(1+\tau^{2}\right)^{-m_{n}} \mid A^{*}(\eta, \tau) \int_{0}^{+\infty} e^{-i \tau t} \psi(t) d t \\
& +\left.\sum_{j=1}^{\nu} B_{j}^{*}(\eta, \tau) c_{j}\right|^{2} d \tau
\end{aligned}
$$

dove $k_{0}$ ̀े una costante indipendente da $\eta$ e $\Psi$.

(5) Otterremo la dimostrazione di questo teorema con ragionamenti concettualmente analoghi a quelli tenuti da J.L. Lrons e E. MAGExes per dimostrare l'analogo risultato relativo al caso ellitico (efr. la dimostrazione della citata formula (4.27) del cap. 2 di [20]). 
Osserviamo che posto, per ogni $\eta \in R^{n-1}-\{0\}, \eta^{\prime}=\left(\langle\eta\rangle-q_{1} \eta_{1}, \ldots\right.$,

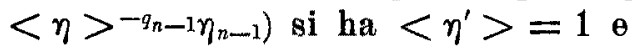

$$
\begin{aligned}
& A^{*}\left(\eta^{\prime}, \tau\right)=\langle\eta\rangle^{-m} A^{*}\left(\eta,\left\langle\eta>^{q_{n} \tau}\right),\right.
\end{aligned}
$$

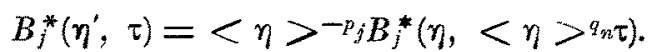

Se ne deduce che per ogni $\eta \in R^{n-1}-\{0\}$ si ha

$$
\begin{gathered}
\int_{0}^{+\infty}\left|\psi\left(<\eta>^{q_{n}} t\right)\right|^{2} d t+<\eta>^{-q_{n}} \sum_{j=1}^{\nu}\left|c_{j}\right|^{2} \\
\leq k_{0} \int_{-\infty}^{+\infty}\left(<\eta>^{2 q_{n}}+\tau^{2}\right)^{-m_{n}} \mid A^{*}(\eta, \tau) \int_{0}^{+\infty} e^{-i \tau t} \psi\left(<\eta>^{q_{n}} t\right) d t \\
+\sum_{j=1}^{\nu} B_{j}^{*}(\eta, \tau) c_{j}<\eta>\left.^{m-p_{j}-q_{n}}\right|^{2} d \tau .
\end{gathered}
$$

Assegnato ora $F=\left(f, g_{1}, \ldots, g_{v}\right) \in L^{2}\left(R_{+}^{n}\right) \times \prod_{j=1}^{\nu} H^{-m+p_{j}+q_{m} / 2}\left(R^{n-1}\right)$, sostituiamo nella (2.13) $\bar{f}(\eta, t)$ a $\psi\left(\left\langle\eta>^{\left.q_{n} t\right)}\right.\right.$ e $\bar{g}_{j}(\eta)$ a $c_{j}<\eta>^{m-p_{j}-q_{n}}$. Tenuto allora conto della (2.11) si ha per ogni $\eta \in R^{n-1}-\{0\}$

$$
\begin{gathered}
\int_{0}^{+\infty}|\bar{f}(\eta, t)|^{2} d t+\sum_{j=1}^{v}<\eta>^{2\left(-m+p_{j}+q_{n} / 2\right)}\left|\widehat{g_{j}}(\eta)\right|^{2} \\
\leq k_{0} \int_{-\infty}^{+\infty}\left(<\eta>^{2 q_{n}}+\tau^{2}\right)^{-m_{n}}\left|\widehat{\mathfrak{S}^{*} F^{2}}\right|^{2} d \tau .
\end{gathered}
$$

Nel seguito di questa dimostrazione denoteremo con $k_{1}, \ldots, k_{5}$ delle costanti indipendenti da $F$.

Dalla (2.14) si deduce la limitazione

$$
\begin{gathered}
\int_{<\eta>\geq 1} d \eta \int_{0}^{+\infty}|\widehat{f}(\eta, t)|^{2} d t+\sum_{j=1}^{\nu} \int_{<\eta>\geq 1}\left(1+<\eta>^{2}\right)^{-m+p_{j}+\left.q_{n}\right|^{2}}\left|\widehat{g_{j}}(\eta)\right|^{2} d \eta \\
\leq k_{1}\left\|\mathfrak{S}^{*} F\right\|^{2}{ }^{2}-m_{\left(R^{n}\right)} .
\end{gathered}
$$


346

M. Trorsi: Problemi al contorno con condizioni omogenee, ecc.

Inoltre si ha evidentemente

$$
\begin{gathered}
\int_{<\eta>\leq 1}\left(1+<\eta>^{2}\right)^{-m+p_{j}+q_{n} / 2}\left|\widehat{g}_{j}(\eta)\right|^{2} d \eta \\
\leq k_{2}\left\|g_{j}\right\|^{2}{ }^{-m+p_{j}-q_{n} /{ }^{2}}\left(R^{n-1}\right),
\end{gathered}
$$

mentre con le stesse considerazioni tenute in [20] per stabilire la (4.33) del cap. 2 , si dimostra che risulta

$$
\begin{gathered}
\int_{<n>\leq 1} d \eta \int_{0}|\widehat{f}(\eta, t)|^{2} d t \leq k_{3}\left(\left\|f_{0}\right\|_{H^{2}-{ }^{2}\left(R^{n}\right)}\right. \\
\left.+\int_{<\eta>\leq 1}\left\|\frac{\partial^{m} \widehat{f_{0}}(\eta, t)}{\partial t^{m_{n}}}\right\|_{H^{-m_{n}\left(R^{1}\right)}}^{2} d \eta\right) .
\end{gathered}
$$

Per maggiorare l'integrale a secondo membro della (2.17), osserviamo che si ha (si tenga presente la (2.11))

$$
\begin{aligned}
& a \tau^{m_{n}} \tilde{f}_{0}(\eta, \tau)=A^{*}(\eta, \tau) \tilde{f}_{0}(\eta, \tau)-\sum_{k=0}^{m_{n}-1} a_{k}(\eta) \tau^{k} \tilde{f}_{0}(\eta, \tau) \\
& =\widetilde{\mathcal{G}^{*} F^{\prime}}-\sum_{k=0}^{m_{n}-1} a_{k}(\eta) \tau^{k} \tilde{f}_{0}(\eta, \tau)-\sum_{j=1}^{v} B_{j}^{*}(\eta, \tau) \tilde{g}_{j}(\eta),
\end{aligned}
$$

dove a d una costante $\neq 0$ e le $a_{k}(\eta)$ sono delle funzioni continue di $\eta$. Se ne deduce che risulta

$$
\begin{aligned}
& \int_{<\eta>\leq 1}\left\|\frac{\partial^{m_{n}} \widehat{\widehat{f_{0}}(\eta, t)}}{\partial t^{m}}\right\|_{H^{-m_{n}}}^{2} d \eta=\int_{<\eta>\leq 1} d \eta \int_{-\infty}^{+\infty}\left(1+\tau^{2}\right)^{-m_{n}}\left|\tau^{m_{n}} \tilde{f}_{0}(\eta, \tau)\right|^{2} d \tau \\
& \leq k_{4}\left(\int_{<n>\leq 1} d \eta \int_{-\infty}^{+\infty}\left(1+\tau^{2}\right)^{-m_{n}}\left|\widehat{\mathfrak{S}^{*} F}\right|^{2} d \tau\right. \\
& \left.+\int_{<\eta>\leq 1} d \eta \int_{-\infty}^{+\infty}\left(1+\tau^{2}\right)^{-1}\left|\tilde{f}_{0}(\eta, \tau)\right|^{2} d \tau+\sum_{j=1}^{\nu} \int_{\langle\eta>\leq 1}\left|\bar{g}_{j}(\eta)\right|^{2} d \eta\right)
\end{aligned}
$$




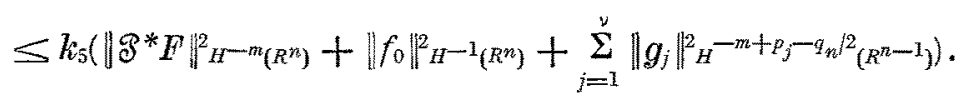

Dalle $(2.15)-(2.18)$ si deduce la (2.12), e quindi si ha la tesi.

\section{3. - Valatazioni a priori per operatori a coefficienti variabili.}

Assegnamo ora un operatore differenziale lineare della forma

$$
A(x, D)=\sum_{<\mu, q>\leq m} a_{\mu}(x) D^{\mu},
$$

a coefficienti $a_{\mu}(x) \in C_{0}^{\infty}\left(R^{n}\right)$ e con la seguente condizione:

$\beta_{1}$ ) L'operatore a coefficienti costanti

$$
A_{0}(D)=A_{0}(0, D)=\sum_{\langle\mu, q>=m} a_{\mu}(0) D^{\mu}
$$

verifica l'ipotesi $\alpha_{1}$ ).

Assegnamo inoltre un sistema $\left\{\left.B_{j}(y, D)\right|_{j=1} ^{\psi}\right.$ di $\vee$ operatori differenziali lineari della forma

$$
B_{j}(y, D)=\sum_{<\mu, q>\leq p_{j}} b_{j \mu}(y) D^{\mu}, \quad 0 \leq p_{j} \leq m-q_{n},
$$

a coefficienti $b_{j \mu}(y) \in C_{0}^{\infty}\left(R^{n-1}\right)$ e con la seguente condizione:

$\beta_{2}$ ) Gli operatori a coefficienti costanti

$$
B_{0 j}(D)=B_{0 j}(0, D)=\sum_{<\mu, q>=p_{j}} b_{j \mu}(0) D^{\mu}, \quad j=1, \ldots, \quad,
$$

verificano la condizione complementare rispetto all'operatore $A_{0}(0, D)$.

Chiameremo parte principale di $A(x, D)$, l'operatore

$$
A_{0}(x, D)=\sum_{<\mu, q>=m} a_{\mu \mu}(x) D^{\mu}
$$

e parte principale di $B_{j}(y, D)$, l'operatore

$$
B_{j 0}(y, D)=\sum_{<\mu, q>=p_{j}}^{\Sigma} b_{j k}(y) D^{\mu} .
$$


Osserviamo che, in conseguenza del lemma 1.7, l'operatore

$$
P: u \rightarrow\left(A(x, D) u,\left.B_{1}(y, D) u\right|_{t=0}, \ldots,\left.B_{\vee}(y, D) u\right|_{t=0}\right)
$$

risulta, per ogni $r$ reale, lineare $\theta$ continuo da $H^{m_{r} r}\left(R_{+}^{n}\right)$ in $H^{0, r}\left(R_{+}^{n}\right) \times$ $\times \prod_{j=1}^{\stackrel{n}{n}} H^{m+r-p_{j}-q_{n} / 2}\left(R^{n-1}\right)$.

L'operatore aggiunto $P^{*}$ di $P$ risulta allora lineare e continuo da $H^{0_{0}-r}\left(R_{+}^{n}\right) \times \prod_{j=1}^{\forall} H^{-m-r+p_{j}+q_{x} / 2}\left(R^{n-1}\right)$ in $\left(H^{m, r}\left(R_{+}^{n}\right)\right)^{\prime}=H_{R_{+}^{n}}^{-m_{,}-r}\left(R^{n}\right)$ e, con le stesse considerazioni tenute per stabilire la (2.11), si prova che per ogni $F=(t$, $\left.g_{1}, \ldots, g_{v}\right) \in H^{0,-r}\left(R_{+}^{n}\right) \times \prod_{j=1}^{v} H^{-m-r+p_{j}+q_{n} / 2}\left(R^{n-1}\right)$ si ha

$$
P^{*} F=A^{*}(x, D) f_{0}(x)+\sum_{j=1}^{v} B_{j}^{*}(y, D)\left(g_{j}(y) \otimes \delta(t)\right)
$$

dove $A^{*}(x, D)$ e $B_{j}^{*}(y, D)$ denotano gli operatori aggiunti formali rispettivamente di $A(x, D)$ e $B_{j}(y, D)$.

Scriveremo $A^{*}$ e $B_{j}^{*}$ nella forma

$$
\begin{gathered}
A^{*}(x, D)=\sum_{<\mu, q>\leq m} a_{\mu}^{*}(x) D^{\mu}, \\
B_{j}^{*}(y, D)=\sum_{<\mu, q>\leq p_{j}} b_{j \mu}^{*}(y) D^{\mu}, \quad j=1, \ldots, \quad \text {. } .
\end{gathered}
$$

Per ogni numero reale $\rho>0$ porremo

$$
\begin{gathered}
\Gamma(\rho)=\left\{x \in R^{n}|| x \mid<\rho\right\}, \\
\Sigma(\rho)=\left\{x \in R^{n}|| x \mid<\rho, x_{n} \geq 0\right\}, \\
\sigma(\rho)=\left\{y \in R^{n-1}|| y \mid<\rho\right\} .
\end{gathered}
$$

Vogliamo dimostrare il seguente

Teorema 3.1. - Se sono verificate le ipotesi $\left.\beta_{1}\right)$ e $\beta_{2}$ ), esiste un numero reale $\rho_{1}>0$ tale che per ogni $F=\left(f, g_{1}, \ldots, g_{v}\right) \in H^{0, r}\left(R_{+}^{n}\right) \times \prod_{j=1}^{\nu} H^{-m+r+p_{j}+q_{n} l^{2}}\left(R^{n-1}\right)$, 
$r$ reale, con $f$ e $g_{j}$ aventi supporti contenuti in $\Sigma\left(\rho_{1}\right)$ e $\sigma\left(\rho_{1}\right)$ rispettivamente, sussiste la limitazione

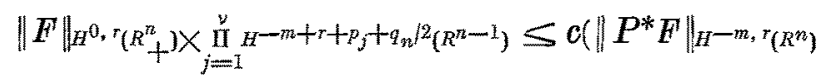

$$
\begin{aligned}
& \left.+\|F\|_{H^{0}, r-1}\left(R_{+}^{n}\right) \times \prod_{j=1}^{\nu}{ }^{H-m+r-1+p_{j}}+q_{n} / 2^{2}\left(R^{n-1}\right)\right),
\end{aligned}
$$

dove c è una costante indipendente da $F$ e dove, se $r=0$, si può sostituire la norma di f in $H^{0,-1}\left(R_{+}^{n}\right)$ con la norma di fo in $H^{-1}\left(R^{n}\right)$.

Infatti, indichiamo con $A_{0}^{*}(x, D)$ e $B_{0 j}^{*}(y, D)$ le parti principali di $A^{*}(x, D)$ e $B_{j}^{*}(y, D)$.

Evidentemente, $A_{0}^{*}(0, D)$ e $B_{0}^{*}(0, D)$ sono gli operatori aggiunti formali di $A_{0}(0, D)$ e $B_{0 j}(0, D)$.

Introduciamo la distribuzione $\lambda_{r}(y) s u R^{n-1}$ tale che

$$
\bar{\lambda}_{r}(\eta)=\left(1+\left\langle\eta>^{2}\right)^{r / 2}\right.
$$

ed osserviamo che per ogni $F=\left(f, g_{1}, \ldots, g_{\nu}\right) \in H^{0, r}\left(R_{+}^{n}\right) \times \prod_{j=1}^{\nu} H^{-m+r+p_{j}+q_{n} / 2}\left(R^{n-1}\right)$ risulta $\lambda_{r} * F=\left(\lambda_{r} * f, \lambda_{r} * g_{1}, \ldots, \lambda_{r} * g_{\nu}\right) \in L^{2}\left(R_{+}^{n}\right) \times \prod_{j=1}^{v} H^{-m+p_{j}+q_{n} / 2}\left(R^{n-1}\right)$ e si ha

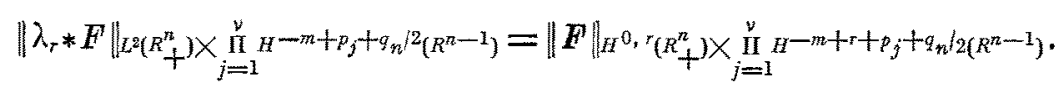

Assegnato allora un vettore $F$ della suddetta classe, dal teorema 2.1 si deduce che si ha

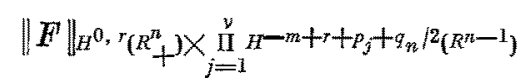

$$
\begin{aligned}
& \leq c_{1}\left(\left\|A_{0}^{*}(0, D) f_{0}+\sum_{j=1}^{\nu} B_{0 j}^{*}(0, D)\left(g_{j}(y) \otimes \delta(t)\right)\right\|_{H-m, r_{\left(R^{n}\right)}}\right. \\
& \left.+\left\|F_{0}\right\|_{H}-1, r_{\left(R^{n}\right)}\right) \underset{j=1}{\left.\stackrel{y}{H} H^{-m+r}+p_{j}-q_{n} / 2_{\left(R^{n-1}\right)}\right)}
\end{aligned}
$$

qui e nel seguito di questa dimostrazione denotiamo con $c_{1}, c_{2}, \theta c_{3}$ delle costanti positive indipendenti da $F$. 
Dalla (3.11) consegue la limitazione

$$
\begin{aligned}
& \left.\left\|F^{\prime}\right\|_{H^{0}}, r_{\left(R_{+}^{n}\right.}^{n}\right) \times \prod_{j=1}{ }^{\left.H^{-m}+r+p_{j}+q_{n}\right)^{2}\left(R^{n-1}\right)} \leq c_{2}\left(\left\|P^{*} F\right\|_{H-m, r_{\left(R^{n}\right)}}\right. \\
& \left.+\left\|F_{0}\right\|_{H-1, r_{\left(R^{n}\right)}} \prod_{j=1}^{\prod_{H}{ }^{\prime}-m+r+p_{j}-q_{n} / 2\left(R^{n-1}\right)}+I_{1}(F)+I_{2}(F)\right),
\end{aligned}
$$

dove si è posto

$$
\begin{gathered}
I_{1}(F)=\|\left(A_{0}^{*}(0, D)-A_{0}^{*}(x, D)\right) f_{0}+\sum_{j=1}^{v}\left(B_{0 j}^{*}(0, D)-B_{0 j}^{*}(y, D)\left(g_{j}(y) \otimes \delta(t)\right) \|_{H}-m, r\left(R_{n}\right),\right. \\
I_{2}\left(F^{\prime}\right)=\sum_{<\mu, q><m}\left\|D^{\mu} f_{0}\right\|_{H}-m, r_{\left(R^{n}\right)}+\sum_{j=1}^{v} \sum_{<\mu, q><p_{j}}^{\Sigma}\left\|D^{\mu}\left(g_{j}(y) \otimes \delta(t)\right)\right\|_{H}-m, r_{\left(R^{n}\right)} .
\end{gathered}
$$

In conseguenza del lemma 1.3, si ha che per ogni $\varepsilon>0$ esiste un numero reale $\rho>0$ tale che, se $f$ e $g_{j}$ hanno supporti contenuti rispettivamente in $\Sigma(\rho)$ e $\sigma(\rho)$, risulta

$$
\begin{gathered}
I_{1}(F) \leq \varepsilon\left(\left\|f_{0}\right\|_{H^{0, r}\left(R^{n}\right)}+\sum_{j=1}^{\nu}\left\|g_{j}(y) \otimes \delta(t)\right\|_{H-m+p_{j}, r}\left(R^{n_{3}}\right)\right) \\
+c(\varepsilon)\left(\left\|f_{0}\right\|_{H^{0, r-1}\left(R^{n}\right)}+\sum_{j=1}^{\nu}\left\|g_{j}(y) \otimes \delta(t)\right\|_{H}-m+p_{j}, r-{ }^{-1}\left(R^{n}\right)\right)
\end{gathered}
$$

dove, se $r=0$, si può sostituire la morma di $f_{0}$ in $H^{0,-1}\left(R^{n}\right)$ con la norma di $f_{0}$ in $H^{-1}\left(R^{n}\right)$.

Inoltre, in conseguenza del lemma 1.2, si ha per ogni $\varepsilon>0$

$$
\begin{aligned}
& I_{2}(F) \leq \varepsilon\left(\left\|f_{0}\right\|_{H^{0,}, r_{\left(R^{n}\right)}}+\sum_{j=1}^{\nu}\left\|g_{j}(y) \otimes \delta(t)\right\|_{\left.H-m+p_{j}, r_{\left(R^{n}\right)}\right)}\right. \\
& +c_{1}(\varepsilon)\left(\left\|f_{0}\right\|_{H^{-1}, r_{\left(R^{n}\right)}}+\sum_{j=1}^{\nu}\left\|g_{j}(y) \otimes \delta(t)\right\|_{\left.H-m+p_{j}-1, r_{(} R^{n}\right)}\right)
\end{aligned}
$$

D'altra parte si verifica facilmente che, comunque si assegnano i numeri reali $l>q_{n} / 2$ e $k$, risulta

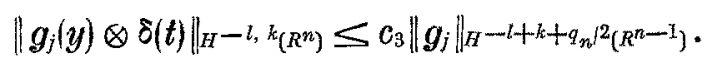

Dalle (3.12) - (3.15) si deduce in modo ovvio la tesi. 


\section{4. - Regolarizzazione parziale alla frontiera.}

In questo numero indicheremo con $\omega(y)$ un'assegnata funzione reale e non negativa di classe $C_{0}^{\infty}\left(R^{n-1}\right)$ e tale che

$$
\int_{R^{n-1}} \omega(y) d y=1, \quad \operatorname{supp} \omega(y)=\{y|| y \mid \leq 1\}
$$

Porremo per ogni $\varepsilon>0$ e per ogni distribuzione $u(y, t)$ :

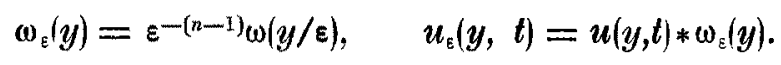

Da noti risultati (cfr., ad es., il cap. I di L. Hörmander [18]) segue che se $u \in H^{s, r}\left(R_{+}^{n}\right)$ [risp. $u \in H^{r}\left(R^{n-1}\right)$ ] allora $u_{\varepsilon} \in H^{s, k}\left(R_{+}^{n}\right)$ [risp. $u_{\varepsilon} \in H^{k}\left(R^{n-1}\right)$ ] per ogni $k$ reale; inoltre riesce

$$
\lim _{\varepsilon \rightarrow 0}\left\|u_{\varepsilon}-u\right\|_{H^{s, r}\left(R_{+}^{n}\right)}=0 \text { [risp. } \lim _{\varepsilon \rightarrow 0}\left\|u_{\varepsilon}-u\right\|_{H^{r^{r}}\left(R^{n-1}\right)}=0 \text { ]. }
$$

Per ogni funzione $\zeta(y, t)$ definita in $R^{n}$ e per ogni vettore $F=(f(y, t)$, $\left.g_{1}(y), \ldots, g_{v}(y)\right)$ porremo

$$
\begin{gathered}
\dot{\zeta}(y)=\zeta(y, 0), \quad \zeta F=\left(\zeta t, \dot{\zeta} g_{1}, \ldots, \dot{\zeta} g_{v}\right) \\
(\zeta F)_{\varepsilon}=\left((\zeta)_{\varepsilon},\left(\zeta g_{1}\right)_{\varepsilon}, \ldots,\left(\zeta g_{y}\right)_{\varepsilon}\right) .
\end{gathered}
$$

Vogliamo dimostrare il seguente

Teorema 4.1. - Siano verificate le ipotesi $\left.\left.\beta_{1}\right), \beta_{2}\right)$ e sia $F=\left(f, g_{1}, \ldots, g_{v}\right)$ un assegnato vettore di classe $L^{2}\left(R_{+}^{n}\right) \times\left.\prod_{j=1}^{\nu} H^{-m+p_{j}+q_{n}}\right|^{2}\left(R^{n-1}\right)$.

Esiste, per ogni intero $k>0$, un numero positivo $\rho_{0} \leq \rho_{1}$ (dove $\rho_{1}$ è il numero definito dal teorema 3.1) tale che se risulta, per ogni $\zeta \in C_{0}^{\infty}\left(R^{n}\right)$ e con supporto contenuto in $\Gamma(\rho)$ per un $\rho<\rho_{0}$,

$$
\zeta P^{*} F \in H^{-m, k}\left(R^{n}\right)
$$


allora si ha

$$
\zeta F \in H^{0, k}\left(R_{+}^{n}\right) \times \prod_{j=1}^{\nu} H^{-m+k+p_{j}+q_{n} / 2}\left(R^{n-1}\right) .
$$

Ragionando per ricorrenza, ei basterà stabilire la (4.3) nell'ipotesi che sussista la (4.2) e che si abbia

$$
\zeta F^{\prime} \in H^{0, k-1}\left(R_{+}^{n}\right) \times \prod_{j=1}^{\nu} H^{-m+k-1+p_{j}+\left.q_{n}\right|^{2}}\left(R^{n-1}\right) .
$$

In ciò faremo in parte uso della tecnica utilizzata da T. Matsuzawa per dimostrare il teorema 8.1 di [21].

Fissati $\rho<\rho_{0}, \zeta \in C_{0}^{\infty}\left(R^{n}\right)$ ed a supporto contenuto in $\Gamma(\rho)$, consideriamo il vettore $(\zeta F)_{\text {s }}$ per $\varepsilon<\left(\rho_{0}-\rho\right) / 2$.

Le funzioni $(\zeta f)_{\varepsilon}$ e $\left(\dot{\zeta} g_{j}\right)_{\varepsilon}$ hanno supporti contenuti rispettivamente in $\Sigma\left(\rho^{\prime}\right)$ e $\sigma\left(\rho^{\prime}\right)$, dove $\rho^{\prime}=\left(\rho+\rho_{0}\right) / 2$, e quindi per il teorema 3.1 si ha la limitazione

$$
\begin{aligned}
& \left.\left\|(\zeta F)_{\varepsilon}\right\|_{H^{0}, k_{(}} R_{+}^{n}\right) \times \prod_{j=1}^{\mathscr{n}} H^{-m+k+p_{j}+\left.q_{n}\right|^{2}\left(R^{n-1}\right)} \leq c_{1}\left(\left\|P^{*}(\zeta F)_{\varepsilon}\right\|_{H}-m, k_{\left(R^{n}\right)}\right. \\
& \left.+\left\|(\zeta F)_{\varepsilon}\right\|_{H^{0, k-1}}\left(R_{+}^{n}\right) \times \prod_{j=1}^{h} H^{-m+k-1}+p_{j}+q_{n} /{ }^{2}\left(R^{n-1}\right)\right) ;
\end{aligned}
$$

nel corso di questa dimostrazione con $c_{1}, \ldots, c_{6}$ indichiamo delle costanti positive indipendenti da $\varepsilon$.

Osserviamo ora che risulta

$$
\begin{aligned}
& P^{*}(\zeta F)=\zeta P^{*} F+\sum_{<\mu, q>\leq m} \sum_{\alpha<\mu}\left(\begin{array}{c}
\mu \\
\alpha
\end{array}\right) a_{\mu}^{*} D^{\mu-\alpha \zeta} \zeta D^{\alpha} f_{0}
\end{aligned}
$$

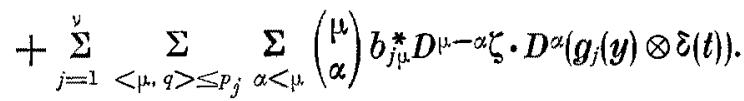

Si verifica facilmente, in conseguenza dell'ipotesi di induzione (4.4) $\theta$ della (3.15), che, per ogni funzione $\psi \in C_{0}^{\infty}\left(R^{n}\right)$ ed a supporto contenuto in una sfera con centro nell'origine e di raggio $<\rho_{0}$, risulta

$$
\begin{aligned}
D^{\alpha}\left(\psi f_{0}\right) \in H^{-m, k}\left(R^{n}\right) & \text { per } \alpha<\mu, \text { dove }<\mu, q>\leq m, \\
D^{\alpha}\left(\left(\dot{\psi} g_{j}\right) \otimes \delta\right) \in H^{-m, k}\left(R^{n}\right) & \text { per } \alpha<\mu, \text { dove }<\mu, q>\leq p_{j} .
\end{aligned}
$$


Le (4.2), (4.6), (4.7) e (4.8) implicano che si ha

$$
P^{*}(\zeta F) \in H^{-m, k}\left(R^{n}\right)
$$

Inoltre, con ragionamenti analoghi a quelli tenuti da L. Hörmander per stabilire la $(2.4 .18)$ di [18], si prova che si ha

$$
\left\|a_{\mu}^{*}(\zeta f)_{\varepsilon}-\left(a_{\mu}^{*} \zeta f\right)_{\varepsilon}\right\|_{H^{0, k_{(}}\left(R_{+}^{n}\right)} \leq c_{2}\|\zeta f\|_{R^{0, k-1}\left(R_{+}^{n}\right)}
$$

e (si tenga presente la (3.15))

$$
\begin{aligned}
& \left\|b_{j \mu}^{*}\left(\left(\dot{\zeta} g_{j}\right) \otimes \delta\right)_{\varepsilon}-\left(b_{j \mu}^{*}\left(\left(\dot{\zeta} g_{j}\right) \otimes \delta\right)\right)_{\varepsilon}\right\|_{k-m+p_{j}, k_{\left(R^{n}\right)}} \\
& \leq c_{3}\left\|b_{j \mu}^{*}\left(\dot{\zeta} g_{j}\right)_{\varepsilon}-\left(b_{j \mu}^{*} \dot{\zeta} g_{j}\right)_{\varepsilon}\right\| H^{-m++k+p_{j}+q_{n} I^{2}\left(R^{n-1}\right)} \\
& \leq c_{4}\left\|\zeta g_{j}\right\|_{H-m+k-1+p_{j}+q_{n} / 2\left(R^{n-1}\right)} .
\end{aligned}
$$

Dalle (4.4), (4.10) e (4.11) segue che si ha

$$
\begin{gathered}
\| P^{*}(\zeta F)_{\varepsilon}-\left(P^{*}(\zeta F)_{\varepsilon} \|_{H}-m, k_{(R)} R^{n}\right) \leq c_{5}(1 \\
+\sum_{<\mu, q>\leq_{m}} \sum_{\alpha<\mu}\left\|D^{\mu-\alpha} a_{\mu}^{*} \cdot D^{\alpha}\left(\zeta f_{0}\right)_{\varepsilon}-\left(D^{\mu-\alpha} a_{\mu}^{*} \cdot D^{\alpha}\left(\zeta f_{0}\right)\right)_{\varepsilon}\right\|_{H}-m, k_{\left(R^{n}\right)} \\
+\sum_{j=1}^{\nu} \sum_{<\mu, q>\leq p_{j}}^{\sum} \sum_{\alpha<\mu} \| D^{\mu-\alpha} b_{j \mu}^{*} \cdot D^{\alpha}\left(\left(\zeta g_{j}\right)_{\varepsilon} \otimes \delta\right)-\left(D^{\mu-\alpha} b_{j \mu}^{*} \cdot D^{\alpha}\left(\left(\zeta g_{j}\right)^{\otimes} \otimes \delta\right)_{\varepsilon} \|_{H}-m, k_{\left(R^{n}\right)}\right) .
\end{gathered}
$$

D'altra parte si può scrivere

$$
\begin{gathered}
\left\|P^{*}(\zeta F)_{\varepsilon}\right\|_{H}-m, k_{\left(R^{n}\right)} \leq\left\|\left(P^{*}(\zeta F)\right)_{\varepsilon}\right\|_{H-m, k_{\left(R^{n}\right)}} \\
+\left\|P^{*}(\zeta F)_{\varepsilon}-\left(P^{*}\left(\zeta F^{\prime}\right)\right)_{\varepsilon}\right\|_{H-m, k^{k}\left(R^{n}\right)} .
\end{gathered}
$$

Dalle relazioni precedenti e dalla (4.1) si deduce che esiste un numero $\varepsilon_{0}>0$ tale che per ogni numero positivo $\varepsilon \leq \varepsilon_{0}$ si ha

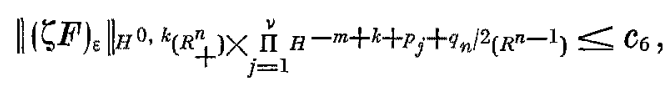

e tale relazione implica, per il teorema di BANACH-SAKs, la (4.3). Si ha cosi la tesi. 


\section{5. - Regolarizzazione alla frontiera.}

Consideriamo un operatore differenziale lineare $A(x, D)$ della forma (3.1) ed a coefficienti $a_{\mu}(x)$ di classe $C^{\infty}\left(\overline{R_{+}^{n}}\right)$.

Sussiste il seguente teorema, dove indichiamo con $\mathfrak{H}^{s}\left(R_{+}^{n}\right), s$ reale, 10 spazio a cui si riduce $H^{s}\left(R_{+}^{n}\right)$ quando $m_{1}=\ldots=m_{n}=2$.

TeOrema 5.1. - Siano $k$ un intero $>0$ e po un numero reale $>0$.

Se riesce $a(x)=a_{\left(0, \ldots, 0, m_{n}\right)}(x) \neq 0$ in $\Sigma\left(\rho_{0}\right)$, allora per ogni distribuzione f su $R_{+}^{n}$ tale che

$$
\begin{array}{cc}
\zeta A f \in \mathcal{H}^{-m+\left(m-m_{n}+1\right) k}\left(R_{+}^{n}\right) & \forall \zeta \in C_{0}^{\infty}\left(\overline{R_{+}^{n}}\right) \text { con supp } \zeta \subset \Sigma\left(\rho_{0}\right), \\
D_{y}^{\tau} f \in L^{2}\left(\Sigma\left(\rho_{0}\right)\right), & |\tau| \leq\left(m-m_{n}+1\right) k,
\end{array}
$$

si ha per $\rho<p_{0}$

$$
D_{y}^{\tau} D_{l}^{l} f \in L^{2}(\Sigma(\rho)), \quad|\tau| \leq\left(m-m_{n}+1\right)(k-l), \quad l \leq k .
$$

Supponiamo che la (5.3) sia vera per $l=r$, dove $r \leq k-1$, e dimostriamo che di conseguenza essa è vera per $l=r+1$.

Evidentemente, possiamo limitarci a dimostrare che

$$
D_{i}^{r+\mathrm{x}} f \in L^{2}(\Sigma(\rho))
$$

e che se si ha, per un generico intero positivo $h \leq\left(m-m_{n}+1\right)(k-r-1)$,

$$
D_{y}^{\tau} D_{t}^{r+1} f \in L^{2}(\Sigma(\rho)), \quad|\tau| \leq h-1
$$

allora si ha anche

$$
D_{y}^{\tau} D_{t}^{r+1} f \in L^{2}(\Sigma(\rho)), \quad|\tau|=h .
$$

Otterremo tale dimostrazione facendo uso di una tecnica che generalizza un noto procedimento relativo al caso ellittico (cfr., ad es., la dimostrazione del lemma 9.5 di S. AgMon [2]). 
Osserviamo che, comunque si assegna una $n$-pla di interi non negativi $\alpha$, si può scrivere

$$
\begin{aligned}
& a D_{t}^{m_{n}} D^{\alpha} f=D^{\alpha} A f-\sum_{\substack{<, q, q \leq m m \\
\mu_{n}<m_{n}}} \sum_{\beta \leq \alpha}\left(\begin{array}{l}
\alpha \\
\beta
\end{array}\right) D^{\alpha-\beta} a_{\mu} \cdot D^{\mu} D^{\beta} f \\
&-\sum_{\beta<\alpha}\left(\begin{array}{l}
\alpha \\
\beta
\end{array}\right) D^{\alpha-\beta} \alpha \cdot D_{t}^{m_{n}} D^{\beta} f .
\end{aligned}
$$

Ne segue che per ogni funzione $\varphi \in C_{0}^{30}\left(R_{+}^{n}\right)$ si ha

$$
\begin{aligned}
& <a D^{\alpha} f, \overline{D_{t}^{m_{n}} \varphi}>=\sum_{j=1}^{m_{n}}\left(\begin{array}{c}
m_{n} \\
j
\end{array}\right)<D_{a}^{i} a \cdot D_{t}^{m_{n}-j} D^{\alpha} f, \bar{\varphi}> \\
& \left.+\left\langle D^{\alpha} A f, \bar{\varphi}\right\rangle-\underset{\substack { \Sigma \\
\begin{subarray}{c}{\alpha, q>\leq m \\
\mu_{n}<m_{n}{ \Sigma \\
\begin{subarray} { c } { \alpha , q > \leq m \\
\mu _ { n } < m _ { n } } }\end{subarray}}{\Sigma} \Sigma_{\beta \leq \alpha}\left(\begin{array}{l}
\alpha \\
\beta
\end{array}\right)<D^{\alpha-\beta} a_{\mu \alpha} \cdot D^{\mu} D^{\beta} f, \bar{\varphi}\right\rangle \\
& -\sum_{\beta<x}\left(\begin{array}{l}
\alpha \\
\beta
\end{array}\right)<D^{\alpha-\beta} a \cdot D_{t}^{m_{n}} D^{\beta} f, \bar{\varphi}>.
\end{aligned}
$$

Dalla (5.7) scritta per $\alpha=(0, \ldots, 0, r)$ si deduce evidentemente che esiste una costante $M$ tale che si ha

$$
\left|\left(a D_{t}^{r} f, D_{t}^{m_{n}} \varphi\right)\right| \leq M \underset{|\mu| \leq m_{n}-1}{\Sigma}\left\|D^{\mu} \varphi\right\|_{L^{2}(\Sigma(\rho))} \quad \forall \varphi \in C_{0}^{\infty}(\Sigma(\rho)-\partial \Sigma(\rho)),
$$

dove il simbolo (, ) denota il prodotto scalare in $L^{2}(\Sigma(\rho))$.

La (5.8) implica, per noti risultati (cfr., ad es., il lemma 9.3 di \$. AGMoN [2]), che $D_{t}\left(a D_{t}^{r} f\right) \in L^{2}\left(\Sigma\left(\rho^{\prime}\right)\right)$ per ogni $\rho^{\prime}<\rho$.

Resta così dimostrato che sussiste la (5.4).

Per stabilire poi la (5.6), consideriamo la (5.7) scritta con $\left|\alpha^{\prime}\right|=h$ e $\alpha_{n}^{4}=r$. Da tale relazione, usufruendo delle ipotesi, si deduce facilmente che esiste una costante $M_{1}$ tale che si ha

$$
\left|\left(a D_{y}^{\alpha^{\prime}} D_{t}^{r} f, D_{t}^{m}{ }^{n} \varphi\right)\right| \leq M_{1} \underset{|\mu| \leq m_{m^{\prime}-1}}{\Sigma}\left\|D^{\mu} \varphi\right\|_{L^{2}(\Sigma(\rho))} \quad \forall \varphi \in C_{0}^{\infty}(\Sigma(\rho)-\partial \Sigma(\rho))
$$

Dalla (5.9) si deduce, come nel caso precedente, che $D_{t}\left(a D_{y}^{\alpha^{\prime}} D_{t}^{r} f\right) \in L^{2}\left(\Sigma\left(\rho^{\prime}\right)\right)$ per ogni $\rho^{\prime}<\rho$, e quindi, come volevasi, che sussiste la (5.6). Si ha cosi la tesi. 
Consideriamo ancora gli operatori $A(x, D)$ e $B_{j}(y, D)$ definiti al n. 3 .

Dai teoremi 4.1 e 5.1 consegue orviamente il seguente

Corolianio 5.1. - Siano verificate le ipotesi $\left.\left.\beta_{1}\right), \beta_{2}\right)$ e sia $F=\left(f, g_{1}, \ldots, g_{v}\right)$ un vettore di classe $L^{2}\left(R_{+}^{n}\right) \times \prod_{j=1}^{y} H^{-m+p_{j}+q_{n}{ }^{2}}\left(R^{n-1}\right)$ e tale che

$$
P^{*} F \in C^{\infty}\left(\bar{\Gamma}\left(\rho_{0}\right)\right) \text { per un } \rho_{0}>0 \text {. }
$$

Esiste, per ogni intero $k \geq 0$, un numero positivo $\rho<p o$ tale che si $h \alpha$

$$
\begin{gathered}
D^{\alpha} f \in L^{2}(\Sigma(\rho)), \quad|\alpha| \leq k, \\
D_{y}^{\tau} g_{j} \in L^{2}(\sigma(\rho)), \quad|\tau| \leq k, \quad j=1, \ldots, \text { v. }
\end{gathered}
$$

Richiamiamo ora le seguenti definizioni (cfr. T. Matsuzawa [21], [22] e C. Parenti [29] (6)):

Defintzrone 5.1. - Si dice che l'operatore $A(x, D)$ è propriamente quasiellittico di tipo $\vee$ su un sottinsieme $\mathscr{D}$ di $\left|x \in R^{n}\right| x_{n}=0 \mid$, se per ogni $x_{0} \in \mathscr{D}$ l'operatore a coefficienti costanti $A_{0}\left(x_{0}, D\right)$ verifica l'ipotesi $\left.\alpha_{1}\right)$ con $\vee$ indipendente anche da $x_{0}$.

Definizione 5.2. - Se $A(x, D)$ è propriamente quasi-ellittico di tipo $v$ su $\mathscr{D}$, si dice che gli operatori $B_{1}(y, D), \ldots, B_{v}(y, D)$ verificano la condizione complementare rispetto ad $A(x, D)$ su $\mathscr{D}$, se per ogni $x_{0}=\left(y_{0}, 0\right) \in \mathscr{D}$ gli operatori a coefficienti costanti $B_{01}\left(y_{0}, D\right), \ldots, B_{0_{\nu}}\left(y_{0}, D\right)$ verificano la condizione complementare rispetto ad $A_{0}\left(x_{0}, D\right)$.

Osservazione 5.1. - Se $\mathscr{D}$ è connesso e $A(x, D)$ è quasi-ellittico in $\mathscr{D}$, dalla continnità dei coefficienti segue evidentemente che $A(x, D)$ risulta propriamente quasi-ellittico su $\mathfrak{D}$ se, per un particolare punto $x_{0} \in \mathfrak{D}$, l'operatore $A_{0}\left(x_{0}, D\right)$ soddisfa l'ipotesi $\left.\alpha_{1}\right)$. E inoltre noto (efr., ad es., A. Caval. LUCOI a pag. 146 di [8] ed il lemma 1 di [9]) che, nel caso $n>2$ e se $\mathscr{D}$ ò

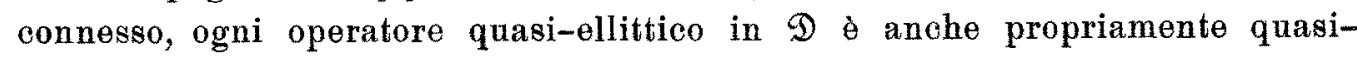
ellittico su $\mathfrak{D}$.

(6) Avvertiamo che è stato possibile prendere visione del lavoro [29] di C. Parentr solo a stesura ultimata del presente lavoro. 
Dalla ipoellitticità degli operatori quasi-ellittici e dal corollario 5.1 si deduce con note considerazioni il seguente

CoRollario 5.2. - Assegnato un numero reale $\rho_{0}>0$, supponiamo che $A(x, D)$ sia quasi-ellittico in $\Sigma\left(\rho_{0}\right)$, propriamente quasi-ellittico di tipo v su $\sigma\left(\rho_{0}\right)$ e che gli operatori $B_{1}(y, D), \ldots, B_{y}(y, D)$ verifichino la condizione complementare rispetto ad $A(x, D)$ su $\sigma\left(\rho_{0}\right)$.

Allora per ogni $F=\left(f, g_{1}, \ldots, g_{v}\right) \in L^{2}\left(R_{+}^{n}\right) \times \prod_{j=1}^{\vee} H^{-m+p_{j}+q_{n} l^{2}}\left(R^{n-1}\right)$ e tale che

$$
P^{*} F \in O^{\infty}\left(\overline{\Gamma\left(\rho_{0}\right)}\right)
$$

si ha per $p<\rho_{0}$

$$
f \in C^{\infty}(\bar{\Sigma}(\rho)), \quad g_{j} \in C^{\infty}(\overline{\sigma(\rho))} \quad(j=1, \ldots, \nu) .
$$

\section{6. - La formula di Green.}

In questo numero per ogni $x \in R^{n}$ porremo ancora $x=(y, t)$, dove $y \in R^{n-1}$ e $t \in R^{1}$.

Indicheremo con $\sigma$ un insieme aperto e connesso di $R^{n-1}$, contenente l'origine, $\theta$ con $\Sigma$ l'insieme $\{(y, t) \mid y \in \sigma$ e $0 \leq t<1\}$.

Inoltre indicheremo con $\tau=\left(\tau_{1}, \ldots, \tau_{n}\right)$ un'assegnata $n$-pla di numeri reali positivi, con $\delta(y, t)$ un'assegnata funzione di classe $C^{0}(\bar{\Sigma})$ e positiva in $\Sigma$; porremo $\tau^{\prime}=\left(\tau_{1}, \ldots, \tau_{n-1}\right)$ e $\delta_{0}(y)=\delta(y, 0)$.

Assegnamo in $\Sigma$ un operatore differenziale lineare della forma

$$
L=L(x, D)=\sum_{<\mu, q>\leq m} l_{\mu}(x) D^{\mu} .
$$

Definizione 6.1. - Diremo che $L$ è un operatore quasi-ellittico di classe $(r, s)$, dove $r \in J(\mathscr{N})$ e $s \in R^{1}$, se:

1) le derivate $D^{x} l_{\mu} \in C^{0}(\Sigma)$ per $\langle\alpha, q\rangle \leq<\mu, q>+r$, ed esiste una costante $M$ tale che si abbia per $\langle\alpha, q>\leq<\mu, q>+r$ e per $x \in \Sigma$

$$
\mid \delta^{s+<\alpha, \tau>-<\mu, \tau>D^{\alpha} l_{\mu} \mid \leq M}
$$

2) $L$ ə̀ quasi-ellittico in $\Sigma$;

3) la funzione $\delta^{s-m_{n} \tau_{n}} l_{\left(0, \ldots, 0, m_{n}\right)}$ əे continua e $\neq 0$ su $\bar{\Sigma} \cap\{t=0\}$. 
Assegnamo inoltre un sistema di operatori differenziali tangenziali definiti su $\Sigma \cap\{t=0\}$ e della forma

$$
\Theta_{j k}=\sum_{\left\langle\eta, q^{\prime}\right\rangle \leq q_{n}(j-k)} \Theta_{j \eta^{k}}(y) D_{y}^{n}, \quad k=0, \ldots, j \text { e } j=0, \ldots, m_{n}-1
$$

avendo posto $q^{\prime}=\left(q_{1}, \ldots, q_{n-1}\right)$ e, per ogni $(n-1)$-pla di interi non negativi $\eta=\left(\eta_{1}, \ldots, \eta_{n-1}\right),<\eta, q^{\prime}>=\eta_{1} q_{1}+\ldots+\eta_{n-1} q_{n-1}$.

Defintzione 6.2. - Diremo che gli operatori $\theta_{j k}$ costituiscono un sistema di classe $(r, s)$, dove $r \in J(\mathscr{T K})$ e $s \in R^{1}$, se:

a) le derivate $D_{y}^{\beta} \Theta_{j n k}(y) \in C^{0}(\sigma)$ per $<\beta, q^{\prime}>\leq q_{n}\left(m_{n}-j\right)+r$, ed esiste una costante $M_{0}$ tale che si abbia per $<\beta, q^{\prime}>\leq q_{n}\left(m_{n}-j\right)+r$ e per $y \in \sigma$

$$
\left|\delta_{0}^{s+<\beta, \tau^{\prime}>-<\eta_{1} \tau^{\prime}>+\tau_{n}(j-k)} D_{y}^{\beta} \Theta_{j \eta^{k}}\right| \leq M_{0}
$$

b) le funzioni $\delta_{\theta}^{s} \Theta_{j j}$ sono continue e $\neq 0$ su $\bar{\sigma}$.

Sia poi $\left\{\boldsymbol{F}_{j}\right\}_{j=0}^{m_{n}-1}$ un sistema di $\boldsymbol{m}_{n}$ operatori differenziali lineari di frontiera definiti su $\Sigma \cap\{t=0\}$ e della forma

$$
F_{j}=\sum_{\left\langle n, q^{\prime}>+k q_{n} \leq j q_{n}\right.} F_{j \eta k}(y) D_{y}^{n} D_{t}^{k}=\sum_{k=0}^{j} F_{j k} D_{t}^{k}, \quad j=0, \ldots, m_{n}-1
$$

Definizione 6.3. - Diremo che $\left\{F_{j}\right\}_{j=0}^{m_{n}-1}$ è un sistema di DrRichlet di classe $(r, s)$, se gli operatori tangenziali $F_{j k}$ costituiscono un sistema di classe $(r, s)$.

LemMa 6.1. - Se $\left\{F_{j}\right\}_{j=0}^{m_{n}-1}$ è un sistema di Dirichlet di classe $(r, s)$, esiste un sistema $\Phi_{j k}, 0 \leq k \leq j \leq m_{n}-1$, di operatori tangenziali di classe $(r,-s)$ tale che si ha

$$
D_{t}^{j}=\sum_{k=0}^{j} \Phi_{j k} F_{k}, \quad j=0, \ldots, m_{n}-1
$$

La dimostrazione di questo lemma è analoga alla dimostrazione del lemma 4.1 di [34], a cui rinviamo. 
Lвмма 6.2. - Supponiano che $\left\{F_{j}\right\}_{j=0}^{m_{n} n^{-1}}$ sia un sistema di Dirichlet di classe $(r, s)$, per un $r \in J(\mathscr{N})$ e per un $s \in R^{1}$, e che $i$ coefficienti $d i F_{j}$ siano $d i$ classe $C^{\infty}(\sigma)$.

Allora comunque si assegnano le funzioni $\varphi_{j} \in C_{0}^{\infty}(\sigma), j=0, \ldots, m_{n}-1$, esiste $v \in C_{0}^{\infty}(\Sigma)$ tale che si ha

$$
F_{j} v=\varphi_{j} \text { per } t=0, \quad j=0, \ldots, m_{n}-1
$$

Applicando un noto procedimento (cfr., ad es., la dimostrazione del lemma 2.2 del cap. 2 di J.L. LTons-E. Magenes [20]), poniamo

$$
\psi_{k}=\sum_{h=0}^{k} \Phi_{k h} \varphi_{h}, \quad k=0, \ldots, m_{n}-1
$$

dove i $\Phi_{k k}$ sono definiti dal lemma 6.1.

Siccome, nelle ipotesi poste, i coefficienti degli operatori $\Phi_{k h}$ sono di classe $C^{\infty}(\sigma)$ (ofr. la (4.7) di [34] che fornisce le espressioni dei $\Phi_{k h}$ ), si ha che, se le $\varphi_{j} \in C_{0}^{\infty}(\sigma)$, le $\psi_{k} \in C_{0}^{\infty}(\sigma)$.

$\mathrm{Ne}$ segue, per noti risultati, che esiste $v \in C_{0}^{\infty}(\Sigma)$ tale che

$$
D_{t}^{k} v=\psi_{k} \text { per } t=0, \quad k=0, \ldots, m_{n}-1
$$

Si ha allora

$$
F_{j} v=\sum_{k=0}^{j} F_{j k} D_{t}^{k} v=\sum_{h=0}^{j}\left(\sum_{k=h}^{j} F_{j k} \Phi_{k h}\right) \varphi_{h}
$$

D'altra parte, in conseguenza delle (6.3) e (6.4), si ha

$$
\sum_{k=h}^{j} F_{j h} \Phi_{k h}=\delta_{j h}, \quad 0 \leq h \leq j \leq m_{n}-1
$$

Dalle (6.6) e (6.7) segue la (6.5), e quindi si ha la tesi.

Sussiste inoltre il seguente lemma che costituisce nna generalizzazione del lemma 4.2 di [34]:

Lemma 6.3. - Supponiamo che L sia un operatore quasi-ellittico di classe $\left(r, s^{\prime}\right)$ e che $\left\{F_{j}\right\}_{j=0}^{m_{n}-1}$ sia un sistema di Dirichlet di classe $\left(r+q_{n}\left(m_{n}-1\right), s^{\prime \prime}\right)$. 
Esiste un sistema di Dirichlet $\left\{G_{j}\right\}_{j=0}^{m_{n}-1}$ di classe $\left(r, s^{\prime}-s^{\prime \prime}-m_{n} \tau_{n}\right)$ tale che per ogni coppia di funzioni u e v, una di classe $C^{\infty}(\Sigma)$ e l'altra di classe $C_{0}^{\infty}(\Sigma)$, sussiste la formula di Green

$$
\int_{\Sigma} L u \cdot \bar{v} d y d t-\int_{\Sigma} u \cdot \overline{L^{*} v} d y d t=\sum_{j=0}^{m_{n}-1} \int_{\sigma}\left[F_{j} u \cdot \overline{G_{m_{n}-j-1} v}\right]_{t=0} d y
$$

dove $L^{*}$ denota l'operatore aggiunto formale di $L$.

Infatti, assegnate le funzioni $u$ e $v$, una di classe $C^{\infty}(\Sigma)$ e l'altra di classe $C_{0}^{\infty}(\Sigma)$, con delle integrazioni per parti si prova che si ha

$$
\int_{\Sigma} L u \cdot \vec{v} d y d t=\int_{\Sigma} u \cdot \overline{L^{*} v} d y d t+\sum_{j=0}^{m_{n}-1} \int_{\sigma}\left[D_{t}^{j} u \cdot \overline{N_{j} v}\right]_{t=0} d y
$$

dove

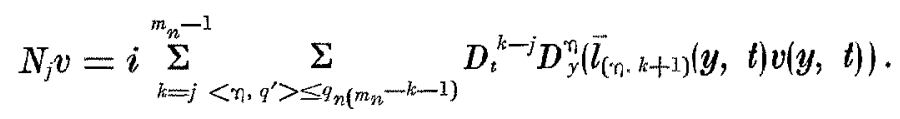

Consideriamo gli operatori tangenziali $\Phi_{j k}$ definiti dal lemma 6.1 . Indichiamo con $\Phi_{j k}^{*}$ l'operatore aggiunto formale di $\Phi_{j k}$ e poniamo

$$
G_{j}=\sum_{h=m_{n}-j-1}^{m_{n}-1} \Phi_{h, m_{n}-j-1}^{*} N_{h}, \quad j=0, \ldots, m_{n}-1
$$

Si verifica facilmente che gli operatori $G_{j}$ definiti dalla (6.11) soddisfano le condizioni richieste dal lemma.

Assegnamo ora gli interi $p_{1}, \ldots, p_{\nu}, r_{1}, \ldots, r_{m_{n}-v}$ tali che $\left\{p_{1}, \ldots, p_{y}, r_{1}\right.$, $\left.\ldots, r_{m_{n}-y}\right\}=\left\{0,1, \ldots, m_{n}-1\right\}, \theta$, supponendo verificate le condizioni del lem. ma 6.3 , poniamo

$$
\begin{gathered}
B_{j}(y, D)=F_{p_{j}}, \quad C_{j}(y, D)=F_{r_{i}}, \\
B_{j}^{\prime}(y, D)=G_{m_{n}-r_{j}-1}, \quad C_{j}^{\prime}(y, D)=-G_{m_{n}-p_{j}-1} .
\end{gathered}
$$


Con tali posizioni, dalla (6.8) si ha per ogni coppia di funzioni $u$ e $v$ di classe $C_{0}^{\infty}\left(\overline{R_{+}^{n}}\right)$ ed a supporto compatto in $\Sigma$

$$
\begin{aligned}
\int_{R_{+}^{n}} L u \cdot \bar{v} d y d t- & \int_{R_{+}^{n}} u \cdot \overline{L^{*} v} d y d t=\sum_{j=1}^{m_{n}-v} \int_{R^{n-1}}\left[C_{j} u \cdot \overline{\left.B_{j}^{\prime} v\right]_{t=0}} d y\right. \\
& -\sum_{j=1}^{\nu} \int_{R^{n-1}}\left[B_{j} u \cdot C_{j}^{\prime} v\right]_{t=0} d y .
\end{aligned}
$$

Osserviamo che se l'operatore $L(x, D)$ e propriamente quasi-ellittico di tipo y su $\sigma$, allora l'operatore $L^{*}(x, D)$ è propriamente quasi-ellittico di tipo $m_{n}-v$ su $\sigma$.

Sussiste il seguente

Lemma 6.4. - Siano verificate le ipotesi del lemma 6.3 ed inoltre $L(x, D)$ sia propriamente quasi-ellittico di tipo \& su $\sigma$.

Allora gli operatori $B_{1}(y, D), \ldots, B_{v}(y, D)$ verificano la condizione complementare rispetto a $L(x, D)$ su a se e solo se gli operatori $B_{1}^{\prime}(y, D), \ldots, B_{m_{n}-\nu}^{\prime}(y, D)$ verificano la condizione complementare rispetto a $L^{*}(x, D) s u \sigma$.

La dimostrazione di questo lemma si ottiene adattando al nostro caso un noto procedimento utilizzato per dimostrare un risultato analogo nel caso ellitico, ed esposto, ad es., nel n. 4.3 del cap. 2 di J. L. Lions-E. Magenes [20].

Ne diamo un accenno di dimostrazione, allo scopo di indicare alcuni accorgimenti di carattere formale che rendono possibile l'applicazione al nostro caso di detto procedimento relativo al caso ellittico.

Siano $L_{0}, L_{0}^{*}, B_{0 j}, \ldots$ le parti principali rispettivamente di $L, L^{*}, B_{j}, \ldots$

Possiamo evidentemente limitarei a dimostrare che gli operatori $B_{01}(0, D)$, $\ldots, B_{0}(0, D)$ verificano la condizione complementare rispetto a $L_{0}(0, D)$ se $\theta$ solo se gli operatori $B_{01}^{\prime}(0, D), \ldots, B_{0, m_{n}-,}^{\prime}(0, D)$ verificano la condizione complementare rispetto a $L_{0}^{*}(0, D)$.

Poniamo, per $\lambda$ reale $>1$,

$$
w_{\lambda}(y, t)=w\left(\lambda^{q_{1}} y_{1} \ldots, \lambda^{q_{n-1}} y_{n-1}, \lambda q_{n} l\right)
$$

Osserviamo che se le funzioni $u$ e $v$ sono di classe $C_{0}^{\infty}\left(\overline{R_{+}^{n}}\right)$ ed a supporto compatto in $\Sigma, u_{\lambda}$ e $v_{\lambda}$ sono ancora funzioni di classe $C_{0}^{\infty}\left(R_{+}^{n}\right)$ ed a supporto compatto in $\Sigma$. 
Applicando allora la (6.12) a $\boldsymbol{u}_{\lambda}$ e $\boldsymbol{v}_{\lambda}$, operando nella formula cosi ottenuta il cambiamento di variabili $y_{i}^{\prime}=\lambda^{q_{i}} y_{i}, t^{\prime}=\lambda q_{n} t$ e passando al limite per $\lambda \rightarrow \infty$ si ottiene la formula

$$
\begin{gathered}
\int_{R_{+}^{n}} L_{0}(0, D) u(y, t) \cdot \overline{v(y, t)} d y d t-\int_{R_{+}^{n}} u(y, t) \cdot \overline{L_{0}^{*}(0, D) v(y, t)} d y d t \\
\quad=\left.\left.\sum_{j=1}^{m} \int_{R^{n-1}} C_{0 j}(0, D) u(y, t)\right|_{t=0} \cdot \overline{B_{0 j}^{\prime}(0, D) v(y, t)}\right|_{t=0} d y \\
-\left.\quad \sum_{j=1}^{y} \int_{R^{n-1}} B_{0 j}(0, D) u(y, t)_{t=0} \cdot \overline{C_{0 j}^{\prime}(0, D) v(y, t)}\right|_{t=0} d y,
\end{gathered}
$$

nell'ipotesi che $u$ e $v$ siano di classe $C_{0}^{\infty}\left(\overline{R_{+}^{n}}\right)$ ed a supporto compatto in $\Sigma$.

Se poi $u$ e $v$ sono delle funzioni qualunque di classe $C_{0}^{\infty}\left(\overline{R_{+}^{n}}\right)$, per $\lambda$ sufficientemente grande $u_{\lambda}$ e $v_{\lambda}$ hanno supporto compatto in $\Sigma$. Applicando allora la (6.13) a tali funzioni $u_{\lambda}$, $v_{\lambda}$ ed operando il cambiamento di variabili $y_{i}^{\prime}=\lambda_{i} y_{i}, t^{\prime}=\lambda^{q} u t$, si deduce che la (6.13) sussiste per ogni $u, v \in C_{0}^{\infty} \overline{\left(R_{+}^{n}\right.}$.

Tenendo allora presenti i lemmi 2.1 e 2.2, si ottiene la tesi ripetendo i ragionamenti tenuti nel eap. 2 di [20] per dedurre dalla (4.20) la tesi del teorema 2.2 .

\section{Capitolo II}

\section{Problemi quasi-ellittici in un dominio limitato.}

\section{7. - Preliminari; spazi di Sobolev con peso.}

Nel seguito indicheremo con $n_{1}, \ldots, n_{t}$ dei numeri interi positivi tali che

$$
n_{1}+\ldots+n_{t}=n
$$

Supporremo che sia 


$$
m_{n_{0}+\ldots+n_{i-1}+1}=\ldots=m_{n_{0}+\ldots+n_{i}}=m^{(i)}, \quad i=1, \ldots, t,
$$

dove $n_{0}=0$, e porremo

$$
q^{(i)}=\frac{m}{m^{(i)}}, \quad i=1, \ldots, t
$$

Inoltre indicheremo con $R^{(i)}, i=1, \ldots, t$, lo spazio reale euclideo a $n_{i}$ dimensioni di punto $x^{(i)}=\left(x_{1}^{(i)}, \ldots, x_{n_{i}}^{(i)}\right)$, e per ogni $x \in R^{n}$ porremo $x=\left(x^{(1)}\right.$, $\left.\ldots, x^{(t)}\right)$, dove $x^{(i)} \in R^{(i)}$.

Se $\mathfrak{D}$ è un insieme misurabile di $R^{n}$, per ogni $u \in L^{p}(\mathfrak{D})$ porremo

$$
|\boldsymbol{u}|_{p, \mathfrak{D}}=\|\boldsymbol{u}\|_{L^{p}}(\mathfrak{D})
$$

Sussiste il seguente lemma contenuto nel corollario I di [35]:

Lemma 7.1. - Sia $\mathscr{D}=\mathscr{D}^{(1)} \times \ldots \times \mathfrak{D}^{(t)}$, dove $\mathfrak{D}^{(i)}, i=1, \ldots, t$, è un insieme aperto e limitato di $R^{(i)}$, dotato della proprietì di cono, e sia $p$ un numero reale $>1$.

Per ogni distribuzione $u$ su $\mathfrak{D}$ tale che $u \in L^{P}(\mathfrak{D}), D_{x_{k}}^{m_{k}} u \in L^{p}(\mathfrak{D}), k=1, \ldots, n$, per ogni n-pla di interi non negativi a tale che $<x, q><m$ e per ogni $a \in[<\alpha, q>/ m, 1]$ sussiste la limitazione

$$
\left.i D^{x} \boldsymbol{u}\right|_{p^{\prime}, \mathfrak{D}} \leq c\left[\left(\sum_{k=1}^{n}\left|D_{x_{k}}^{m_{k}} \boldsymbol{u}\right|_{p, \mathfrak{D}}\right)^{a} \cdot|\boldsymbol{u}|_{p, \mathfrak{D}}^{1-a}+|\boldsymbol{u}|_{p, \mathfrak{D}}\right]
$$

essendo: c una costante indipendente $d a u, p^{\prime}$ un numero $\geq p$ e tale che

$$
\frac{1}{p^{\prime}} \geq \frac{1}{p}-\frac{a m-<\alpha, q>}{|q|}
$$

dove va escluso il segno di uguaglianza nei seguenti due casi: 1$)<\alpha, q\rangle \leq$ $\leq m-|q| / p$ e $a=1$; 2) $m=|q| / p$ e $a \neq<\alpha, q\rangle / m$.

Assegnamo, per ogni $i \in\{1, \ldots, t\}$, un insieme aperto e limitato $\Omega^{(i)}$ di $R^{(i)}$ e un insieme chiuso $S^{(i)}$, eventualmente vuoto, di punti di $\partial \Omega^{(i)}$, supponendo che sia verificata la seguente condizione: 
$\left.a_{0}\right)$ Se $S^{(i)} \neq 2 \Omega^{(i)}$, esiste un ricoprimento di $\partial \Omega^{(i)}-S^{(i)}$ costituito da un numero finito $I_{1}^{(i)}, \ldots, I_{r_{i}}^{(i)}$ di aperti di $R^{(i)}$, disgiunti da $S^{(i)}$ e tali che ad ogni $I_{k}^{(i)}$ sia possibile associare un omeomorfismo $x \rightarrow \alpha_{k}^{(i)}(x)$ di classe $C^{\infty}$ di $\overline{I_{k}^{(i)}}$ sul eilindro $\left\{y \in R^{(i)} \mid y_{1}^{2}+\ldots+y_{n_{i}-1}^{2} \leq 1,-1 \leq y_{n_{i}} \leq 1\right\}$, il quale trasformi $I_{k}^{(i)} \cap \Omega^{(i)}$ nell'insieme $\left.y \in R^{(i)} \mid y_{1}^{2}+\ldots+y_{n_{i}-1}^{2}<1,0<y_{n_{i}}<1\right\}$ e $I_{k}^{(i)} \cap\left(\partial \Omega^{(i)}-S^{(i)}\right)$ nell'insieme $\left\{y \in R^{(i)} \mid y_{1}^{2}+\ldots+y_{n_{i}-1}^{2}<1, y_{n_{i}}=0\right\}$; inoltre sia soddisfatta la seguente condizione di compatibilità: se $I_{h}^{(i)} \cap I_{k}^{(i)} \neq \varnothing$, esiste un omeomorfismo $\beta_{h k}^{(i)}$ di classe $C^{\infty}$ ed a jacobiano positivo di $\alpha_{h}^{(i)}\left(I_{h}^{(i)} \cap I_{k}^{(i)}\right)$ su $\alpha_{k}^{(i)}\left(I_{h}^{(i)} \cap I_{k}^{(i)}\right)$ tale $\operatorname{ch} \theta$

$$
\alpha_{k}^{(i)}(x)=\beta_{h(i)}^{(i)}\left(\alpha_{h}^{(i)}(x)\right) \quad \forall x \in I_{h}^{(i)} \cap I_{k}^{(i)}
$$

Riprendendo alcune notazioni definite nell'introdazione, poniamo

$$
\begin{gathered}
\Omega=\Omega^{(1)} \times \ldots \times \Omega^{(i)}, \\
\partial_{i} \Omega=\Omega^{(1)} \times \ldots \times \Omega^{(i-1)} \times\left(\partial \Omega^{(i)}-S^{(i)}\right) \times \Omega^{(i+1)} \times \ldots \times \Omega^{(t)}, \quad i=1, \ldots, t, \\
S=\partial \Omega-\bigcup_{i=1}^{i} \partial_{i} \Omega,
\end{gathered}
$$

e indichiamo con $\mathfrak{d}$ l'insieme dei numeri $i \in\left\{1, \ldots, t \mid\right.$ tali che $\partial_{i} \Omega \neq \varnothing$.

Osservazione 7.1. - La condizione sopra imposta agli insiemi $\Omega^{(i)}$ implica, evidentemente, l'esistenza di un ricoprimento di $\partial_{i} \Omega$ costituito da un nu. mero finito $\mathscr{Q}_{i 1}, \ldots, \mathscr{Q}_{i h_{i}}$ di aperti di $R^{n}$, disgiunti da $\partial \Omega-\partial_{i} \Omega$ e tali che ad ogni $\mathscr{W}_{i k}$ si possa associare un omeomorfismo $x \rightarrow \theta_{i k}(x)$ di classe $C^{\infty}$ di $\mathscr{Q}_{i k}$ su un insieme aperto e connesso $\Gamma$ di $R^{n}$, contenuto nell'insieme $\left\{y=\left(y^{(1)}, \ldots\right.\right.$, $\left.\left.y^{(t)}\right) \in R^{n}|| y^{(j)} \mid<1, j=1, \ldots, t\right\}$ e contenente l'origine, in modo che siano soddisfatte le seguenti condizioni:

$\left.r_{1}\right) \theta_{i k}$ trasforma $\mathscr{Q}_{i k} \cap \Omega$ nell'insieme $\Sigma^{(i)}=\left\{y=\left(y^{(1)}, \ldots, y^{(i)}\right) \in R^{n} \mid\right.$ $y_{n_{i}}^{(i)}>0 i \cap \Gamma$ e $\mathcal{Q H}_{i k} \cap \partial_{i} \Omega$ nell'insieme $\sigma^{(i)}=\left\{y=\left(y^{(1)}, \ldots, y^{(i)}\right) \in R^{n} \mid y_{n_{i}}^{(i)}=0\right\} \cap \Gamma$;

$\left.r_{2}\right)$ posto $y=\theta_{i k}(x)$, riesce $\partial y_{h}^{(r)} / \partial x_{j}^{(s)}=0$ per $r \neq s$ e $\partial y_{h} / \partial x_{h}>0$ (7);

$r_{3}$ ) è soddisfatta una condizione di compatibilitá del tipo di quella descritta nell'ipotesi $a_{0}$ ).

(7) Osserviamo che tale condizione implica che la trasformazione $y=\theta_{i n}(x)$ risulta, nel senso di A. Cavallucc [9], stabile per ogni polinomio quasi-ellitico della forma $P(\xi)=$ $=\underset{<\alpha, Q>\leq m}{\Sigma} \alpha_{\alpha} \xi^{\alpha}$ o quindi che $\partial_{i} \Omega$ risulta, ancora nel senso di [9], una superficie normale per $P(\xi)$. 
Indicheremo:

con $\stackrel{\circ}{W}^{k, p}(\Omega), k \in J(\mathscr{R})$ e $p$ reale $\geq 1$, la classe delle funzioni $u \in W^{k, p}(\Omega)\left(^{8}\right)$ ed a supporto compatto in $\bar{\Omega}-S$;

con $C^{\infty}(\bar{\Omega})$ la classe delle funzioni $u \in C^{\infty}(\bar{\Omega})$ ed a supporto compatto in $\bar{\Omega}-S ;$ ciò porremo $\stackrel{\circ}{C^{\infty}}(\bar{\Omega})=C_{0}^{\infty}(\bar{\Omega}-S)$.

LEMma 7.2. - Qualunque siano $k \in J(\mathfrak{O K})$ e $p$ reale $\geq 1, \check{C}^{\infty}(\overline{\mathbf{\Omega}})$ è denso in $\stackrel{\circ}{W}^{k, p}(\Omega)$.

Infatti, sia $u$ una funzione di classe $\dot{W}^{k, p}(\Omega)$ e sia $T$ il suo supporto.

Consideriamo gli aperti $\mathscr{L}_{i h}$ definiti nell'osservazione 7.1 e due aperti $\mathcal{Q}_{0}, \mathscr{Q}_{\mathrm{I}}$ di $R^{n}$ tali che

$$
\overline{\mathcal{Q}}_{0} \subset \Omega, S \subset \mathcal{Q R}_{1}, \mathscr{Q}_{1} \cap \bar{\Omega} \subset \bar{\Omega}-T
$$

e tali inoltre che gli insiemi $\mathscr{W}_{i h}\left(h=1, \ldots, h_{i}, i \in \mathfrak{J}\right), \mathscr{Q}_{0}$ e $\mathscr{Q}_{1}$ costituiscano un ricoprimento di $\bar{\Omega}$.

Assegnamo quindi una partizione dell'unità su $\bar{\Omega}$ relativa al suddetto ricoprimento e costituita di funzioni $\alpha_{i h}, \alpha_{0}, \alpha_{1}$ di classe $C_{0}^{\infty}\left(R^{n}\right)$ e tali che

$$
\operatorname{supp} \alpha_{i h} \subset \mathcal{Q}_{i h}, \quad \operatorname{supp} \alpha_{0} \subset \mathcal{Q R}_{0}, \quad \operatorname{supp} \alpha_{1} \subset \mathcal{Q H}_{1}
$$

Facendo nso dell'operatore regolarizzante definito al n. 1.3 del cap. 2 di J. Nwöas [26] (cfr. anche il n. 1.2 di L. Hörmander [18] e il n. 1 di S. AgMoN [2]) e tenendo conto dell'osservazione 7.1, con note considerazioni (ofr., ad es., la dimostrazione del teorema 3.1 del cap. 2 di [26]) si dimostra ehe, per ogni $i$ ed $h$, esiste una successione $\left.; u_{i h n}\right)$ di funzioni di classe $C^{\infty}(\bar{\Omega})$ ed aventi supporto contenuto in $\mathcal{Q}_{i h} \cap \bar{\Omega}$ convergente ad $\alpha_{i h} u$ in $W^{k, p}(\Omega)$, ed esiste una sucessione $\left\{u_{0 n} \mid\right.$ di funzioni di classe $C_{0}^{\infty}(\Omega)$ convergente ad $\alpha_{0} u$ in $W^{k_{p} p}(\Omega)$.

Posto allora

$$
\boldsymbol{u}_{n}=\sum_{i \in \mathfrak{J}} \sum_{k=1}^{h_{i}} \boldsymbol{u}_{i h n}+\boldsymbol{u}_{0 n}
$$

si ha evidentemente che $u_{n} \in \check{C}^{\infty}(\bar{\Omega})$ e che la successione $\left\{u_{n}\right\}$ converge ad $u$ in $W^{k, p}(\Omega)$. Ne segue la tesi.

(8) Per la definizione di $W^{k, p}(\Omega)$ ofr. il n. 1 (def. 1.2). 
Indicheremo: con $\tau=\left(\tau_{1}, \ldots, \tau_{n}\right)$ un'assegnata $n$-pla di numeri reali $>1$ e tali che

$$
\tau_{n_{0}+\ldots+n_{i-1}+1}=\ldots=\tau_{n_{0}+\ldots+n_{i}}=\tau^{(i)}, \quad i=1, \ldots, t,
$$

con $d$ un assegnato numero reale $>1$ e $\geq \frac{1}{\sqrt{t}} \sup _{x \in \bar{\Omega}}$ dist $(x, S)$, e porremo per ogni $x_{0} \in \bar{\Omega}$

$$
\begin{gathered}
\rho\left(x_{0}\right)=\frac{1}{d \sqrt{t}} \text { dist }\left(x_{0}, S\right), \\
Q\left(x_{0}\right)=\left\{x \in R^{n}|| x^{(i)}-x_{0}^{(i)} \mid<\rho^{\tau^{(i)}}\left(x_{0}\right), i=1, \ldots, t\right\}, \\
I\left(x_{0}\right)=\Omega \cap Q\left(x_{0}\right), \quad \Gamma\left(x_{0}\right)=\partial \Omega \cap Q\left(x_{0}\right) .
\end{gathered}
$$

Si verifica facilmente che riesce

$$
\begin{gathered}
\left|\rho\left(x^{\prime}\right)-p\left(x^{\prime \prime}\right)\right| \leq \frac{1}{d \sqrt{t}}\left|x^{\prime}-x^{\prime \prime}\right| \quad \forall x^{\prime}, x^{\prime \prime} \in \Omega, \\
\frac{d-1}{d} p\left(x_{0}\right)<\rho(x)<\frac{d+1}{d} \rho\left(x_{0}\right) \quad \forall x \in I\left(x_{0}\right), \\
\overline{\Gamma\left(x_{0}\right)} \cap S=\emptyset .
\end{gathered}
$$

Dalla (7.4) si deduce che, posto

$$
\Gamma_{i}\left(x_{0}\right)=\partial_{i} \Omega \cap Q\left(x_{0}\right), \quad i \in \mathfrak{J},
$$

si ha

$$
\Gamma\left(x_{0}\right)=\bigcup_{i \in \mathfrak{J}} \Gamma_{i}\left(x_{0}\right)
$$

Nel seguito, se $z$ è un punto di $R^{n}$, porremo

$$
\rho^{\tau}\left(x_{0}\right) z=\left(\rho^{\tau_{1}}\left(x_{0}\right) z_{1}, \ldots, \rho^{\tau_{n}}\left(x_{0}\right) z_{n}\right)
$$

Per ogni sottoinsieme $\mathfrak{D}$ di $\overline{I\left(x_{0}\right)}$, indicheremo con $\mathfrak{D}^{*}$ l'insieme (dei 
punti $\xi$ ) trasformato di $\mathfrak{D}$ mediante la sostituzione

$$
x-x_{0}=\rho^{\tau}\left(x_{0}\right)\left(\xi-x_{0}\right)
$$

e, per ogni funzione $v(x)$ definita in $\mathscr{D}$, porremo

$$
v^{*}\left(x_{0}, \xi\right)=v\left[x_{0}+\rho^{\tau}\left(x_{0}\right)\left(\xi-x_{0}\right)\right]
$$

Osservazione 7.2. - Facendo uso dell' osservazione 7.1, si verifica facilmente $\left({ }^{9}\right)$ che, assegnati comnnque $i \in \mathfrak{I}$ e $x_{0} \in \Omega$ tali che $\Gamma_{i}\left(x_{0}\right) \neq \varnothing$, esistono un intero positivo $p_{i}$, indipendente da $x_{0}$, e un ricoprimento di $\Gamma_{i}^{*}\left(x_{0}\right)$ formato da $p_{i}$ aperti di $R^{n}: \Theta_{i 1}, \ldots, \Theta_{i p_{i}}$ tali che ad ogni $\Theta_{i k}$ si possa associare un omeomorfismo $\xi \rightarrow \varphi_{i k}(\xi)$ di classe $C^{\infty}$ di $\theta_{i k} \cap I^{*}\left(x_{0}\right)$ su $\Sigma^{(i)}$ in modo che siano soddisfatte le seguenti condizioni:

$\left.s_{1}\right) \varphi_{i k}$ trasforma $\mathcal{O}_{i k} \cap \Gamma_{i}^{*}\left(x_{0}\right)$ su $\sigma^{(i)}$;

$\left.s_{2}\right) \quad$ posto $z=\varphi_{i k}(\xi)$, riesce $\partial z_{h}^{(r)} / \partial \xi_{j}^{(s)}=0$ per $r \neq s$ e $\partial z_{h} / \partial \xi_{h}>0$;

$\left.s_{3}\right)$ i moduli di continuità delle funzioni $\varphi_{i k}\left(\xi_{j}\right)$ e $\varphi_{i k}^{-1}(z)$ ed $i$ moduli di continuità delle derivate delle componenti di $\varphi_{i k}(\xi)$ e $\varphi_{i k}^{-1}(z)$ sono indipendenti da $x_{0}$;

$s_{4}$ ) i massimi moduli delle funzioni $\rho^{\tau}\left(x_{0}\right) \varphi_{i k}(\xi)$ e $\rho^{\tau}\left(x_{0}\right) \varphi_{i k}^{-1}(z)$ ed i massimi moduli delle derivate delle componenti di $\varphi_{i k}(\xi)$ e $\varphi_{i k}^{-1}(z)$ sono limitati da una costante indipendente da $x_{0}$.

Indicheremo con $L_{s}^{P}(\Omega), s$ reale e $0<p \leq \infty$, lo spazio delle funzioni $u(x)$ tali che $\rho^{s} u \in L^{p}(\Omega)$, munito della norma

$$
|\boldsymbol{u}|_{p, s}=\left\|\rho^{s} u\right\|_{L p(0)},
$$

Vogliamo richiamare, per comodità, i seguenti tre lemmi, contenuti rispettivamente nel lemma 4.1, nel lemma 4.2 e nel corollario II di [35] e dove poniamo $d_{0}=d /(d-1)$ :

Lemma 7.3. - Siano $p>0$ e s due numeri reali. Per ogni tunzione $u(x)$ tale che $\rho^{s}\left(x_{0}\right)|u|_{p, I\left(x_{0}\right)} \in L^{p}(\Omega)$ si ha

(9) Si tenga anche conto dell'osservazione 1.1 di [34]. 


$$
\int_{0}\left(\rho^{s}\left(x_{0}\right)|u|_{\left.P, I\left(x_{0}\right)\right)}\right) d x_{0} \geq c d_{0}^{-|s| p}|u|_{p, s+|\tau| p}^{p}
$$

dove c è una costante positiva indipendente da $u, s$ e p.

LEMMA 7.4. - Siano $p>0$ e s due numeri reali. Per ogni funzione $u(x) \in L_{s+|\tau| p \mid p}^{p}(\Omega)$ si $h a$

$$
\int_{\mathbf{0}}\left(\rho^{s}\left(x_{0}\right)|\boldsymbol{u}|_{\left.p, I\left(x_{0}\right)\right)^{p}} d x_{0} \leq c d_{0}^{|s|_{p}}|\boldsymbol{u}|_{p, s+|\pi| / p}^{p}\right.
$$

dove c è una costante indipendente da $u, s$ e $p$.

Lemma 7.5. - Siano $p>1$ e s due numeri reali.

Per ogni distribuzione $u$ su $\Omega$ tale che $u \in L_{s}^{p}(\Omega), D_{x_{k}}^{m_{k}} u \in L_{s+m_{k} \tau_{k}}^{p}(\Omega)(k=1$, ..., n), per ogni n-pla di interi non negativi a tale che $\langle\alpha, q\rangle<m$ e per ogni $a \in[<\alpha, q>/ m, 1]$ sussiste la limitazione

$$
\left|D^{\alpha} u\right|_{p^{\prime}, s+<\alpha, \tau>+|\tau|\left(1 / p-1 / p^{\prime}\right)} \leq c\left[\left(\sum_{k=1}^{n}\left|D_{x_{k}}^{m_{k}} \boldsymbol{u}\right|_{p, s+m_{k} \tau_{k}}\right)^{\alpha} \cdot|\boldsymbol{u}|_{p, s}^{1-\alpha}+|\boldsymbol{u}|_{p, s}\right]
$$

dove $p^{\prime}$ è $i l$ numero definito nel lemma 7.1 e c è una costante indipendente da $u$.

Indicheremo con $W_{s}^{k,}(\Omega), k \in J(\mathfrak{O K}), p$ reale $\geq 1$ e $s$ reale, lo spazio delle distribuzioni $u$ su $\Omega$ tali che $D^{\alpha} u \in L_{s+<\alpha, \tau>-k}^{p}(\Omega)$ per $\langle\alpha, q>\leq k$, munito della norma

$$
\|\boldsymbol{u}\|_{W_{\mathrm{s}}^{k, p}(\boldsymbol{\alpha})}^{k}=\sum_{<\alpha, q>\leq k}\left|D^{x} \boldsymbol{u}\right|_{p, s+<\alpha, \tau>-k}
$$

Porremo $W_{s}^{k}(\Omega)=W_{s}^{k, 2}(\Omega)$.

Osserviamo che riesce $W_{s}^{0, p}(\Omega)=L_{s}^{p}(\Omega)$ e che per ogni $k \in J(9 R)$ e $<m$ si ha $W_{s}^{m, p}(\Omega) \subset W_{s+\gamma_{k \rightarrow m}}^{k, p}(\Omega)$ algebricamente e topologicamente.

Sussiste inoltre il seguente

LEMMa 7.6. - Siano assegnati $i$ numeri reali $s, k$ e $p$, di cui $k \in J(\mathscr{T C})$ e $<m e p>1$.

Per ogni $\beta>s+k-m$, l'iniezione di $W_{s}^{m, p}(\Omega)$ in $W_{\beta}^{k_{s} p}(\Omega)$ è completamente continua. 
Poggiando sul lemma 7.5, con ragionamenti analoghi a quelli tenuti per dimostrare il lemma $1.5 \mathrm{di}[33]$, si prova che la tesi è vera per $k=0$, e ciod che per ogni $\beta>s-m$ l'iniezione di $W_{s}^{m, p}(\Omega)$ in $L_{\beta}^{p}(\Omega)$ è completamente continua.

Supposto ora $k>0$, osserviamo che, ancora in conseguenza del lemma 7.5 , si ha per ogni $\varepsilon>0$ e per ogni $u \in W_{s}^{m, p}(\Omega)$

$$
\|u\|_{W_{\beta}^{k}}^{k, p}(\Omega) \leq \varepsilon\|u\|_{w_{s}^{m, p}(\Omega)}+c(\varepsilon)\|u\|_{L_{\beta-k}^{p}(\Omega)} .
$$

D'altra parte, siccome per ipotesi $\beta-k>s-m$, da quanto premesso si ha che l'iniezione di $W_{s}^{m, p}(\Omega)$ in $L_{\beta-k}^{p}(\Omega)$ è completamente continua, e quindi da ogni successione $\left\{u_{n}\right\}$ limitata in $W_{s}^{m, p}(\Omega)$ :

$$
\left\|u_{n}\right\|_{D_{s}^{m, p}(\Omega)}^{m} \leq M \quad \forall n
$$

se ne può estrarre una convergente in $L_{\beta-k}^{p}(\Omega)$.

Osservando allora che, in conseguenza della (7.12), la relazione

$$
\left\|u_{i}-u_{j}\right\|_{\beta-k}^{p}(\Omega) \leq \frac{\varepsilon}{c(\varepsilon)}
$$

impliea che si ha

$$
\left\|u_{i}-u_{j}\right\|_{W_{\beta}^{k}}^{k, P}(\Omega) \leq(2 M+1) \varepsilon,
$$

si ottiene evidentemente la tesi.

Per ogni $\varepsilon>0$ sufficientemente piccolo, indicheremo con $\delta_{\varepsilon}(x)$ una funzione di classe $C^{\infty}(\bar{\Omega})$ e tale che

$$
\begin{aligned}
& \delta_{\varepsilon(x)}\left\{\begin{array}{l}
=1 \text { se } \rho(x) \geq 2 \varepsilon \\
=0 \text { se } \rho(x) \leq \varepsilon,
\end{array}\right. \\
& \left|D^{\alpha \delta_{\varepsilon}}(x)\right| \leq c_{\alpha \alpha P^{-|\alpha|}(x)} \quad \forall x \in \bar{\Omega},
\end{aligned}
$$

dove $c_{\alpha}$ è una costante dipendente solo da $\Omega, S$ e $\alpha$.

Lемма 7.7. - Per ogni $u \in W_{s}^{k, p}(\Omega), k \in J(\Re)$, p reale $\geq 1$ e s reale, si ha

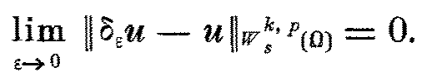


Infatti, osserviamo che riesce

$$
\begin{aligned}
\| \delta_{\varepsilon} u- & u \|_{s}^{k, p}(\Omega)=\sum_{<\alpha, q>S_{k}}^{\Sigma}\left|D^{\alpha}\left[\left(1-\delta_{\varepsilon}\right) u\right]\right|_{p, s+<\alpha, \tau>-k} \\
& \leq c_{1}\left(\sum_{<\alpha, q>\leq k}\left|\left(1-\delta_{\varepsilon}\right) D^{\alpha} u\right|_{p, s+<\alpha, \tau>-k}\right. \\
& \left.+\sum_{<\alpha, q>\leq k} \sum_{\beta<\alpha}\left|D^{\alpha-\beta \delta_{\varepsilon}} \cdot D^{\beta} u\right|_{p, s+<\alpha, \tau>-k}\right),
\end{aligned}
$$

dove $c_{1}$ è una costante indipendente da $u$ e $\varepsilon$.

Tenuto conto delle (7.13), (7.14) ed osservando che per $\beta \leq \alpha$ riesce

$$
s+\langle\alpha, \tau>-k-|\alpha-\beta| \geq s+\langle\beta, \tau>-k,
$$

dalla precedente relazione con note considerazioni si deduce la tesi.

Dimostriamo inoltre il seguente

LEMMA 7.8. - Qualunque siano $k \in J(\mathfrak{O K}), p$ reale $\geq 1$ e s reale, $C^{\infty}(\bar{\Omega}) \grave{e}$ denso in $W_{s}^{k, p}(\Omega)$.

Infatti, sia $u$ una funzione di classe $W_{s}^{k, p}(\Omega)$.

Cominciamo con l'osservare che, tenuto conto della (7.14), si ha

$$
\begin{gathered}
\left\|\delta_{\varepsilon} u\right\|_{D_{s}^{k, p}(0)}^{k, p} c_{1} \sum_{<\alpha, q>\leq k} \sum_{\beta \leq \alpha}^{\Sigma}\left|D^{x-\beta \delta_{\varepsilon}} \cdot D^{\beta} \boldsymbol{u}\right|_{p, s+<\alpha, \tau>-k} \\
\quad \leq c_{2} \sum_{<\alpha, q>\leq \leq k} \sum_{\beta \leq \alpha}\left|D^{\beta} \boldsymbol{u}\right|_{P, s+<\alpha, \tau>-k-|\alpha-\beta|},
\end{gathered}
$$

dove $c_{1}$ e $c_{2}$ sono due costanti indipendenti da $u$ e $\varepsilon$.

Tenuto quindi conto della $(7.16)$, si deduce che esiste una costante $c$ indipendente da $u \in \varepsilon$ tale che si ha

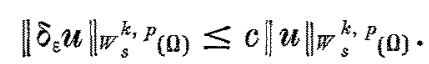

Inoltre, siccome $\delta_{\mathrm{s}} u \in \grave{W}^{k, p}(\Omega)$, dal lemma 7.2 segue che, per ogni $\varepsilon$, esiste una successione $\left\{u_{n}^{(\varepsilon)}\right\}$ di funzioni di classe $C^{\infty}(\bar{\Omega})$ convergente a $\delta_{\varepsilon} u$ in $W^{k, p}(\Omega)$. riesce

D'altra parte, tenendo presente la definizione di $\delta_{\varepsilon}(x)$, è subito visto che 


$$
\begin{gathered}
\left\|\delta_{\varepsilon} u_{n}^{(\varepsilon)}-\delta_{\varepsilon}^{2} u\right\|_{w^{k}}^{k, p}(0) \leq c(\varepsilon)\left\|\delta_{\varepsilon} u_{n}^{(\varepsilon)}-\delta_{\varepsilon}^{2} u\right\|_{W^{k}}^{k, p}(0) \\
\leq c^{\prime}(\varepsilon)\left\|u_{n}^{(\varepsilon)}-\delta_{\varepsilon} u\right\|_{W^{k, p}}^{k(0)}
\end{gathered}
$$

dove $c(\varepsilon)$ e $c^{\prime}(\varepsilon)$ sono due costanti indipendenti da $u$ e $n$.

Consegue che, fissato $\varepsilon$, la successione $\left\{\delta_{\varepsilon} u_{n}^{(\varepsilon)}\right\}$ converge a $\delta_{\varepsilon}^{2} u$ in $W_{s}^{k, p}(\Omega)$, e quindi esiste $n_{\varepsilon}$ tale che si ha

$$
\left\|\delta_{\varepsilon} u_{n_{\varepsilon}}^{(\xi)}-\grave{\delta}_{\varepsilon}^{2} u\right\| w_{s}^{k, p}(0) \leq \varepsilon
$$

Posto $u_{\varepsilon}=\delta_{\varepsilon} u_{n_{\varepsilon}}^{(\varepsilon)}$, si ha per le $(7.17)$ e $(7.18)$

$$
\begin{aligned}
& \left\|u_{\varepsilon}-u\right\|_{W_{s}^{k, p}(\Omega)}^{k,} \leq\left\|u_{\varepsilon}-\delta_{\varepsilon}^{2} u\right\| W_{s}^{k, p}(\Omega) \\
& +\left\|\delta_{\varepsilon}^{2} u-\delta_{\varepsilon} u\right\|_{W_{s}^{k, P}(\Omega)}+\left\|\delta_{\varepsilon} u-u\right\|_{W_{s}^{k, P}(\Omega)} \\
& \leq \varepsilon+(1+c)\left\|\delta_{\varepsilon} u-u\right\|_{p_{s}^{k, p}}^{k,(\Omega)} \text {. }
\end{aligned}
$$

Da quest'ultima relazione e dal lemma 7.7 segue

$$
\lim _{\varepsilon \rightarrow 0}\left\|\boldsymbol{u}_{\varepsilon}-u\right\|_{\mathbb{W}_{s}^{k, p}(\Omega)}=0
$$

e quindi si ha la tesi.

Richiamiamo anche il seguente risultato contenuto nel teorema 2.3 del cap. 6 di J. NEC̈AS [26]:

Lemma 7.9. - Siano: 9 un insieme aperto, limitato ed a frontiera «local. mente lipschitziana» $\left({ }^{10}\right)$ di uno spazio reale euclideo $R^{N}$; $\sigma(x)$ una funzione di classe $C^{\circ}(\bar{D})$, positiva in $\mathfrak{D}$ e tale che si abbia in coordinate locali $\left(x_{r}^{\prime}, x_{r N}\right)\left({ }^{11}\right)$

$$
c_{1} \sigma(x) \leq x_{r N}-a_{r}\left(x_{r}^{\prime}\right)+\chi_{r}\left(x_{r}^{\prime}\right) \leq c_{2} \sigma(x)
$$

(10) Cioè $Q \in \mathscr{V}^{(0), 1}$ nel senso di J. NEÕAs [26] (cfr. in [26] il n. 1.3 del cap. 1 e il n. 1.1 del cap. 2).

(ii) Facciamo uso delle notazioni di J. NECAs [26] (cfr, i riferimenti citati nella nota (10) e la (2.1) del cap. 6 di [26]). 
dove $c_{1}$ e $c_{2}$ sono due costanti positive, $\chi_{r}\left(x_{r}^{\prime}\right)$ è una funzione detinita in $\Delta_{r} e$ tale che $0 \leq \chi_{r}\left(x_{r}^{\prime}\right) \leq$ cost.

Allora, fissati $i$ numeri reali $p>1$ e $s_{0}<1-1 / p$, per ogni $s \leq s_{0}$ e per ogni $u \in C^{1}(\overline{\mathscr{D}})$ tale che $u=0$ su $\partial \mathscr{D}, D_{x_{k}} u \in L_{s}^{p}(\mathfrak{D})(k=1, \ldots, N)$, sussiste la limitazione

$$
\int_{\mathscr{D}}\left|\sigma^{s-1} u\right|^{p} d x \leq c \sum_{h=1}^{N} \int_{\mathscr{D}}\left|\sigma^{s} D_{x_{k}} u\right|^{p} d x,
$$

dove $c$ è una costante indipendente da $u$ e s.

Osservazione 7.3. - Si verifica facilmente che, se $\Omega$ ha frontiera «localmente lipschitziana», la fanzione $\sigma(x)=\rho(x)$ verifica le ipotesi del lemma 7.9 con $\mathfrak{D}=\Omega$ e $\chi_{r}\left(x_{r}^{\prime}\right)=\rho(\dot{x})$, dove $\dot{x}=\left(x_{r}^{\prime}, a_{r}\left(x_{r}^{\prime}\right)\right)$. Inoltre se $t=2, S^{(2)}=\emptyset e$ $\Omega^{(1)}$ ha frontiera «localmente lipschitziana», fissato $y \in \Omega^{(2)}$, la funzione $\sigma(x)=\rho(x, y)$ definita in $\overline{\Omega^{(1)}}$ verifica le ipotesi del lemma 7.9 con $\mathscr{D}=\Omega^{(1)}$, $\chi_{r}\left(x_{r}^{\prime}\right)=\sigma(\dot{x})\left(\right.$ dove $\left.\dot{x}=\left(x_{r}^{\prime}, a_{r}\left(x_{r}^{\prime}\right)\right)\right)$ e con le costanti indipendente da $y$.

\section{8. - Problemi quasi-ellittici: ipotesi e risultati.}

Assegnamo in $\Omega$ un operatore differenziale lineare della forma

$$
A=A(x, D)=\sum_{<\mu, q>\leq m}^{\Sigma} a_{\mu}(x) D^{\mu}
$$

di cui indicheremo con $A_{0}$ la parte principale:

$$
A_{0}=A_{0}(x, D)=\sum_{\langle\mu, q>=m} a_{\mu}(x) D^{\mu} .
$$

Supporremo che siano verificate le seguenti ipotesi:

$\left.\mathscr{H}_{1}\right)$ Esiste un numero reale $\chi$ tale che le funzioni $\rho^{\chi-\langle\mu, r>}(x) a_{\mu}(x)$ siano continue in $\bar{\Omega}$ per $<\mu, q>=m$, misurabili e limitate in $\Omega$ per $<\mu, q><m$; inoltre è soddisfatta la seguente condizione di quasi-ellitticità:

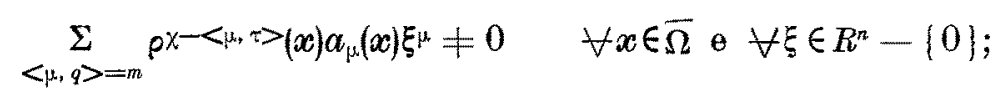

$\left.\mathscr{H}_{2}\right)$ Per ogni $i \in \mathfrak{J}, A$ e propriamente quasi-ellittico di tipo $v_{i}$ su $\partial_{i} \Omega$, 
cios per $x \in \partial_{i} \Omega$, per ogni vettore reale $\xi \neq 0$ tangente a $\gtrsim_{i} \Omega$ in $x$ e per ogni vettore reale $\xi^{\prime} \neq 0$ normale a $\partial_{i} \Omega$ in $x$, il polinomio $A_{0}\left(x, \xi+z \xi^{\prime}\right)$, nella variabile complessa $z$, ha esattamente $v_{i}$ radici con parte immaginaria positiva $\left({ }^{12}\right)$.

Se $w^{(i)}(x)$ è una funzione definita su $\partial_{i} \Omega$ e $\theta_{i k}$ è uno degli omeomorfismi che intervengono nella definizione 7.1, porremo per ogni $y \in \sigma^{(i)}$

$$
w^{(i, h)}(y)=w^{(i)}\left[\theta_{i k}^{-1}(y)\right]
$$

Assegnamo inoltre, per ogni $i \in \mathfrak{O}$, un sistema $\left\{B_{i j}\right\}_{j=1}^{v_{i}}$ di $v_{i}$ operatori differenziali lineari di frontiera definiti su $\partial_{i} \Omega$ e della forma

$$
B_{i j}=B_{i j}(x, D)=\sum_{\left\langle\mu, q>\leq p_{i j} q^{(i)}\right.} b_{j j^{\mu}}^{(i)}(x) D^{\mu}, \quad p_{i j} \leq m^{(i)}-1
$$

di cui indicheremo con $B_{0 i j}$ la parte principale:

$$
B_{0 i j}=B_{0 i j}(x, D)=\underset{<\mu, q>=p_{i j} g^{(i)}}{\Sigma} b_{j \mu}^{(i)}(x) D^{\mu}
$$

Supporremo che siano verificate le seguenti ipotesi:

$\left.\mathscr{H}_{3}\right)$ I coefficienti $b_{j \beta}^{(i)}(x)$ sono di classe $C^{\infty}\left(\partial_{i} \Omega\right)$; inoltre esistono dei numeri reali $\gamma_{i}(i \in \mathfrak{J})$ tali che le funzioni $\rho_{i}+p_{i} \tau^{\tau^{(i)}}-<\mu . r(x) b_{j \mu}^{(i)}(x) \in C^{0}\left(\overline{\left.\partial_{i} \Omega\right)}\right.$ per $<\mu, q>=p_{i j} q^{(i)}$ e tali che si abbia per $<\mu, q>\leq p_{i j} q^{(i)}$, per ogni $y \in \sigma^{(i)}$ e per ogni $\beta$

$$
\left|\left(\rho^{(i, k)}(y)\right)_{i}+p_{i, \tau^{\tau}}^{(i)}+<\beta, \tau>-<\mu, \tau>D_{y}^{\beta} b_{j p}^{(i, k)}(y)\right| \leq M_{\beta},
$$

dove $M_{\beta}$ è una costante indipendente da $y$;

$\left.\mathscr{H}_{4}\right)$ Per ogni $i \in \mathfrak{J}$, gli operatori $B_{i 1}, \ldots, B_{i_{i}}$ soddisfano la condizione complementare rispetto ad $A$ su $\partial_{i} \Omega$, cioè per ogni $x \in \partial_{i} \Omega$, per ogni vettore reale $\xi \neq 0$ tangente a $\partial_{i} \Omega$ in $x$ e per ogni vettore reale $\xi^{\prime} \neq 0$ normale a $\partial_{i} \Omega$ in $x$, i polinomi $B_{0 i 1}\left(x, \xi+z \xi^{\prime}\right), \ldots, B_{0 i v_{i}}\left(x, \xi+z \xi^{\prime}\right)$, nella variabile complessa $z$, sono linearmente indipendenti modulo il polinomio $\prod_{k=1}^{v_{i}}\left(z-z_{k}^{+}\left(x, \xi, \xi^{\prime}\right)\right)$, dove

(12) Osserviamo che, nel caso $n>2$, da quanto rilevato nell'osservazione 5.1 segue che, se $\partial_{i} \Omega$ è un insieme connesso, ogni operatore quasi-ellitico su $\partial_{i} \mathrm{Q}$ è propriamente quasiellittico su $\partial 2$. 
le $z_{k}^{+}\left(x, \xi, \xi^{\prime}\right)$ sono le radici con parte immaginaria positiva di $A_{0}\left(x, \xi+z \xi^{\prime}\right)$.

Nel seguito, assegnato comunque $x_{0} \in \Omega$, porremo

$$
\tilde{A}=\tilde{A}\left(x_{0}, \xi, D\right)=\sum_{<\mu, q>\leq m} \rho^{x-<\mu, \tau>\left(x_{0}\right) a_{\mu}^{*}\left(x_{0}, \xi\right) D^{\mu}}
$$

$e$, se $\Gamma_{i}\left(x_{0}\right) \neq \emptyset$,

$$
\tilde{B}_{i j}=\tilde{B}_{i j}\left(x_{0}, \xi, D\right)=\sum_{<\mu, q>\leq p_{i j} q^{(i)}} \rho^{r_{i}+p_{i j} \tau^{(i)}-<\mu, \tau>}\left(x_{0}\right) b_{j \mu}^{(i) *}\left(x_{0}, \xi\right) D^{\mu}
$$

Osservazione 8.1. - Sia $\sigma(x)$ una fanzione di classe $C^{0,1}(\bar{\Omega})$ e tale che si abbia

$$
c_{1} \rho(x) \leq \sigma(x) \leq c_{2} \rho(x) \quad \forall x \in \bar{\Omega},
$$

dove $c_{1}$ e $\mathbf{c}^{2}$ sono dne costanti positive indipendenti da $x$.

Dalla lipschitzianita di $\sigma(x)$ e dalle (7.3), (8.10) segue che per ogni nnmero reale $s$ la funzione $\left(\sigma^{*}\left(x_{0}, \xi\right) / \rho\left(x_{0}\right)\right)^{s}$ risulta continua rispetto a $\xi$ in $\overline{7^{*}\left(x_{0}\right)}$ uniformemente al variare di $x_{0}$ in $\Omega$.

Ossmrvazione 8.2. - Tenendo conto dell'osservazione 8.1 ed osservando che si può scrivere

$$
\begin{gathered}
\rho x-<\mu, \tau>\left(x_{0}\right)=\left(\frac{\rho^{*}\left(x_{0}, \xi\right)}{\rho\left(x_{0}\right)}\right)^{<\mu, \tau>-\psi} \cdot \rho^{* \chi}-<\mu, \tau>\left(x_{0}, \xi\right), \\
\rho Y_{i}+p_{i j} \tau^{(i)}-<\mu, \tau>\left(x_{0}\right)=\left(\frac{\rho^{*}\left(x_{0}, \xi\right)}{\rho\left(x_{0}\right)}\right)^{<\mu, \tau>-\gamma_{i}-p_{i j} \tau^{(i)}} \cdot \rho^{* \gamma_{i}+p_{i j} \tau} \tau^{(i)}-<\mu_{*} \tau>\left(x_{0}, \xi\right),
\end{gathered}
$$

si verifica facilmente che, se sono soddisfatte le ipotesi $\left.\mathfrak{H}_{1}\right)-\mathscr{H}_{4}$, fissato uno degli omeomorfismi $\varphi_{i k}$ definiti nell'osservazione 7.2, mediante $\varphi_{i k}$ l'operatore $\tilde{A}$ e il sistema $\left\{\tilde{B}_{i j}\right\}_{j=1}^{v_{i}}$ vengono trasformati in un operatore $\AA$ della forma

$$
\stackrel{\leftrightarrow}{A}=\sum_{<\mu, q>\leq m}^{\Sigma} \stackrel{\circ}{a_{\mu}}\left(x_{0}, z\right) D^{\mu}
$$

e in un sistema $\left\{\mathscr{B}_{i j}\right\}_{j=1}^{v_{i}}$ di operatori della forma

$$
\stackrel{\mathscr{B}}{i j}_{i j}=\underset{<\mu, q>\leq p_{i j} q^{(i)}}{\Sigma} \tilde{b}_{j(j)}^{(i)}\left(x_{0}, z^{\prime}\right) D^{\mu}
$$


dove $z^{\prime}=\left(z_{1}, \ldots, z_{n-1}\right)$, soddisfacenti le seguenti condizioni:

$\left.t_{1}\right)$ i coefficienti $\stackrel{\circ}{a}_{\mu}\left(x_{0}, z\right)$ hanno moduli limitati in $\Sigma^{(i)}$ da una costante indipendente da $x_{0}$; inoltre $\mathrm{i}$ coefficienti $\stackrel{a}{a}_{\mu}\left(x_{0}, z\right)$ per $\langle\mu, q\rangle=m$ sono continui in $\overline{\Sigma^{(i)}}$ ed hanno moduli di continuità indipendenti da $x_{0}$;

$\left.t_{2}\right)$ i coefficienti $b_{j \mu}^{(i)}\left(x_{0}, z\right)$ sono di classe $C^{\infty} \overline{\left(\sigma^{(i)}\right)}$ ed i loro massimi moduli e quelli delle loro derivate sono limitati da una costante indipendente da $x_{0}$; inoltre i coefficienti $b_{j \mu}^{(i)}\left(x_{0}, z\right)$ per $\langle\mu, q\rangle=p_{i j} q^{(i)}$ hanno moduli di continuità indipendenti da $x_{0}$;

$\left.t_{3}\right)$ è verificata la condizione di quasi-ellitticità :

$$
\left|\sum_{<\mu, q>=m} \dot{a}_{i \mu}\left(x_{0}, z\right) \xi^{\mu}\right| \geq \alpha_{0} \sum_{i=1}^{n}\left|\xi_{i}\right|_{i}^{m_{i}}
$$

dove $\alpha_{0}$ è una costante positiva indipendente da $z, \xi$ e $x_{0}$;

$\left.t_{4}\right) \AA$ è propriamente quasi-ellittico di tipo $v_{i}$ su $\overline{\sigma^{(i)}}$ e gli operatori $\vec{B}_{i 1}, \ldots,{\stackrel{B}{i y_{i}}}_{i}$ verificano la condizione complementare rispetto ad $\dot{A}$ su $\overline{\sigma^{(i)}}$.

Sussiste il seguente teorema di regolarizzazione:

Teorema 8.1. - Siano verificate le ipotesi $\left.\left.\left.a_{0}\right), \mathscr{H}_{1}\right)-\mathscr{H}_{4}\right)$ e sia $s$ un assegnato numero reale.

Per ogni funzione $u \in L_{s(\Omega)}^{2}$ e tale che

$$
u \in W^{m}(T) \quad \forall \text { compatto } T \subset \bar{\Omega}-S,
$$

$$
A u \in L_{s+\chi}^{2}(\Omega),
$$

$$
B_{i j} u=0 s u \partial_{i} \Omega\left({ }^{13}\right), \quad j=1, \ldots, v_{i}, i \in \mathfrak{d} .
$$

si ha che $u \in W_{s+m}^{m}(\Omega)$ e sussiste la limitazione

$$
\|u\|_{W_{s+m}^{m}(\Omega)} \leq c\left(\|A u\|_{L_{s+\chi^{2}}^{2}(0)}+\|u\|_{L_{s}^{2}(0)}\right),
$$

dove c è una costante indipendente da $u$.

(13) Qui e nel seguito l'annullarsi dei dati su $\partial_{i} Q$ va inteso localmente nel senso del teorema di tracce enunciato al n. 1 (cfr. il lemma 1.7). 
La dimostrazione di questo teorema sarà data al successivo n. 9.

Consideriamo ora il problema

$$
\begin{aligned}
& u \in W_{s}^{m}(\Omega), \quad s \text { reale, } \\
& A u+\lambda \sigma-\chi_{u}=f \text { in } \Omega, \quad f \in L_{s+\chi-m}^{2}(\Omega), \\
& B_{i j} u=0 \text { su } \partial_{i} \Omega, \quad j=1, \ldots, \nu_{i}, \quad i \in \mathfrak{J},
\end{aligned}
$$

dove $\lambda \dot{\theta}$ un numero complesso, $\chi$ è il numero definito nell'ipotesi $\left.\mathscr{H}_{1}\right)$ e $\sigma(x)$ $\dot{\theta}$ una funzione di classe $C^{0,1}(\bar{\Omega})$ e soddisfacente la (8.10).

Associamo ad $x$ il punto $(x, y)$, dove $y$ è una variabile reale, e poniamo $D_{x}=D, D_{y}=-i \partial / \partial y$.

Inoltre associamo ad $A\left(x, D_{x}\right)=A(x, D)$ l'operatore

$$
\mathfrak{S}_{\theta, l}=\mathcal{S}_{\theta, l}\left(x, D_{x}, D_{y}\right)=A\left(x, D_{x}\right)+e^{i \theta} D_{y}^{l},
$$

dove $\theta$ è un numero reale e $l$ è un intero positivo.

Stabiliremo un teorema di unicità per il problema (8.15)-(8.17), suppo. nendo che siano verificate le ipotesi del teorema 8.1 di regolarizzazione e la seguente ulteriore ipotesi:

$\left.\mathscr{H}_{5}\right)$ Esistono $\theta$ e $l$ tali che sia verificata la seguente condizione di quasi-ellitticità :

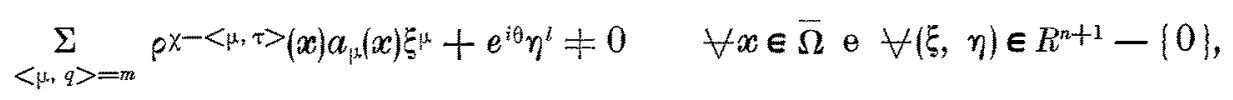

e gli operatori $B_{i 1}\left(x, D_{x}\right), \ldots, B_{i v_{i}}\left(x, D_{x}\right)$ verifichino la condizione complementare rispetto a $\mathcal{L}_{\theta, i}\left(x, D_{x}, D_{y}\right)$ su $\partial_{i} \Omega \times R^{1}\left({ }^{14}\right)$.

Teorema 8.2. - Siano verificate le ipotesi $\left.a_{0}\right)$ e $\left.\mathscr{H}_{1}\right)-\mathcal{H}_{5}$ ).

Allora, fissato $s$, esiste una costante $\gamma_{0}>0$, indipendente da $s$, tale che per ogni $\lambda$, con $|\lambda| \geq \gamma_{0} d_{0}^{2 l|s|}\left({ }^{15}\right)$ e arg $\lambda=\theta$, una eventuale soluzione $u$ del problema (8.15)-(8.17) soddisfa la limitazione

$$
\|u\|_{\mathbb{P}_{s}^{m}(0)} \leq c d_{0}^{2|s|}\|f\|_{L_{s+\chi}^{2}-m}(\Omega)
$$

dove $c$ è una costante indipendente da $u, f, s e \lambda$.

(14) Osserviamo che, essendo $n+1>2$, in conseguenza dell'ipotesi $\mathfrak{H}_{2}$ ), dell'ipotesi di quasi-ellitticità di $\varrho_{\theta, l}$ e di quanto rilevato nell'osservazione 5.1 , si ha che $\varrho_{\theta, l}\left(x, D_{x}, D_{y}\right)$ riesce, per ogni $i \in \mathfrak{g}$, propriamente quasi-ellittico di tipo $v_{i}$ su $\partial_{i} \Omega \times R^{1}$.

(15) $d_{0}$ è la costante che figura nei lemmi 7.3 e 7.4 . 
La dimostrazione di questo teorema di unieità sarà data al n. 10.

Volendo ora passare ad enunciare un teorema di esistenza per il proble. ma (8.15)-(8.17), poniamo la seguente

Definizione 8.1. - Fissato $i \in \mathfrak{g}$, diremo che un sistema $\left\{B_{j}\right\}_{j=1}^{r}$ di $r$ operatori differenziali lineari di frontiera della forma

$$
B_{j}=\underset{<\mu, q>\leq p_{j}}{\Sigma} b_{j \mu}(x) D^{\mu}
$$

e con i coefficienti $b_{j \mu}(x)$, per $\langle\mu, q\rangle=p_{j}$, definiti su una porzione $\Gamma$ di $\overline{\partial_{i} \Omega}$, è normale su $\Gamma$, se si ha $p_{j} \neq p_{k}$ per $j \neq k$ e

$$
\sum_{<\mu, q>=p_{j}} b_{j \mu}(x) \xi^{\mu-} \neq 0
$$

per ogni $x \in \Gamma$ e per ogni vettore $\xi \in R^{n}-\{0\}$ normale a $\Gamma$ in $x$.

Agginngiamo allora alle ipotesi del teorema 8.2 di unicità le seguenti ipotesi :

$\left.\mathscr{H}_{6}\right)$ I coefficienti $a_{\mu}(x) \in C^{\infty}(\bar{\Omega}-S)$ e le derivate $D^{\alpha} a_{\mu}(x) \in$ $\boldsymbol{\epsilon} L_{\chi+<\alpha, \tau>-<\mu, \tau>}^{\infty}(\Omega)$;

$\left.\mathcal{H}_{7}\right)$ Per ogni $i \in \mathfrak{J}$, il sistema $\left\{B_{i 1}^{\left(\gamma_{i}, \tau\right)}, \ldots, B_{\substack{i_{i} \\ i}}^{\left(\gamma_{i}, \tau\right)}\right\}$, dove

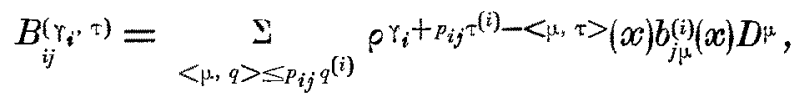

è normale su $\overline{\partial_{i} \Omega}$;

$\left.\mathscr{H}_{8}\right)$ Esiste una successione $\left\{\sigma_{k}(x)\right\}$ di funzioni di classe $C^{\infty}(\bar{\Omega}-S) \cap$ $\cap C^{0.1}(\bar{\Omega})$ e tali che

$$
\begin{array}{ll}
c_{1} \rho(x) \leq \sigma_{k}(x) \leq c_{2} \rho(x) & \forall x \in \bar{\Omega}, \\
\left|\sigma_{k}\left(x^{\prime}\right)-\sigma_{k}\left(x^{\prime \prime}\right)\right| \leq c_{3}\left|x^{\prime}-x^{\prime \prime}\right| & \forall x^{\prime}, x^{\prime \prime} \in \bar{\Omega},
\end{array}
$$

con $c_{1}, c_{2}$ e $c_{3}$ costanti positive indipendenti da $k$, convergente a $\sigma(x)$ in $C^{0,1}(\bar{\Omega})$. 
Teorema 8.3. - Siano verificate le ipotesi $\left.a_{0}\right)$ e $\left.\left.\mathscr{H}_{\mathrm{1}}\right)-\mathscr{H}_{8}\right)$.

Allora, fissato $s$, esiste una costante $\lambda_{0}>0$ tale che per ogni $\lambda$, con $|\lambda| \geq \lambda_{0}$ e $\arg \lambda=0$, e per ogni $f \in L_{s+\chi-m}^{2}(\Omega)$ il problema $(8.15)-(8.17)$ è univo. camente risolubile.

Questo teorema di esistenza sarà dimostrato al n. 11 .

Supponiamo in particolare che $A$ verifichi la seguente condizione di forte quasi-ellitticitòt:

$$
R e \sum_{<\mu, q>=m} \rho^{x-<\mu, r>}(x) a_{\mu}(x) \xi^{\mu}>0 \quad \forall x \in \bar{\Omega} \text { e } \forall \xi \in R^{n}-|0|
$$

In tal caso, da noti risultati (efr. G. C. BARozzI [5]) segue che gli interi $m_{1}, \ldots, m_{n}$ sono pari e che è soddisfatta la condizione $\left.\mathscr{H}_{2}\right)$ di quasi-ellitticità propria con $v^{(i)}=m^{(i)} / 2$.

Considerando allora il problema di DiRIchlem

$$
\begin{aligned}
& u \in W_{s}^{m}(\Omega), \quad s \text { reale, } \\
& A u+\lambda \sigma^{-\chi} u=f \text { in } \Omega, f \in L_{s+\chi}^{2}-m(\Omega), \\
& \frac{\partial^{j} u}{\partial \boldsymbol{n}_{i}^{j}}=0 \text { su } \partial_{i} \Omega, \quad j=0, \ldots, \frac{m^{(i)}}{2}-1, i \in \mathfrak{J},
\end{aligned}
$$

dove $n_{i}$ denota la normale a $\partial_{i} \Omega$ orientata, ad es., verso l'interno di $\Omega$, si ha:

Conoll ario 8.1. - Supponiamo che $i$ coefficienti $a_{\mu}(x)$ verifichino le ipotesi $\left.\left.\mathscr{H}_{1}\right), \mathfrak{H}_{6}\right)$ e che siano soddisfatte la condisione (8.23) di quasi-ellitticità forte $e$ le ipotesi $\left.\left.a_{0}\right), \mathfrak{H}_{8}\right)$.

Allora, fissato $s$, esiste una costante $\lambda_{0}>0$ tale che per ogni $\lambda$, con Re $\lambda \geq \lambda_{0}$, e per ogni $f \in L_{s+\chi}^{2}-m(\Omega)$ il problema (8.24) è univocamente risolubile.

La tesi consegue dal teorema 8.3 osservando che, se è verificata la condizione $(8.23)$ di forte quasi-ellitticità e $B_{i j}=\left(\partial / \hat{\partial} n_{i}\right)^{j}$, l'ipotesi $\left.\mathfrak{I}_{5}\right)$ è soddisfatta per $l=m$ e per ogni $\theta \in]-\pi / 2, \pi / 2[$.

\section{9. - Dimostrazione del teorema 8.1.}

Premettiamo un lemma.

Per ogni numero reale $r>0$, porremo 


$$
\begin{aligned}
& \Gamma_{r}=\left\{x=\left(x^{(1)}, \ldots, x^{(i)}\right) \in R^{n}|| x^{(i)} \mid<r, i=1, \ldots, t\right\}, \\
& \boldsymbol{\Sigma}_{r}=\left\{x \in \Gamma_{r} \mid x_{n} \geq 0\right\}, \\
& \sigma_{r}=\left\{x \in \Gamma_{r} \mid x_{n}=0\right\},
\end{aligned}
$$

e indicheremo con $Q_{r}$ l'uno o l'altro dei due insiemi $\Gamma_{r}$ e $\Sigma_{r}$.

Fissato $r_{0}>0$, assegnamo in $Q_{r_{0}}$ un operatore differenziale lineare della forma

$$
A=A(x, D)=\underset{<\mu, q>\leq m}{\Sigma} a_{\mu}(x) D^{\mu}
$$

supponendo che siano verificate le seguenti ipotesi:

$\left.\mathscr{G}_{1}\right)$ I coefficienti $a_{\mu}(x)$ sono continui in $\bar{Q}_{r_{0}}$ per $\langle\mu, q\rangle=m$, misurabili e limitati in $Q_{r_{0}}$ per $<\mu, q><m$;

$\left.\mathscr{G}_{2}\right) \quad A$ è quasi-ellittico in $\bar{Q}_{r_{0}}$ e, nel caso $Q_{r_{0}}=\Sigma_{r_{0}}$, propriamente quasiellitico di tipo $\vee$ su $\bar{\sigma}_{r_{0}}$.

Inoltre, se $Q_{r_{0}}=\Sigma_{r_{0}}$, assegnamo su $\sigma_{r_{0}}$ un sistema $\left\{B_{j}\right\}_{j=1}^{\nu}$ di $\vee$ operatori differenziali lineari di frontiera della forma $(8.20)$ e soddisfacenti le seguenti ipotesi :

$\left.\mathscr{G}_{3}\right)$ Risulta $p_{j} \leq m-q_{n}$ e i coefficienti $b_{j \mu}(x) \in C^{\infty}\left(\bar{\sigma}_{r_{0}}\right)$;

$\left.\mathcal{G}_{4}\right) B_{1}, \ldots, B_{v}$ verificano la condizione complementare rispetto ad $A$ su $\bar{\sigma}_{r_{0}}$.

Indicheremo con $V\left(\Sigma_{r_{0}}\right)$ la classe delle funzioni $u \in W^{m}\left(\Sigma_{r_{0}}\right)$ e tali che

$$
B_{j} u=0 \quad \text { sn } \quad \sigma_{r_{0}}, \quad j=1, \ldots, v,
$$

$\theta$ con $V\left(\Gamma_{r_{0}}\right)$ lo spazio $W^{m}\left(\Gamma_{r_{0}}\right)$

Lemma 9.1. - Nelle ipotesi sopra poste, per ogni $u \in V\left(Q_{r_{0}}\right)$ sussiste la limitazione

$$
\underset{<\alpha, q>=m}{\Sigma}\left|D^{\alpha} \boldsymbol{u}\right|_{2, Q_{r}} \leq c\left(|A \boldsymbol{u}|_{2, Q_{r_{0}}}+|\boldsymbol{u}|_{2, Q_{T_{0}}}\right)
$$

dove $r<r_{0}$ e dove c è una costante indipendente $d a u$. 
Infatti, assegnati tre numeri positivi $r<r^{\prime}<r^{\prime \prime}<r_{0}$, con $r^{\prime}-r=r^{\prime \prime}-r^{\prime}<1$, consideriamo due funzioni $\zeta(x)$ e $\varphi(x)$ di classe $C_{0}^{\infty}\left(R^{n}\right)$, tali che

$$
\zeta(x)\left\{\begin{array} { l l l } 
{ = 1 } & { \text { se } } & { x \in \Gamma _ { r } } \\
{ = 0 } & { \text { se } } & { x \in R ^ { n } - \Gamma _ { r ^ { \prime } } , }
\end{array} \quad \varphi ( x ) \left\{\begin{array}{lll}
=1 & \text { se } & x \in \Gamma_{r^{\prime}} \\
=0 & \text { se } & x \in R^{n}-\Gamma_{r^{\prime \prime}}
\end{array}\right.\right.
$$

e tali inoltre che si abbia

$$
\left|D^{\alpha} \zeta(x)\right| \leq M_{\alpha}\left(r^{\prime}-r\right)^{-|\alpha|}, \quad\left|D^{\alpha} \varphi(x)\right| \leq M_{\alpha}\left(r^{\prime \prime}-r^{\prime}\right)^{-|\alpha|}
$$

dove $M_{\alpha}$ ə̀ una costante dipendente solo da $\alpha$.

Sia ora $u$ una funzion $\Theta$ di classe $V\left(Q_{r_{0}}\right)$.

Nel caso $Q_{r_{0}}=\Gamma_{r_{0}}$, con ben note considerazioni si dimostra che sussiste la limitazione $\left({ }^{26}\right)$

$$
\underset{\langle\alpha, q>\leq m}{\sum}\left|D^{\alpha}(\zeta u)\right|_{2, \Gamma_{r_{0}}} \leq c_{1}\left(|A(\zeta u)|_{2, \Gamma_{r_{0}}}+|\zeta u|_{2, \Gamma_{r_{0}}}\right) .
$$

Nel caso $Q_{r_{0}}=\Sigma_{r_{0}}$, associamo ad $u$ le funzioni

$$
\begin{array}{r}
v(x)\left\{\begin{array}{lll}
=\varphi(x) u(x) & \text { se } & x \in \Sigma_{r_{0}} \\
=0 & \text { se } & x \in R_{+}^{n}-\Sigma_{r_{0}},
\end{array}\right. \\
v_{1}(x)\left\{\begin{array}{lll}
=v(x) & \text { se } \quad x_{n}>0 \\
=\sum_{j=1}^{m_{n}} \lambda_{j} v\left(x^{\prime},-j x_{n}\right) & \text { se } \quad x_{n}<0,
\end{array}\right.
\end{array}
$$

dove $i \lambda_{j}$ sono dei numeri reali tali che

$$
\sum_{j=1}^{m_{n}} \lambda_{j}(-j)^{k}=1, \quad k=0, \ldots, m_{n}-1
$$

Evidentemente si ha che $v \in H^{m}\left(R_{+}^{n}\right)$ e $v_{1} \in H^{m}\left(R^{n}\right)$, dove gli $H^{m}$ sono gli spazi definiti al n. 1.

(26) Nel corso di questa dimostrazione indichiamo con $c_{1}, \ldots, c_{14}$ delle costanti positive indipendenti da $r, r^{\prime}, r^{\prime \prime}$ e $u$. 
Allora dai risultati di T. Matsuzawa [21] si deduce, ancora con note considerazioni, che sussiste la limitazione

$$
\begin{aligned}
& \sum_{<\alpha, q>\leq_{m}}\left|D^{\alpha}(\zeta u)\right|_{2, \Sigma_{r_{0}}} \leq c_{2}|A(\zeta u)|_{2, \Sigma_{r_{0}}} \\
& \left.+\sum_{j=1}^{v}\left\|B_{j}\left(\zeta v_{1}\right)\right\|_{H}^{m-p_{j}-q_{n / 2}\left(R^{n-1}\right)}+|\zeta u|_{2, \Sigma_{r_{0}}}\right) .
\end{aligned}
$$

Osserviamo ora che si ha

$$
\begin{aligned}
& |A(\zeta u)|_{2, Q_{r_{0}}} \leq|\zeta A u|_{2, Q_{r_{0}}}+c_{3} \underset{<\mu, q>\leq m}{\Sigma} \underset{\alpha<\mu}{\Sigma}\left|D^{\mu-\alpha \zeta} \cdot D^{\alpha} u\right|_{2, Q_{r_{0}}} \\
& \quad \leq|\zeta A u|_{2, Q_{r_{0}}}+c_{4}\left(r^{\prime}-r\right)^{-m}{\underset{<\alpha, q><m}{\Sigma}\left|D^{\alpha}(\varphi u)\right|_{2, Q_{r_{0}}}}^{\Sigma}
\end{aligned}
$$

D'altra parte, in conseguenza del lemma 7.1, si ha

$$
\begin{aligned}
& \sum_{<\alpha, q><m}\left|D^{\alpha}(\varphi u)\right|_{2, Q_{r_{0}}} \leq c_{5}\left[\left(\sum_{<\alpha, q>=m}^{\Sigma}\left|D^{\alpha}(\varphi u)\right|_{2, Q_{r_{0}}}\right)^{\alpha_{0}} \cdot|\varphi u|_{2, Q_{r_{0}}}^{1-a_{0}}+|\varphi u|_{2, Q_{r_{0}}}\right] \\
& \leq c_{6}\left[\left(r^{\prime \prime}-r^{\prime}\right)^{-m}\left(\underset{<\alpha, q>\leq m}{\Sigma}\left|D^{\alpha} u\right|_{\left.2, Q_{r^{\prime \prime}}\right)^{a_{0}}}^{\Sigma} \cdot|u|_{2, Q_{r^{\prime \prime}}}^{1-a_{0}}+|u|_{2, Q_{r^{\prime \prime}}}\right]\right.
\end{aligned}
$$

dove

$$
a_{0}=\sup _{\langle\alpha, q>\leq m} \frac{\langle\alpha, q\rangle}{m} .
$$

Dalle $(9.8)$ e (9.9) segue che risulta

$$
\begin{aligned}
& |A(\zeta u)|_{2 . Q_{r_{0}}} \leq c_{7}\left(|A u|_{2, \ell_{r^{\prime \prime}}}+\left(r^{\prime \prime}-r\right)^{-m}|u|_{2, Q_{r^{\prime \prime}}}\right. \\
& +\left(r^{\prime \prime}-r\right)^{-2 m}\left(\underset{<\alpha, q>\leq m}{\Sigma}\left|D^{\alpha} u\right|_{\left.2, Q_{r^{\prime \prime}}\right)^{a_{0}}}|u|_{\left.2, Q_{r^{\prime \prime}}\right)}^{1-a_{0}}\right) .
\end{aligned}
$$

Inoltre, in conseguenza delle (9.2) e dei lemmi 1.7, 1.3, si ha

$$
\begin{aligned}
& \left\|B_{j}\left(\zeta v_{1}\right)\right\|_{H^{m-p_{j}}-q_{n / 2}\left(R^{n-1}\right)} \leq c_{8} \sum_{<\mu, q>\leq p_{j}} \sum_{\alpha<\mu}\left\|D^{\mu-\alpha} \zeta \cdot D^{\alpha} v_{1}\right\|_{A^{m-p_{j}}}{ }_{\left(R^{n}\right)} \\
& \leq c_{9}\left(r^{\prime}-r\right)^{-p_{j}} \underset{<\alpha_{2} q><p_{j}}{\Sigma}\left\|D^{\alpha} v_{1}\right\|_{I I}^{m-p_{j}\left(R^{n}\right)}
\end{aligned}
$$


Con ragionamenti analoghi a quelli tenuti da E. Grostr per stabilire il lemma 4.II della parte I di [16], si dimostra $\operatorname{ch} \theta$, se $\langle\alpha, q\rangle\left\langle p_{j}\right.$, si ha per ogni $\eta>0$

$$
\begin{gathered}
\left\|D^{\alpha} v_{1}\right\|_{H}^{m-p_{j}\left(R^{n}\right)} \leq c_{10}\left(\eta^{-p_{j}}+<\alpha, q>\underset{<\mu, q>=m}{\Sigma}\left|D^{\mu} v_{1}\right|_{2, R^{n}}\right. \\
+\left(1+\eta^{m-p_{j}+\langle\alpha, q>)}\left|v_{1}\right|_{2, R^{n}}\right),
\end{gathered}
$$

da cui, posto

$$
\eta=\left(\underset{<\mu, q>=m}{\Sigma}\left|D^{\mu} v_{1}\right|_{2, R^{n}}\right)^{1 / m} \cdot\left|v_{1}\right|_{2, R^{n}}^{-1 / m}
$$

si ricava

$$
\begin{aligned}
& \left\|D^{\alpha} v_{1}\right\|_{H}{ }^{\left.m-p_{j} R^{n}\right)} \leq c_{11}\left(\left|v_{1}\right|_{2, R^{n}}\right.
\end{aligned}
$$

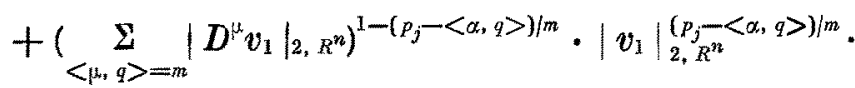

Se poniamo

$$
b_{j}=\sup _{<\alpha, q><p_{j}}\left(1-\frac{p_{j}-<\alpha, q>}{m}\right), \quad b_{0}=\sup \left(b_{1}, \ldots, b_{\nu}\right\}
$$

dalle (9.11) e (9.12) si deduce che riesce

$$
\begin{aligned}
& \sum_{j=1}^{\nu}\left\|B_{j}\left(\zeta v_{1}\right)\right\|_{H^{m-P_{j}}-q_{n / 2}\left(R^{n-1}\right)} \leq c_{12}\left(r^{\prime}-r\right)^{-m}\left(|\varphi u|_{2, Q_{r_{0}}}\right. \\
& \left.+\left(\underset{<\alpha, q>=m}{\Sigma}\left|D^{x}(\varphi u)\right|_{2,}, Q_{r_{0}}\right)^{b_{0}} \cdot|\varphi u|_{2, Q_{r_{0}}}^{1-b_{0}}\right) \\
& \leq c_{13}\left(r^{\prime}-r\right)^{-2 m}\left(|u|_{2, Q_{r^{\prime \prime}}}+\left(\sum_{<\alpha, q>\leq m}^{\Sigma}\left|D^{\alpha} u\right|_{2, Q_{r^{\prime \prime}}}\right)^{b_{0}} \cdot|u|_{2, Q_{r^{\prime \prime}}}^{1-b_{0}}\right) .
\end{aligned}
$$

Dalle (9.6), (9.7), (9.10), (9.13) e dal lemma 7.1 segue che, denotato con $\gamma$ il più grande dei due numeri $a_{0}$ e $b_{0}$, si ha

$$
\begin{aligned}
& \left(r^{\prime \prime}-r\right)^{2 m} \underset{<\alpha, q>=m}{\Sigma}\left|D^{\alpha} u\right|_{2, Q_{r}} \leq c_{14}\left(|A u|_{2, Q_{r^{\prime \prime}}}+|u|_{2, Q_{r^{\prime \prime}}}\right. \\
& +\left(\left.\underset{<\alpha, q>=m}{\Sigma}\left|D^{\alpha} u\left(2, Q_{r^{\prime \prime}}\right)^{\gamma} \cdot\right| u\right|_{\left.2, Q_{r^{\prime \prime}}\right)} ^{1-\Psi^{\gamma}}\right.
\end{aligned}
$$


Da quest' ultima relazione e da un lemma di crescenza di C. Miranda (cfr. il lemma 3.1 di [23]) si deduce la tesi.

Dimostrazione del teorema 8.1. - Fissato comunque $x_{0} \in \Omega$ ed assegnata una funzione $u(x)$ soddisfacente le ipotesi poste, consideriamo la funzione $u^{*}\left(x_{0}, \xi\right)$ definita in $I^{*}\left(x_{0}\right)$ dalla (7.7).

Cominciamo col dimostrare che esiste una costante $c_{0}$, indipendente da $u$ e $x_{0}$, tale che si ha

$$
\underset{<\alpha, q>=m}{\Sigma}\left|D^{\alpha} u^{*}\right|_{2, I_{1 / 2}^{*}\left(x_{0}\right)} \leq c_{0}\left(\left|\tilde{A} u^{*}\right|_{2, I^{*}\left(x_{0}\right)}+\left|u^{*}\right|_{2, I^{*}\left(x_{0}\right)}\right)
$$

dove $\tilde{A}$ ̀̀ l'operatore definito dalla $(8.8)$ e

$$
I_{1 / 2}\left(x_{0}\right)=\Omega \cap\left\{x \in R^{n}|| x^{(i)}-x_{0}^{(i)} \mid<\frac{1}{2} \rho^{\tau^{(i)}}\left(x_{0}\right), i=1, \ldots, t\right\} .
$$

Dall'ipotesi $\mathcal{H}_{1}$ ) e dalle (7.2), (7.3) si deduce ohe $\tilde{A}$ è quasi-ellittico in $\overline{I^{*}\left(x_{0}\right)}$ e che i suoi coefficienti

$$
\rho^{x-<\mu, \tau>}\left(x_{0}\right) a_{\mu}^{*}\left(x_{0}, \xi\right)=\left(\frac{\rho^{*}\left(x_{0}, \xi\right)}{\rho\left(x_{0}\right)}\right)^{<\mu, \tau>-\chi} \rho^{* \chi \chi-<\mu . \tau>}\left(x_{0}, \xi\right) a^{*}\left(x_{0}, \xi\right)
$$

per $\langle\mu, q\rangle=m$ sono continui rispetto a $\xi$ in $\overline{I^{*}\left(x_{0}\right)}$ uniformemente al variare di $x_{0}$ in $\Omega\left({ }^{17}\right)$ e per $\langle\mu, q\rangle\left\langle m\right.$ hanno moduli limitati rispetto a $\xi$ in $I^{*}\left(x_{0}\right)$ da una costante indipendente da $x_{0}$.

Pertanto, se $\Gamma\left(x_{0}\right)=\varnothing$, la $(9.15)$ si ottiene come conseguenza del lemma 9.1 applicato relativamente al caso in cui $Q_{r_{0}}=\Gamma_{r_{0}}=I^{*}\left(x_{0}\right)$.

Consideriamo allora il caso che sia $\Gamma\left(x_{0}\right) \neq \emptyset$. In tal caso la funzione $u^{*}\left(x_{0}, \xi\right)$ soddisfa le condizioni

$$
\tilde{B}_{i j} u^{*}=0 \quad \text { su } \quad \Gamma_{i}^{*}\left(x_{0}\right), \quad j=1 . \ldots, \gamma_{i},
$$

per quei valori di $i$ per cui $\Gamma_{i}\left(x_{0}\right) \neq \varnothing$, dove gli operatori $\tilde{B}_{i j}$ sono definiti dalla (8.9).

(17) Si tenga presente l'osservazione 8.1. 
Procedendo come nella dimostrazione del teorema 2.1 di [34], consideriamo un ricoprimento di $I_{1 / 2}^{*}\left(x_{0}\right)$ mediante un numero finito $\mathcal{C}_{1}, \ldots, \mathfrak{C}_{r}$ di aperti di $R^{n}$ in modo che ogni $\mathcal{C}_{k}$ soddisfi una delle seguenti condizioni:

1) $\overline{\mathfrak{C}}_{k} \subset I^{*}\left(x_{0}\right)$;

1') $\mathfrak{C}_{k}$ è contenuto in uno degli aperti $\mathcal{O}_{i \hbar}$ definiti nel corso dell'osservazione 7.2 .

Se $\overline{\mathfrak{C}}_{k} \subset I^{*}\left(x_{0}\right)$, ancora come conseguenza del lemma 9.1 applicato relativamente al caso in oui $Q_{r_{0}}=\Gamma_{r_{0}}$, si ottiene la limitazione

$$
\underset{<\alpha, q>=m}{\Sigma}\left|D^{\alpha} u^{*}\right|_{2, \mathfrak{C}_{k}} \leq c^{\prime}\left(\left|\tilde{A} u^{*}\right|_{2, I^{*}\left(x_{0}\right)}+\left|u^{*}\right|_{2, I^{*}\left(x_{0}\right)}\right),
$$

dove $c^{\prime}$ è una costante indipendente da $u$ e da $x_{0}$.

Nel caso $1^{\prime}$ ) si ottiene ancora la (9.17) trasformando l'insieme $\mathcal{O}_{i h} \cap I^{*}\left(x_{0}\right)$ sull'insieme $\Sigma^{(i)}$ mediante l'omeomorfismo $\varphi_{i h}$, tenendo presente le proprietà di $\varphi_{i h}$, tenendo conto dell'osservazione 8.2 e facendo uso del lemma 9.1 relativamente al caso in cui $Q_{r_{0}}=\Sigma_{r_{0}}$.

La (9.17) valida per $k=1, \ldots, r$ implica la (9.15).

Operando ora in $(9.15)$ il cambiamento di variabili (7.6) si ha

$$
\begin{gathered}
\sum_{<\alpha, q>=m} \rho^{<\alpha, \tau>-|\tau| / 2}\left(x_{0}\right)\left|D^{\alpha} u\right|_{2, I_{1 / 2}\left(x_{0}\right)} \leq c_{0}\left(\rho^{\gamma-|\tau| / 2}\left(x_{0}\right)|A u|_{2, I\left(x_{0}\right)}\right. \\
+\rho^{-|\tau| / 2}\left(x_{0}\right)|u|_{\left.2, I\left(x_{0}\right)\right)} .
\end{gathered}
$$

Moltiplicando primo e secondo membro della (9.18) per $\rho^{s}\left(x_{0}\right)$, elevando al quadrato ed integrando su $\Omega$ si ha

$$
\begin{aligned}
& \sum_{\langle\alpha, q>=m} \int_{\Omega}\left(\rho^{s+<\alpha, \tau\rangle-|\tau| / 2}\left(x_{0}\right)\left|D^{\alpha} u\right|_{2, I_{1 / 2}\left(x_{0}\right)}\right)^{2} d x_{0} \\
& \leq c_{1}\left(\int_{\Omega}\left(\rho^{s+x-|\tau| / 2}\left(x_{0}\right)|A u|_{2, I\left(x_{0}\right)}\right)^{2} d x_{0}+\int_{\Omega}\left(\rho^{s-\left.|\tau|\right|^{2}}\left(x_{0}\right)|u|_{2, I\left(x_{0}\right)}\right)^{2} d x_{0}\right),
\end{aligned}
$$

dove $c_{1}$ è una costante indipendente da $u$.

Dalla $(9.19)$ e dai lemmi $7.3,7.4$ si deduce la limitazione

$$
\underset{<\alpha, q>=m}{\Sigma}\left|D^{\alpha} u\right|_{2, s+<\alpha, \tau>} \leq c_{2}\left(|A u|_{2, s+\chi}+|u|_{2, s}\right),
$$

dove $c_{2}$ è una costante indipendente da $u$.

Dalla (9.20) e dal lemma 7.5 segue la (8.14) e quindi si ha la tesi. 


\section{0. - Dimostrazione del teorema 8.2.}

Premettiamo il seguente lemma, che costituisce una generalizzazione del lemma 3.1 di [33] e dove facciamo uso delle notazioni iniziali del n. 9:

Leman 10.1. - Supponiamo che:

$\left.i_{1}\right)$ siano verificate le ipotesi del lemma 9.1 ;

$\left.i_{2}\right)$ esistano $\theta$ e $l$ tali che l'operatore $\mathfrak{L}_{\theta, l}\left(x, D_{x}, D_{y}\right)$, associato ad $A\left(x, D_{x}\right)$ tramite la $(8.18)$, sia quasi-ellittico in $\bar{Q}_{r_{0}} \times R^{1}$ e gli operatori $B_{1}\left(x, D_{x}\right)$, $\ldots, B_{y}\left(x, D_{x}\right)$ verifichino la condizione complementare rispetto a $\varrho_{\theta,}\left(x, D_{x}, D_{y}\right)$ su $\bar{\sigma}_{r_{0}} \times R^{1}$.

Allora per ogni $u \in V\left(Q_{r_{0}}\right)$ e per ogni numero reale $\eta$ sussiste la limitazione

$$
\begin{aligned}
& \sum_{k=0}^{l}|\eta|_{<\alpha, q>\leq m(l-k / l)}^{k}\left|D^{\alpha} u\right|_{2, Q_{r}} \leq c\left(\left|\left(A+\eta^{l} e^{i \theta}\right) u\right|_{2, Q_{\tau_{0}}}\right. \\
& \left.+\left(1+|\eta|^{l-1}\right)|u|_{2,}, Q_{r_{0}}\right)
\end{aligned}
$$

dove $r<r_{0}$ e dove c è una costante indipendente da $u$ e da $\eta$.

Poggiando sul lemma 9.1, otterremo la dimostrazione di questo lemma adattando al nostro caso un noto procedimento dovuto a S. AGMON [1].

Siano $u(x)$ una funzione di classe $V\left(Q_{r_{0}}\right)$ e $\eta$ un numero reale.

Assegnata $\zeta(y) \in C_{0}^{\infty}\left(R^{1}\right)$ e tale che

$$
\zeta(y)\left\{\begin{array}{lll}
=1 & \text { per } & |y| \leq 1 / 2 \\
=0 & \text { per } & |y| \geq 1
\end{array}\right.
$$

poniamo

$$
v_{\eta}(x, y)=\zeta(y) e^{i \eta y} u(x)
$$

Evidentemente, in conseguenza del lemma 9.1 si ha la limitazione

$$
\left.\underset{\langle\alpha, q\rangle+m k i l \leq m}{\sum} \mid D_{x}^{\alpha} D_{y}^{k} v_{\eta}\right]_{2, Q_{r} \times R^{1}} \leq c_{1}\left(\left|\mathcal{L}_{\theta, i} v_{\eta}\right|_{2, Q_{r_{0}} \times R^{1}}+\left|v_{\eta}\right|_{2, Q_{r_{0}} \times R^{1}}\right)
$$

dove $r$ è un fissato numero reale positivo $<r_{0}$ e $c_{1}$ è una costante indipendente da $u$ e da $\eta$. 
Osserviamo che si ha

$$
\mathfrak{L}_{\theta, l} v_{n}=\zeta(y) e^{i \eta y}\left(A+\eta^{i} e^{i \theta}\right) u+e^{i \theta} e^{i \eta y} u \cdot \sum_{k=0}^{l-1}\left(\begin{array}{l}
l \\
k
\end{array}\right) D_{y}^{l-k} \zeta(y) \eta^{k}
$$

e quindi riesce

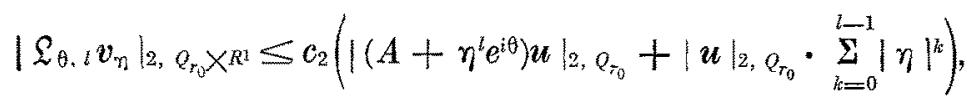

dove $c_{2}$ è una costante indipendente da $u$ e da $\eta$.

Inoltre si ha

da cui segue

$$
\begin{aligned}
\left|D_{x}^{\alpha} D_{y}^{k} v_{\eta}\right|_{2, Q_{r} \times R^{2}}^{2} & \geq \int_{Q_{r}} d x \int_{-1 / 2}^{1 / 2}\left|D_{x}^{\alpha} D_{y}^{k}\left(e^{i \eta y} u(x)\right)\right|^{2} d y \\
& =|\eta|^{2 k} \int_{Q_{r}}\left|D_{x}^{\alpha} u\right|^{2} d x,
\end{aligned}
$$

$$
\sum_{<\alpha, q>+m k \mid l \leq m}\left|D_{x}^{\alpha} D_{y}^{k} v_{\eta}\right| 2, Q_{r} \times R^{1} \geq \sum_{k=0}^{l}|\eta|^{k} \underset{<\alpha, q>\leq m(1-k / l)}{\Sigma}\left|D_{x}^{\alpha} u\right|_{2, Q_{r}} .
$$

Dalle (10.2), (10.3) e (10.4) si deduce la (10.1) e quindi si ha la tesi.

Dimostrazione del teorema 8.2. - Consideriamo l'operatore

$$
\widehat{A}=\left(\frac{\sigma^{*}\left(x_{0}, \xi\right)}{\rho\left(x_{0}\right)}\right)^{x} \tilde{A}
$$

dove $\tilde{A}$ ̀े definito dalla (8.8), ed assegnamo una funzione $u$ di classe $W_{s}^{m}(\Omega)$ e soddisfacente le (8.17).

Tenendo presente l'osservazione 8.1 , con considerazioni analoghe a quelle tenute nel corso della dimostrazione del teorema 8.1 per dedurre dal lemma 9.1 la $(9.15)$, si deduce ora dal lemma 10.1 che per ogni $x_{0} \in \Omega$ e per ogni numero reale $\eta$ sussiste la limitazione

$$
\begin{gathered}
\sum_{k=0}^{l}|\eta|_{<\alpha, q>\leq m(1-\bar{k} /)}^{k}\left|D^{x} u^{*}\right|_{2, I_{1 / 2}^{*}\left(x_{0}\right)} \\
\leq c_{1}\left(\left|\left(\bar{A}+\eta^{l} e^{i \theta}\right) u^{*}\right|_{2, I^{*}\left(x_{0}\right)}+\left(1+|\eta|^{l-1}\right)\left|u^{*}\right|_{2, I^{*}\left(x_{0}\right)}\right),
\end{gathered}
$$

dove $c_{1}$ è una costante indipendente da $x_{0}, u$ e $\eta$. 
Inoltre, con considerazioni analoghe a quelle tenute per dedurre dalla (9.15) la (9.19), si deduce dalla (10.5) che si ha

$$
\begin{aligned}
& \sum_{k=0}^{l}|\eta|^{2 k} \underset{<\alpha, q>\leq \leq m(1-k / l)}{\mathbf{S}} \int_{\mathbf{\Omega}}\left(\rho^{s-m+<\alpha, \tau>-|\tau| / 2}\left(x_{0}\right)\left|D^{\alpha} u\right|_{\mid 2, I_{1 / 2}\left(x_{0}\right)}\right)^{2} d x_{0} \\
& \leq c_{2}\left[\int_{\Omega}\left(\rho^{s-m-i \tau \mid / 2}\left(x_{0}\right)\left|\left(\sigma^{\chi} A+\eta^{l} e^{i \theta}\right) u\right|_{2, I\left(x_{0}\right)}\right)^{2} d x_{0}\right. \\
& \left.+\left(1+|\eta|^{\mid-1}\right)^{2} \int_{\Omega}\left(\rho^{s-m-|\tau| / 2}\left(x_{0}\right)|u|_{2, I\left(x_{0}\right)}\right)^{2} d x_{0}\right],
\end{aligned}
$$

dove $c_{2}$ è una costante indipendente da $u, \eta$ e $s$.

Dalle (10.6), (8.10), (7.3) e dai lemmi 7.3, 7.4 segue la limitazione

$$
\begin{gathered}
\sum_{k=0}^{l}|\eta|_{<\alpha, q>S_{m(1-k / \ell)}^{k}}^{\Sigma}\left|D^{\alpha} u\right|_{2, s+<x, \tau>-m} \\
\leq c_{3} d_{0}^{2 \mid s}\left(\left|\left(A+\eta^{l} e^{i \theta} \sigma-X\right) u\right|_{2, s+\chi-m}+\left(1+|\eta|^{l-1}\right)|u|_{2, s-m}\right),
\end{gathered}
$$

dove $c_{3}$ è una costante indipendente da $u$, $\eta$ e $s$.

Dalla (10.7) si deduce in modo ovvio la tesi assumendo $\lambda=\eta^{i} e^{i \phi}$.

\section{1. - La formula di Green; dimostrazione del teorema 8.3.}

Diremo che un operatore di frontiera $B_{i j}$ della forma $(8.5)$ e definito su $\partial \Omega$ è di classe $\mathfrak{J B}^{\left(\gamma_{i}, \tau\right)}$, se i suoi coefficienti verificano l'ipotesi $\mathfrak{H}_{3}$ ); chiameremo $q$-ordine di $B_{i j}$ il numero $p_{i j}$.

Inoltre diremo che un sistema $\left\{B_{j}\right\}_{j=1}^{r}$ di $r$ operatori di frontiera della forma (8.20) ̀̀ un sistema di Dirichlet di ordine $r$ su una porzione $\Gamma$ di $\overline{\partial_{i} \Omega}$, se esso è normale su $\Gamma$ e i numeri $p_{j}$ sono $\leq(r-1) q^{(i)}$.

Indicheremo con $A^{*}$ l'operatore aggiunto formale di $A$ :

$$
A^{*} u=\underset{<\mu, q>\leq m}{\Sigma} D^{\mu}\left(\bar{a}_{\mu}(x) u\right)
$$

Leman 11.1. - Siano verificate le ipotesi $\left.\left.\left.\left.a_{0}\right), \mathscr{H}_{1}\right), \mathscr{H}_{3}\right), \mathscr{H}_{6}\right), \mathfrak{H}_{7}$ ) e siano assegnati gli operatori $C_{i j}, j=1, \ldots, m^{(i)}-v_{i}$ e $i \in \mathfrak{I}$, di q-ordine $n_{i j}$, di classe 
$\mathfrak{H B}^{\left(Y_{i}, \tau\right)}$ e tali che, per ogni $i \in \mathfrak{J}$, gli operatori $B_{i j}^{\left(Y_{i j}, \tau\right)}$ e $C_{i j}^{\left(Y_{i j}, \tau\right)}$ costituiscano un sislenıa di Dirichlet di ordine $m^{(i)} s u \overline{\partial_{i} \Omega}$.

Allora, per ogni $i \in \mathfrak{J}$, esistono due sistemi $\left\{B_{i j}^{\prime}\right\}_{j=1}^{m^{(i)}-v_{i}}$ e $\left\{O_{i j}^{\prime}\right\}_{j=1}^{\nu_{i}}$ di operator $i$ differenziali lineari di frontiera verificanti le seguenti condizioni:

1) $B_{i j}^{\prime}$ e $C_{i j}^{\prime}$ sono operatori di classe $\mathfrak{B}^{\left(x-r_{i}-m^{(i)_{\tau}} \tau^{(i)}, \tau\right)}$, rispeltivamente di $q$-ordine $m^{(i)}-n_{i j}-1$ e $m^{(i)}-p_{i j}-1$;

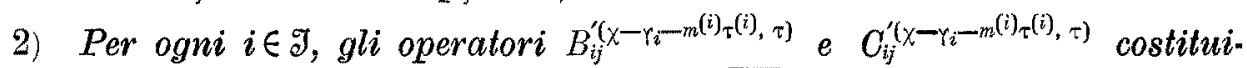
scono un sistema di Dirichlet di ordine $m^{(i)}$ su $\overline{\partial_{i} \Omega \text {; }}$

3) Per ogni coppia di funzioni $u$ e $v$ di classe $C_{0}^{\infty}(\bar{\Omega}-S)$ sussiste la formula di Green:

$$
\int_{\Omega} A u \cdot \vec{v} d x-\int_{\Omega} u \cdot \overline{A^{*} v} d x=\sum_{i \in \mathfrak{g}}\left(\sum_{j=1}^{m^{(i)}} \int_{\partial_{i} \Omega} C_{i j} u \cdot \overline{B_{i j}^{\prime} v} d \sigma-\sum_{j=1}^{y_{i}} \int_{\partial_{i} \Omega} B_{i j} u \cdot \overline{C_{i j}^{\prime} v} d \sigma\right)
$$

4) Se, fissato $i \in \mathfrak{J}$, A è propriamente quasi-ellittico di tipo $\gamma_{i}$ su $\partial_{i} \Omega$, allora gli operatori $B_{i 1}^{\prime}, \ldots, B_{i, m_{(i)}{ }_{-v_{i}}}$ verificano la condizione complementare rispetto ad $A^{*}$ su $\partial_{i} \Omega$ se e solo se gli operatori $B_{i 1}, \ldots, B_{i y_{i}}$ verificano la stessa condizione rispetto ad $A$ su $\partial_{i} \Omega$.

Infatti, siano $\mathcal{Q}_{i k}$ gli insiemi definiti nell' osservazione 7.1.

Dai risultati del n. 6 , si deduce evidentemente che ad ogni $\mathscr{U}_{i k}$ è possibile associare due sistemi $\left\{B_{i k_{j}}^{\prime}\right\}_{j=1}^{m^{(i)}-y_{i}}$ e $\left\{C_{i k j}^{\prime}\right\}_{j=1}^{\nu_{i}}$ di operatori soddisfacenti, limitatamente alla porzione $\partial_{i} \Omega \cap \mathcal{L}_{i k}$ di $\partial_{i} \Omega$, alle condizioni 1), 2) e 4) del teorema e tali che per $f, g \in C^{\infty}(\bar{\Omega})$, con $f$ oppure $g$ a supporto compatto in $\bar{\Omega} \cap 2 \mathcal{R}_{i k}$, si abbia

$$
\begin{gathered}
\int_{\Omega} A f \cdot \bar{g} d x-\int_{\Omega} f \cdot \overline{A^{*} g} d x \\
=\sum_{j=1}^{m(i)_{-y_{i}}} \int_{\delta_{i^{\Omega}}} C_{i j} f \cdot \overline{B_{i k j}^{\prime} g} d \sigma-\sum_{j=1}^{\nu_{i}} \int_{\partial_{i} \Omega} B_{i j} f \cdot \overline{C_{i k j}^{\prime} g} d \sigma .
\end{gathered}
$$

Assegnamo un ricoprimento di $S$ mediante un numero finito $\mathfrak{C}_{1}, \ldots, \mathfrak{C}_{p}$ di aperti di $R^{n}$ tali che, per ogni $r \in\{1, \ldots, p\}$ e per ogni $i \in \mathfrak{g}$, l'insieme 
$\mathcal{C}_{r} \cap \partial_{i} \Omega$, se non vaoto, sia contenuto in uno degli insiemi $\mathscr{Q}_{i k}$.

Assegnamo inoltre un numero finito $\mathcal{O}_{I}, \ldots, \Theta_{s}$ di aperti di $R^{n}$ tali che $\overline{\mathcal{O}}_{k} \subset \Omega$ e con la condizione che gli insiemi $\mathcal{O}_{k}, \mathscr{Q}_{i k}$ e $\mathcal{C}_{k}$ costituiscano un ricoprimento di $\bar{\Omega}$.

Consideriamo quindi una partizione dell'nnità su $\bar{\Omega}$ relativa al suddetto ricoprimento e costituita di funzioni $\alpha_{k}, \beta_{i k}, \varphi_{k}$ di classe $C_{0}^{\infty}\left(R^{n}\right)$ e tali che

$$
\operatorname{supp} \alpha_{k} \subset \mathcal{O}_{k}, \quad \operatorname{supp} \beta_{i k} \subset \mathcal{Q}_{i k}, \quad \operatorname{supp} \varphi_{k} \subset \mathcal{C}_{k} .
$$

Fissate allora $u, v \in O_{0}^{\infty}(\bar{\Omega}-S)$, si ha

$$
\begin{gathered}
\int_{\Omega} A u \cdot \bar{v} d x=\sum_{k=1}^{s} \int_{\Omega} A u \cdot \overline{\alpha_{k} v} d x \\
+\sum_{i \in \mathfrak{g} j} \sum_{k=1}^{h_{i}} \int_{\Omega} A u \cdot \overline{\beta_{i k} v} d x+\sum_{k=1}^{p} \int_{\Omega} A u \cdot \overline{\varphi_{k} v} d x .
\end{gathered}
$$

Evidentemente risulta

$$
\int_{\Omega} A u \cdot \overline{\alpha_{k}} v d x=\int_{\Omega^{-}} u \cdot \overline{A^{*}\left(\alpha_{k} v\right)} d x,
$$

mentre in conseguenza della (11.3) si ha

$$
\begin{gathered}
\int_{\Omega} A u \cdot \overline{\beta_{i k} v} d x-\int_{\Omega} u \cdot \overline{A^{*}\left(\beta_{i k} v\right)} d x \\
=\sum_{j=1}^{m^{(i)}-y_{i}} \int_{\partial_{i} \Omega} C_{i j} u \cdot \overline{B_{i k j}^{\prime}\left(\beta_{i k} v\right)} d \sigma-\sum_{j=1}^{\nu_{i}} \int_{\partial_{i} \Omega} B_{i j} u \cdot \overline{O_{i k j}^{\prime}\left(\beta_{i k} v\right)} d \sigma .
\end{gathered}
$$

Volendo trasformare l'ultimo integrale a secondo membro della (11.4), indichiamo con $T$ il supporto di $v$ e con $E_{k}$ il supposto di $\varphi_{k}$.

Assegnamo un ricoprimento di $T \cap E_{k}$ con un numero finito $\mathfrak{D}_{k 1}, \ldots, \mathfrak{D}_{k_{k}}$ di aperti di $R^{n}$ in modo che ogni $\mathfrak{D}_{k r}$ verifichi una delle seguenti due condizioni :

a) $\overline{\mathfrak{D}_{k r}} \subset \Omega$; 
$\left.a^{\prime}\right)$ esiste $\mathcal{Q}_{i h}$ tale che $\overline{\mathfrak{D}_{k r}} \cap\left(T \cap E_{k}\right) \subseteq \overline{\mathcal{Q R}_{i h}} \cap T$ e $\mathfrak{D}_{k r} \supset \overline{\mathcal{Q}_{i h}} \cap \overline{\partial_{i} \Omega}$.

Consideriamo quindi una partizione dell'unità su $T \cap E_{k}$ relativa al suddetto ricoprimento e costituita di funzioni $\zeta_{k r} \in C_{0}^{\infty}\left(R^{n}\right)$, a supporto compatto in $\mathfrak{D}_{k r}$ e tali che nel caso $a^{\prime}$ ) riesca

$$
\zeta_{k r}=1 \quad \text { su } \quad E_{k} \cap \overline{\partial_{i} \Omega}
$$

Allora si può scrivere

$$
\int_{\Omega} A u \cdot \overline{\varphi_{k} v} d x=\sum_{r=1}^{r_{k}} \int_{\Omega} A u \cdot \overline{\zeta_{k r} \varphi_{k} v} d x .
$$

Nel caso a) si ha evidentemente

$$
\int_{\mathbf{\Omega}} A u \cdot \overline{\zeta_{k r} \varphi_{k} v} d x=\int_{\mathbf{\Omega}} u \cdot \overline{A^{*}\left(\zeta_{k r} \varphi_{k} v\right)} d x .
$$

Nel caso $a^{\prime}$ ) si può evidentemente applicare la (11.3), e quindi si ha

$$
\begin{gathered}
\int_{\Omega} A u \cdot \overline{\zeta_{k r} \varphi_{k} v} d x-\int_{\Omega} u \cdot \overline{A^{*}\left(\zeta_{k r} \varphi_{k} v\right)} d x \\
=\sum_{j=1}^{m^{(i)}-v_{i}} \int_{\partial_{i} \Omega} C_{i j} u \cdot \overline{B_{i k j}^{\prime}\left(\zeta_{k r} \varphi_{h} v\right)} d \sigma-\sum_{j=1}^{v_{i}} \int_{j_{i} \Omega} B_{i j} u \cdot \overline{C_{i k j}^{\prime}\left(\zeta_{k r} \varphi_{k} v\right)} d \sigma .
\end{gathered}
$$

Ne segue che risulta

$$
\begin{gathered}
\int_{\Omega} A u \cdot \overline{\varphi_{k} v} d x-\int_{\Omega} u \cdot \overline{A^{*}\left(\varphi_{k} v\right)} d x \\
=\sum_{i \in \mathfrak{I}}\left(\sum_{j=1}^{m^{(i)}{ }_{j}^{-y_{i}}} \int_{\boldsymbol{d}_{i} \Omega} C_{i j} u \cdot \overline{B_{i k}^{\prime}\left(\varphi_{k} v\right)} d \sigma-\sum_{j=1}^{y_{i}} \int_{\partial_{i} \Omega} B_{i j} u \cdot \overline{C_{i k_{j}}^{\prime}\left(\varphi_{k} v\right)} d \sigma\right) .
\end{gathered}
$$

Dalle (11.4)-(11.7) e dalle proprietà degli operatori $B_{i k j}^{\prime}$ e $C_{i k j}^{\prime}$ si deduce che gli operatori $B_{i j}^{\prime}$ e $C_{i j}^{\prime}$ definiti dalle formule 


$$
\begin{aligned}
& B_{i j}^{\prime} v=\sum_{k=1}^{h_{i}} B_{i k j}^{\prime}\left(\beta_{i k} v\right)+\sum_{k=1}^{P} B_{i k j}^{\prime}\left(\varphi_{k} v\right), \\
& C_{i j}^{\prime} v=\sum_{k=1}^{h_{i}} C_{i k j}^{\prime}\left(\beta_{i k} v\right)+\sum_{k=1}^{p} C_{i k j}^{\prime}\left(\varphi_{k} v\right),
\end{aligned}
$$

soddisfano le condizioni richieste dal lemma.

Lema 11.2. - Nelle stesse ipotesi del lemma 11.1 si ha:

a) La formula di Green (11.2) sussiste per ogni coppia di funzioni u e v, una di classe $C_{0}^{\infty}(\bar{\Omega}-S)$ e l'altra di classe $W^{m}(T)$ per ogni compatto $T \subset \overline{\mathbf{\Omega}}-S$.

b) Se $v \in W^{m}(T)$ per ogni compatto $T \subset \bar{\Omega}-S$, si ha $B_{i j}^{\prime} v=0$ su $\partial_{i} \Omega$, $j=1, \ldots, m^{(i)}-v_{i}$, se e solo se risulta

$$
\int_{\mathbf{\Omega}} A u \cdot \bar{v} d x=\int_{\mathbf{\Omega}} u \cdot \overline{A^{*} v} d x
$$

per ogni $u \in C_{0}^{\infty}\left(\Omega \cup \partial_{i} \Omega\right)$ e tale che $B_{i j} u=0$ su $\partial_{i} \Omega, j=1, \ldots, v_{i}$.

c) Se $u \in W^{m}(T)$ per ogni compatto $T \subset \bar{\Omega}-S$, si ha $B_{i j} u=0$ su $\partial_{i} \Omega$, $j=1, \ldots, \gamma_{i}$, se e solo se è verificata la (11.8) per ogni $v \in C_{0}^{\infty}\left(\Omega \cup \partial_{i} \Omega\right)$ e tale che $B_{i j}^{\prime} v=0 s u \partial_{i} \Omega, j=1, \ldots, m^{(i)}-v_{i}$.

Infatti, osserviamo che, in conseguenza del lemma 7.2, ogni funzione $w \in \stackrel{\circ}{W}^{m}(\Omega)$ può essere approssimata in $W^{m}(\Omega)$ da una successione di funzioni di classe $C_{0}^{\infty}(\bar{\Omega}-S)$.

Osserviamo inoltre che, in conseguenza del lemma 6.2, comunque si assegnano le funzioni $f_{j}, j=1, \ldots, v_{i}$, e $g_{j}, j=1, \ldots, m^{(i)}-v_{i}$, di classe $C_{0}^{\infty}\left(\partial_{i} \Omega\right)$, esiste $u \in C_{0}^{\infty}\left(\Omega \cup \partial_{i} \Omega\right)$ tale che

$$
\begin{array}{lll}
B_{i j} u=f_{j} \quad \text { su } & \partial_{i} \Omega, & j=1, \ldots, v_{i}, \\
C_{i j} u=g_{j} & \text { su } \quad \partial_{i} \Omega, & j=1, \ldots, m^{(i)}-v_{i},
\end{array}
$$

ed esiste $v \in C_{0}^{\infty}\left(\Omega \cup \partial_{i} \Omega\right)$ tale che

$$
\begin{aligned}
& B_{i j}^{\prime} v=g_{j} \quad \text { su } \quad \partial_{i} \Omega, \quad j=1, \ldots, m^{(i)}-y_{i} \\
& C_{i j}^{\prime} v=f_{j} \quad \text { su } \quad \partial_{i} \Omega, \quad j=1, \ldots, v_{i} .
\end{aligned}
$$


Dopo tali osservazioni, la dimostrazione del lemma si ottiene con considerazioni analoghe a quelle tenute in [34] per stabilire il corollario 5.1.

Quando sono verificate le condizioni del lemma 11.1, daremo il nome di problema aggiunto formale di $(8.15)-(8.17)$ al problema

$$
\begin{aligned}
& v \in W_{-s-\chi+2 m}^{m}(\Omega), \\
& A^{*} v+\bar{\lambda} \sigma^{-\chi} v=g \quad \text { in } \Omega, \quad g \in L_{-s+m}^{2}(\Omega), \\
& B_{i j}^{\prime} v=0 \quad \text { su } \partial_{i} \Omega, \quad j=1, \ldots, m^{(i)}-\gamma_{i}, \quad i \in \mathfrak{I},
\end{aligned}
$$

e indicheremo con $N_{s, \lambda}^{*}$ l'insieme delle soluzioni del problema omogeneo associato a $(11.9)$.

Inoltre indicheremo:

con $\mathfrak{Q}_{s, \lambda}$ l'operatore $u \rightarrow A u+\lambda \sigma^{-\chi} u$ lineare e continuo da

$$
W_{s}^{m}\left(\Omega, B_{i j}\right)=\left\{u \in W_{s}^{m}(\Omega) \mid B_{i j} u=0 \text { su } \partial_{i} \Omega, j=1, \ldots, v_{i}, i \in \mathfrak{I}\right)
$$

in $L_{s+\chi-m}^{2}(\Omega)$;

con $\mathfrak{Q}_{s, \lambda}^{*}$ l'operatore aggiunto di $\mathfrak{A}_{s, \lambda}$;

oon $\mathscr{T}_{s, \lambda}^{*}$ il nucleo di $\mathfrak{A}_{s, \lambda}^{*}$.

LEMmA 11.3. - Siano verificate le ipotesi $\left.\left.\left.\left.\mathscr{H}_{1}\right)-\mathscr{H}_{4}\right), \mathscr{H}_{6}\right), \mathscr{H}_{7}\right)$ ed inoltre $\sigma(x) \in O^{\infty}(\bar{\Omega}-S)$. Allora per ogni $s$ reale e per ogni $\lambda$ complesso si ha $\mathscr{O Z}_{s, \lambda}^{*}$ $\subseteq N_{s, \lambda}^{*}$.

Se inoltre è anche verificata l'ipotesi $\mathfrak{H}_{5}$ ), si ha $\mathscr{\vartheta}_{s, \lambda}^{*}=N_{s, \lambda}^{*}$.

Infatti, cominciamo con l'osservare che per ogni $s$ reale riesce $L_{-s}^{2}(\Omega)=$ $=\left(L_{s}^{2}(\Omega)\right)^{\prime}\left(=\right.$ duale forte di $\left.L_{s}^{2}(\Omega)\right)\left({ }^{18}\right)$ e che dalla definizione di operatore aggiunto segue che $v \in \mathscr{T}_{s, \lambda}^{*}$ se e solo se si ha

$$
v \in L_{-s-\chi+m}^{2}(\Omega) \text { e } \int_{\Omega} v \cdot \overline{A u+\lambda \sigma^{-\chi} u} d x=0 \quad \forall u \in W_{s}^{m}\left(\Omega, B_{i j}\right) .
$$

D'altra parte dal corollario 5.2, dal lemma 11.2 e dal teorema 8.1 si deduce, con note considerazioni, che una soluzione $v$ di (11.10) è anche una soluzione del problema omogeneo associato a (11.9); ne segue la prima parte del lemma.

(18) Cfr. il lemma 1.3 di [33]. 
La dimostrazione della seconda parte del lemma, che qui per brevità omettiamo, si ottiene poi ripetendo i ragionamenti tenuti nel corso della dimostrazione del lemma 5.3 di [33] per stabilire l'analogo risultato nel caso ellittico.

Drmostrazione del teorema 8.3. - Osserviamo che, nelle ipotesi poste, dal lemma 11.1 segue che gli operatori $A^{*}$ e $B_{i j}^{\prime}$ verificano le ipotesi $\left.\mathscr{H}_{1}\right)-\mathscr{H}_{4}$ ), $\left.\left.\mathscr{H}_{6}\right), \mathscr{H}_{i}\right)$ con $m^{(i)}-v_{i}$ in luogo di $v_{i} \theta$ l'ipotesi $\mathcal{H}_{5}$ ) con $-\theta$ in luogo di $\theta$.

Se ne deduce che, fissato $s$, esiste una costante $\lambda_{0}>0$ tale che per ogni $\lambda$, con $|\lambda| \geq \lambda_{0}$ e $\arg \lambda=\theta$, i problemi (8.15)-(8.17) e (11.9) ammettono ciascuno al più una soluzione.

Da tali considerazioni, da noti risultati relativi agli operatori lineari e dal lemma 11.3 si deduce la tesi nell' ulteriore ipotesi che $\sigma \in C^{\infty}(\bar{\Omega}-S)$.

Se poi $\sigma \notin C^{\infty}(\bar{\Omega}-S)$, consideriamo il problema $(8.15)-(8.17)$ con $\sigma_{k}$ in luogo di $\sigma$ (cfr. l'ipotesi $\left.\mathscr{H}_{8}\right)$ ).

Per quanto sopra stabilito e per il teorema $8.2 \mathrm{si} \mathrm{ha}$ evidentemente che esiste una costante $\lambda_{0}>0$, indipendente da $k$, tale che per ogni $\lambda$, con $|\lambda| \geq \lambda_{0}$ e $\arg \lambda=\theta$, detto problema ammette un' unica soluzione $u_{k}$ la quale soddisfa la limitazione

$$
\left\|u_{k}\right\|_{W_{s}^{m}(\Omega)} \leq c\|f\|_{s+\chi}^{2}-m(\Omega)
$$

con $c$ costante indipendente da $k$.

Tenuto conto del lemma 7.6, dalla (11.11) segue che da $\left|u_{k}\right|$ si può estrarre una successione debolmente convergente in $W_{s}^{m}(\Omega)$ e fortemente convergente in $W_{\beta}^{j}(\Omega)$, per ogni $j \in J(\mathscr{K})$ e $<m$ e per ogni $\beta>s+j-m$, verso una funzione $u \in W_{s}^{m}(\Omega)$, la quale, evidentemente, riesce una soluzione del problema (8.15)-(8.17). Ne segue la tesi.

\section{CAPITOLO III.}

\section{Uiteriori risultati relativi al problema di Dirichlet per le equazioni fortemente quasi-ellittiche in un dominio limitato.}

\section{2. - Lemmi preliminari.}

Nel seguito considereremo operatori differenziali lineari della forma (8.1) e fortemente quasi-ellittici. Pertanto $\left({ }^{19}\right)$, supporremo che gli interi $m_{1}, \ldots, m_{n}$

(19) Ofr. quanto osservato nella parte finale del n. 8 . 
siano pari e porremo

$$
m_{k}=2 N_{k} \quad(k=1, \ldots, n), \quad m^{(i)}=2 N^{(i)} \quad(i=1, \ldots, t), \quad m=2 N .
$$

Indicheremo: con $W_{s}^{2 N}\left(\Omega, \partial / \hat{c} \mathbf{n}_{i}\right), s$ reale, la classe delle funzioni $u \in W_{s}^{2 N}(\Omega)$ e soddisfacenti le condizioni

$$
\frac{\partial^{j} u}{\partial \mathbf{n}_{i}^{j}}=0 \quad \text { su } \quad \partial_{i} \Omega, \quad j=0, \ldots, N^{(i)}-1, \quad i \in \mathfrak{g}
$$

con $\mathscr{C}^{\infty}\left(\bar{\Omega}, \partial / \partial \mathbf{n}_{i}\right)$ la classe delle funzioni $u \in \mathscr{C}^{\infty}(\bar{\Omega})$ e soddisfacenti le (12.1).

Lemma 12.1. - Supponiamo che sia verificata l'ipotesi $a_{0}$ ) e che esista una funzione $\sigma(x) \in C^{\infty}(\bar{\Omega}-S) \cap C^{0,1}(\bar{\Omega})$ e soddisfacente la $(8.10)$.

Allora, per ogni s reale, $\check{C}^{\infty}\left(\bar{\Omega}, \partial / \hat{c} \mathbf{n}_{i}\right)$ è denso in $W_{s}^{2 N}\left(\Omega, \partial / \hat{c} \mathbf{n}_{i}\right)$.

Infatti, assegnamo $A$ e $\lambda$ tali che il problema (8.24) sia univocamente risolubile per ogni $f \in L_{s+\chi}^{2}-2 N(\Omega)$ (cfr. il corollario 8.1).

Inoltre, assegnata $u \in W_{s}^{2 N}\left(\Omega, \partial / \partial \mathbf{n}_{i}\right)$, poniamo

$$
f=A u+\lambda \sigma^{-\chi} u
$$

e indichiamo con $\left\{f_{k}\right\}$ una successione di funzioni di classe $C^{\infty}(\bar{\Omega}) \cap L_{s+\chi-2 N}^{2}(\Omega)$ convergente ad $f$ in $L_{s+\chi_{-2 N}}^{2}(\Omega)$.

Indichiamo inoltre, per ogni $k$, con $u_{k}$ la soluzione del problema

$$
\begin{aligned}
& \boldsymbol{u}_{k} \in W_{s}^{2 N}\left(\Omega, \partial / \partial \mathbf{n}_{i}\right), \\
& A u_{k}+\lambda \sigma^{-} \boldsymbol{u}_{k}=f_{k} \text { in } \Omega .
\end{aligned}
$$

Evidentemente risulta

$$
\lim _{k}\left\|u_{k}-u\right\|_{W_{s}^{2 N}(\Omega)}^{2 N}=0 .
$$

Inoltre dal corollario 5.2 segue che $u_{k} \in O^{\infty}(\bar{\Omega}-S)$.

Si ottiene allora la tesi osservando che per il lemma 7.7 si ha per ogni $k$

$$
\lim _{\varepsilon \rightarrow 0}\left\|\delta_{\varepsilon} u_{k}-u_{k}\right\|_{W_{s}^{2 N}(\Omega)}=0
$$

e che le funzioni $\delta_{\varepsilon} u_{k} \in C^{\circ}\left(\bar{\Omega}, \partial / \partial \mathbf{n}_{i}\right)$. 
Facendo ora uso delle notazioni introdotte per il lemma 9.1, supponiamo che l'operatore $A$ definito dalla (9.1) verifichi l'ipotesi $\mathfrak{G}_{1}$ ) e la seguente ipotesi :

G्2) $A$ è fortemente quasi-ellittico in $\overline{Q_{T_{0}}}$, cioè riesce

$$
\operatorname{Re} A_{0}(x, \xi)=R e \underset{<\mu, q>=2 N}{\mathrm{\Sigma}} a_{\mu}(x) \xi^{\mu}>0 \quad \forall x \in \overline{Q_{r_{0}}} \text { } \Theta \forall \xi \in R^{n}-\{0\} .
$$

Indichiamo con $\mathcal{Q}\left(\boldsymbol{\Sigma}_{r_{0}}\right)$ la classe delle funzioni $u \in W^{2 N}\left(\boldsymbol{\Sigma}_{r_{0}}\right)$ e tali che

$$
\frac{\partial u}{\partial x_{n}^{j}}=0 \quad \text { su } \quad \sigma_{r_{0}}, \quad j=0, \ldots, N_{n}-1
$$

e con $\mathcal{Q}\left(\Gamma_{r_{0}}\right)$ lo spazio $W^{2 N}\left(\Gamma_{r_{0}}\right)$

Lemma 12.2. - Se sono verificate le ipotesi $\left.\mathscr{G}_{1}\right)$ e $\left.\mathscr{G}_{2}^{\prime}\right)$, per ogni $u \in \mathscr{Q}\left(Q_{r_{0}}\right)$ e per ogni $\lambda>0$ sussiste la limitazione

$$
\begin{aligned}
& \underset{<\alpha, q>=2 N}{\Sigma}\left|D^{\alpha} \boldsymbol{u}\right|_{2, Q_{r}}+\lambda^{1 / 2} \underset{<x, q>=N}{\Sigma}\left|D^{\alpha} \boldsymbol{u}\right|_{2, Q_{r}}+\lambda|\boldsymbol{u}|_{2, Q_{r}} \\
& \leq c\left(|A u+\lambda u|_{2, Q_{r_{0}}}+|\boldsymbol{u}|_{2, Q_{r_{0}}}\right),
\end{aligned}
$$

dove $r<r_{0}$ e dove c è una costante indipendente da $u$ e $\lambda$.

Infatti, poniamo per ogni $x^{k} \in \bar{Q}_{r_{0}}$

$$
A_{0 k}=\underset{<\mu, q>=2 N}{\Sigma} a_{\mu}\left(x^{k}\right) D^{\mu}
$$

Per la compattezza di $\bar{Q}_{r_{0}}$ e per la continuità in $\bar{Q}_{r_{0}}$ dei coefficienti $a_{\mu}(x)$ per $\langle\mu, q\rangle=2 N$, in corrispondenza di $\varepsilon>0$ esiste un insieme finito $\left\{\left.x^{k}\right|_{k=1} ^{p}\right.$ di punti di $\bar{Q}_{r_{0}}$ godenti della proprietà che ogni $x^{k}$ ha un intorno $n$-dimensionale $\mathfrak{d}\left(x^{k}\right)$ tale che $\left\{\mathfrak{S}\left(x^{k}\right)\right\}_{k=1}^{p}$ sia un ricoprimento di $\bar{Q}_{r_{0}}$ e tale inoltre che si abbia

$$
\left|A_{0} v-A_{0 k} v\right|_{2, Q_{r_{0}}} \leq \varepsilon \sum_{<\alpha, q>=2 N}^{\Sigma}\left|D^{\alpha} v\right|_{2, Q_{r_{0}}}
$$

per ogni $v \in W^{2 N}\left(Q_{r_{0}}\right)$ ed a supporto compatto in $\overline{Q_{r_{0}}} \cap \mathfrak{I}\left(x^{k}\right)$.

Indicheremo con $\left(\varphi_{k}\right\}_{k=1}^{p}, \varphi_{k} \in C_{0}^{\infty}\left(R^{n}\right)$ e supp $\varphi_{k} \subset \mathfrak{J}\left(x^{k}\right)$, una partizione dell' unità su $\bar{Q}_{r_{0}}$ relativa al suddetto ricoprimento. 
Sia ora $u$ una funzione di classe $\mathscr{Q}\left(Q_{r_{0}}\right)$ e sia $\zeta(x)$ la funzione definita nel corso della dimostrazione del lemma 9.1. Poniamo $v=\zeta u$ e $v_{k}=\varphi_{k} v$.

Indicheremo con $c_{1}, \ldots, c_{9}$ delle costanti positive indipendenti da $u, r, r^{\prime}$ e $\lambda$. Con noti procedimenti si prova che riesce

$$
R e \int_{Q_{\tau_{0}}} A_{0 k} v_{k} \cdot \bar{v}_{k} d x \geq c_{1} \underset{<\alpha, q>=N}{\Sigma}\left|D^{\alpha} v_{k}\right|_{2, Q_{\tau_{0}}}^{2} .
$$

Consegue che per ogni $\lambda>0$ si ha

$$
\begin{gathered}
\sum_{<\alpha, q>=N}\left|D^{\alpha} v_{k}\right|_{2, Q_{r_{0}}}^{2}+\lambda\left|v_{k}\right|_{2, Q_{r_{0}}}^{2} \leq c_{2} \int_{Q_{r_{0}}}\left|\left(A_{0 k} v_{k}+\lambda v_{k}\right) \cdot v_{k}\right| d x \\
\leq \frac{c_{3}}{\lambda}\left|A_{0 k i} v_{k}+\lambda v_{k}\right|_{2, Q_{r_{0}}}^{2}+\frac{\lambda}{2}\left|v_{k}\right|_{2, Q_{r_{0}}}^{2}
\end{gathered}
$$

e quindi si ha anche

$$
\lambda^{1 / 2} \sum_{<\alpha, q>=N}\left|D^{\alpha} v_{k}\right|_{2, Q_{r_{0}}}+\lambda\left|v_{k}\right|_{2, Q_{r_{0}}} \leq c_{4}\left|A_{0 k} v_{k}+\lambda v_{k}\right|_{2, Q_{r_{0}}} .
$$

Dalle (12.5), (12.7) e dal lemma 7.1 segue evidentemente che riesce

$$
\begin{gathered}
\lambda_{</ 2} \underset{<\alpha, q>=N}{\sum}\left|D^{\alpha} v_{k}\right|_{2, Q_{r_{0}}}+\lambda\left|v_{k}\right|_{2, Q_{r_{0}}} \leq c_{5}\left(\left|A v_{k}+\lambda v_{k}\right|_{2, Q_{r_{0}}}\right. \\
\quad+\varepsilon \sum_{<\alpha, q>=2 N}\left|D^{\alpha} v_{k}\right|_{2, Q_{r_{0}}}+c(\varepsilon)\left|v_{k}\right|_{2, Q_{r_{0}}} .
\end{gathered}
$$

Inoltre dalle (12.8) e (9.3) si ha

$$
\begin{aligned}
\sum_{<\alpha, q>=2 N}\left|D^{\alpha} v_{k}\right|_{2, Q_{r_{0}}} \leq c_{6}\left(\left|A v_{k}+\lambda v_{k}\right|_{2, Q_{r_{0}}}\right. \\
\left.+\varepsilon \sum_{<\alpha, q>=2 N}\left|D^{\alpha} v_{k}\right|_{2, Q_{r_{0}}}+(1+c(\varepsilon))\left|v_{k}\right|_{2, Q_{r_{0}}}\right)
\end{aligned}
$$

da tale relazione e dalla (12.8) si deduce la limitazione

$$
\begin{gathered}
\sum_{<\alpha, q>=2 N}\left|D^{\alpha} v_{k}\right|_{2,} Q_{r_{0}}+\lambda^{1 / 2} \sum_{<\alpha, q>=N}\left|D^{\alpha} v_{k}\right|_{2,} Q_{r_{0}}+\lambda\left|v_{k}\right|_{2, Q_{r_{0}}} \\
\leq c_{7}\left(\left|A v_{k}+\lambda v_{k}\right|_{2, Q_{r_{0}}}+\left|v_{k}\right|_{2, Q_{r_{0}}}\right) \\
\leq c_{B}\left(|A(\zeta u)+\lambda \zeta u|_{2, Q_{r_{0}}}+\sum_{<\mu, q><2 N}\left|D^{\alpha}(\zeta u)\right|_{2, Q_{r_{0}}}\right) .
\end{gathered}
$$


Tenuto conto che $\zeta u=v=\sum_{k=1}^{p} v_{k}$, da quest'ultima relazione e dal lemma 7.1 segue che si ha

$$
\begin{aligned}
& \sum_{<\alpha, q>=2 N}\left|D^{\tilde{\alpha}}(\zeta u)\right|_{2, Q_{r_{0}}}+\lambda^{1 / 2} \sum_{<\alpha, q>=N}\left|D^{\alpha}(\zeta u)\right|_{2, Q_{r_{0}}}+\lambda|\zeta u|_{2, Q_{r_{0}}} \\
& \leq c_{9}\left(|A(\zeta u)+\lambda \zeta u|_{2, Q_{r_{0}}}+|\zeta u|_{2, Q_{r_{0}}}\right) \text {. }
\end{aligned}
$$

Dalla (12.9) si deduce la tesi con considerazioni analoghe a quelle tenute per dedurre dalle (9.6) e (9.7) la tesi del lemma 9.1.

Assegnato ora in $\Omega$ un operatore della forma (8.1), dimostriamo il segnente

Lemma 12.3. - Supponiamo che sia verificata l'ipotesi $a_{0}$ ), che $i$ coefficienti $a_{\mu}(x)$ verifichino $l^{\prime}$ ipotesi $\left.\mathfrak{H}_{1}\right)$ e che sia soddisfatta la condizione $(8.23)$ di forte quasi-ellitticità.

Allora, assegnati $i$ numeri reali $\beta \leq \chi$, s e una funzione $\sigma(x) \in C^{0,1}(\bar{\Omega})$ e soddisfacente la (8.10), per ogni $u \in W_{s+2 N}^{2 N}\left(\Omega, \partial / \partial \boldsymbol{n}_{i}\right)$ e per ogni $\lambda>0$ sussiste la limitazione

$$
\begin{gathered}
\sum_{<\alpha, q>\leq 2 N}\left|D^{\alpha} u\right|_{2, s+<\alpha, \tau>}+\lambda^{1 / 2} \sum_{<\alpha, q>=N}\left|D^{\alpha} u\right|_{2, s+<\alpha, \tau>+(\chi-\beta) / 2} \\
\quad+\lambda|u|_{2, s+\chi-\beta} \leq c\left(\left|A u+\lambda \sigma^{-\beta} u\right|_{2, s+\chi}+|u|_{2, s}\right)
\end{gathered}
$$

dove c è una costante indipendente da $u$ e $\lambda$.

Infatti, poniamo $\left({ }^{20}\right)$

$$
\tilde{A}^{(\beta)}=\left(\frac{\sigma^{*}\left(x_{0}, \xi\right)}{\rho\left(x_{0}\right)}\right)^{\beta} \tilde{A}
$$

dove $\tilde{A}$ è l'operatore definito dalla (8.8).

Con considerazioni analoghe a quelle tenute per dedurre dal lemma 9.1 la (9.15) e dal lemma 10.1 la (10.5), si deduce dal lemma 12.2 che si ha per $u \in W_{s+2 N}^{2 N}\left(\Omega, \partial / \partial n_{i}\right)$ e $\lambda>0$

$$
\begin{aligned}
& \sum_{<\alpha, q>=2 N}\left|D^{\alpha} u^{*}\right|_{\left.2, I_{1 / 2}^{*} / x_{0}\right)}+\lambda^{1 / 2} p(\chi-\beta) / 2\left(x_{0}\right) \sum_{<\alpha, q>=N}\left|D^{\alpha} u^{*}\right|_{2, I_{1 / 2}^{*}\left(x_{0}\right)} \\
& +\lambda_{p} \chi-\beta\left(x_{0}\right)\left|u^{*}\right|_{2, I_{1 / 2}^{*}\left(x_{0}\right)} \leq c_{1}\left(\left|\tilde{A}^{(\beta)} u^{*}+\lambda_{\rho} \chi-\beta\left(x_{0}\right) u^{*}\right|_{2, I^{*}\left(x_{0}\right)}+\left|u^{*}\right|_{2, I^{*}\left(x_{0}\right)}\right),
\end{aligned}
$$

dove $c_{1}$ è una costante indipendente da $u, \lambda$ e $x_{0}$.

(20) Si tenga presente l'osservazione 8.1. 
Operando in (12.11) il cambiamento di variabili (7.6) si ha

$$
\begin{aligned}
& \sum_{<\alpha, q>=2 N} \rho^{<\alpha, \tau>-|\tau| / 2}\left(x_{0}\right)\left|D^{\alpha} u\right|_{2, I_{1 / 2}\left(x_{0}\right)}+\lambda^{1 / 2} \sum_{<\alpha, q>=N^{N}} \rho^{<\alpha, \tau>+(\chi-\beta) / 2-|\tau| / 2}\left(x_{0}\right)\left|D^{\alpha} u\right|_{2, I_{1 / 2}\left(x_{0}\right)} \\
& +\lambda \rho^{x-\beta-|\tau| / 2}\left(x_{0}\right)|u|_{2, I_{1 / 2}\left(x_{0}\right)} \leq c_{1}\left(\rho^{\chi-\beta-|\tau| / 2}\left(x_{0}\right)\left|\sigma^{\beta} A u+\lambda u\right|_{2,1\left(x_{0}\right)}\right. \\
& \left.+p^{-|\tau| / 2}\left(x_{0}\right)|u| 2, I\left(x_{0}\right)\right) \\
& \leq c_{2}\left(\rho^{X-|\tau| / 2}\left(x_{0}\right)\left|A u+\lambda \sigma^{-\beta} u\right|_{2, I\left(x_{0}\right)}+\rho^{-|\tau| / 2}\left(x_{0}\right)|u|_{2, I\left(x_{0}\right)}\right),
\end{aligned}
$$

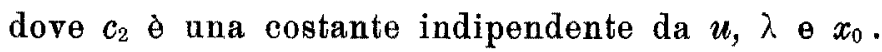

Da quest' nltima relazione si deduce la tesi con ragionamenti analoghi a quelli tenuti per dedurre dalla (9.18) la tesi del teorema 8.1.

Nel seguito supporremo che sia

$$
\tau_{k}=q_{k}, \quad k=1, \ldots, n,
$$

e indicheremo con $H_{0, s}^{N}(\Omega)$, s reale, la chiusura di $C_{0}^{\infty}(\Omega)$ in $W_{s}^{N}(\Omega)$.

Inoltre considereremo la seguente ipotesi $\left({ }^{21}\right)$ :

$\left.a_{1}\right)$ Esiste una fanzione $\sigma(x) \in C^{\infty}(\bar{\Omega}-S) \cap C^{0,1}(\bar{\Omega})$, soddisfacente la (8.10) e le limitazioni

$$
\left|D^{\alpha} \sigma(x)\right| \leq c_{\alpha} \sigma^{-|\alpha|+1}(x) \quad \forall x \in \Omega
$$

dove le $c_{x}$ sono delle costanti indipendenti da $x$.

Assegnamo ora una forma sesquilineare e continua su $W_{s}^{N}(\Omega) \times W_{s}^{N}(\Omega)$ del tipo

$$
\alpha_{s}(\boldsymbol{u}, v)=\int_{\substack { \Omega \\
\begin{subarray}{c}{\langle h, q>\leq N \\
\langle k, q>\leq N{ \Omega \\
\begin{subarray} { c } { \langle h , q > \leq N \\
\langle k , q > \leq N } }\end{subarray}} a_{k k}(x) D^{h} \boldsymbol{u} \cdot \overline{D^{k}\left(\sigma^{2 s} v\right)} d x
$$

dove $\sigma(x)$ è la funzione definita nell'ipotesi $a_{1}$ ), supponendo che sia verificata la condizione di forte quasi-ellitticità :

$$
R e \sum_{<h, q>=<k, q>=N} a_{h k}(x) \xi^{\alpha+\beta}>0 \quad \forall x \in \bar{\Omega} \text { e } \forall \xi \in R^{n}-\{0\} .
$$

(21) In conseguenza del lemma 3.1 del cap. 6 di J. NEČs [26], si ha che tale ipotesi è, ad es., verificata nel caso in cui $Q$ ha frontiera "localmente lipschitziana e $S=\partial Q$, e nel caso in cui $t=\mathbf{2}, S^{(1)}=\partial Q^{(1)}, S^{(2)}=\emptyset$ e $\mathbf{Q}^{(1)}$ ha frontiera "localmente lipschitziana*. 
Si ha:

Lema 12.4. - Siano verificate le ipotesi $\left.a_{0}\right)$ e $\alpha_{1}$ ), la condizione (12.15) di forte quasi-ellitticità ed inollire $i$ coefficienti $a_{h k}(x)$ siano di classe $C^{0}(\bar{\Omega})$ per $<h, q\rangle=\langle k, q\rangle=N$, di classe $L^{\infty}(\Omega)$ per $\langle h+k, q\rangle \geq \beta$ e di classe $L_{\beta-<h+k, q>(\Omega)}^{\infty}$ per $<h+k, q><\beta$, dove $\beta$ è un fissato numero reale appartenente all' intervallo $[0,2 N]$.

Allora, assegnato $s_{0}>0$, per ogni $s \in\left[-s_{0}, s_{0}\right]$ e per ogni $u \in H_{0, s}^{N}(\Omega)$ si ha

$$
\begin{gathered}
\sum_{<\alpha, q>\leq N}\left|D^{\alpha} \boldsymbol{u}\right|_{2, s}^{2} \leq c \text { Re } a_{s}(u, u) \\
+c_{0}\left(s^{2} \sum_{<\alpha, q><N}\left|D^{\alpha} \boldsymbol{u}\right|_{2, s+<\alpha, q>-N}^{2}+|\boldsymbol{u}|_{2, s-\beta / 2}^{2}\right),
\end{gathered}
$$

dove $c$ e co sono due costanti positive indipendenti da $u$ es.

Se non è verificata l'ipotesi $\left.a_{1}\right)$, la (12.16) sussiste per $s=0$.

E sufficiente stabilire la (12.16) per funzioni $u \in C_{0}^{\infty}(\Omega)$.

Poniamo

$$
\begin{gathered}
a_{s}^{\prime}(u, v)=\int_{\Omega} \sum_{<h+k, q>\geq 3} a_{k k}(x) D^{h} u \cdot \overline{D^{k}\left(\sigma^{2 s} v\right)} d x \\
a_{s}^{\prime \prime}(u, v)=a_{s}(u, v)-a_{s}^{\prime}(u, v) .
\end{gathered}
$$

Assegnamo $s \in\left[-s_{0}, s_{0}\right]$ e $u \in C_{0}^{\infty}(\Omega)$.

Nel corso di questa dimostrazione indicheremo con $c_{1}, \ldots, c_{8}$ delle costanti positive indipendenti da $u$ e $s$.

Notoriamente, nelle ipotesi poste si ha (cfr. E. Giusmi [16])

$$
\operatorname{Re} a_{0}^{\prime}(u, u) \geq c_{1} \underset{<\alpha, q>\leq N}{\Sigma}\left|D^{\alpha} u\right|_{2,0}^{2}-c_{2}|u|_{2,0}^{2}
$$

D'altra parte osserviamo che riesce

$$
a_{s}^{\prime}(u, u)=a_{0}^{\prime}\left(\sigma^{s} u, \sigma^{s} u\right)+B_{s}(\boldsymbol{u})
$$

dove

$$
\begin{gathered}
B_{s}(u)=\sum_{<h+k, q>\geq \beta} \int_{\Omega} a_{h k}(x)\left(\sum_{\alpha<k} \sum_{\gamma \leq \alpha}\left(\begin{array}{l}
k \\
\alpha
\end{array}\right)\left(\begin{array}{l}
\alpha \\
\gamma
\end{array}\right) D^{k} u \cdot \overline{D^{k-\alpha} \sigma^{s} \cdot D^{\alpha-\gamma} \sigma^{s} \cdot D^{\Upsilon} u}\right. \\
\left.-\sum_{\alpha<h}\left(\begin{array}{l}
h \\
\alpha
\end{array}\right) D^{h-\alpha \sigma^{s}} \cdot D^{\alpha} u \cdot \overline{D^{k}\left(\sigma^{s} u\right)}\right) d x .
\end{gathered}
$$


Tenuto conto delle $(8.10),(12.13)$ ed osservando che

$$
|h-\alpha| \leq N-<\alpha, q>\text { per } \alpha \leq h \text { e } \quad<h, q>\leq N \text {, }
$$

si verifica facilmente che riesce

$$
\left|B_{s}(\boldsymbol{u})\right| \leq c_{3}|\boldsymbol{s}|\left(\sum_{<\alpha, q><N}\left|D^{\alpha} u\right|_{2, s+<\alpha, q>-N)} \cdot \sum_{<h, q>\leq N}\left(\left|D^{h} u\right|_{2, s}+\left|D^{h}\left(\sigma^{s} u\right)\right|_{2,0}\right) \cdot\right.
$$

Analogamente si verifica che si ha per $\langle h, q\rangle \leq N$

$$
\left|D^{h}\left(\sigma^{s} \boldsymbol{u}\right)-\sigma^{s} D^{h} u\right|_{2,0} \leq c_{4}|\boldsymbol{s}|_{<x, q><N}\left|D^{\alpha} u\right|_{2, s+<\alpha, q>-N} .
$$

D'altra parte per la (12.17) si ha

$$
\operatorname{Re} \alpha_{0}^{\prime}\left(\sigma^{s} u, \sigma^{s} u\right) \geq c_{1} \underset{<h, q>\leq N}{\Sigma}\left|D^{h}\left(\sigma^{s} u\right)\right|_{2,0}^{2}-c_{2}\left|\sigma^{s} u\right|_{2,0}^{2} .
$$

Dalle (12.18)-(12.22) si deduce evidentemente la limitazione

$$
\begin{gathered}
\sum_{<x, q>\leq N}\left|D^{\alpha} u\right|_{2, s}^{2} \leq c_{5} \operatorname{Re} \alpha_{s}^{\prime}(u, u) \\
+c_{6}\left(s_{<}^{2} \sum_{<\alpha, q><N}\left|D^{\alpha} u\right|_{2, s+<\alpha, q>-N}^{2}+|u|_{2, s}^{2}\right) .
\end{gathered}
$$

Se $\beta=0$, la (12.23) coincide con la $(12.16)$ e quindi si ha la tesi.

Se $\beta>0$, osserviamo che riesce

$$
\left|a_{s}^{\prime \prime}(\boldsymbol{u}, u)\right| \leq c_{7} \sum_{<h+k, q><\beta} \sum_{\alpha \leq k} \int_{\Omega}\left|a_{h k} D^{h} u \cdot \sigma^{2 s-|k-\alpha|} \cdot D^{\alpha} u\right| d x
$$

Tenuto conto che per ipotesi $a_{h k}(x) \in L_{\beta}^{\infty}<k+k, q>(\Omega)$ per $<h+k, q><\beta$ ed osservando che

$$
2 s-|k-\alpha|-\beta+<h+k, q>\geq 2 s+<h, q>+<\alpha, q>-\beta,
$$


dalla (12.24) si deduce facilmente la limitazione

$$
\left|\boldsymbol{a}_{s}^{\prime \prime}(\boldsymbol{u}, \boldsymbol{u})\right| \leq c_{3} \sum_{<h, q>\leq N}\left|D^{h} \boldsymbol{u}\right|_{2, s+<h, q>-\beta / 2} \cdot \underset{<\alpha, q><N}{\Sigma}\left|D^{\alpha} \boldsymbol{u}\right|_{2, s+<\alpha, q>-\beta / 2} \cdot
$$

Dalle (12.23), (12.25) e dal lemma 7.5 si deduce la (12.16), e cioè la tesi, con note considerazioni.

Indicheremo con $W_{0, s}^{N}(\Omega), s$ reale, la classe delle funzioni $u \in W_{s}^{N}(\Omega)$ e soddisfacenti le (12.1).

Evidentemente $\left.{ }^{22}\right)$ riesce $H_{0, s}^{N}(\Omega) \subseteq W_{0, s}^{N}(\Omega)$. Vogliamo inoltre dimostrare che

$\mathrm{L}_{\text {EMMA }}$ 12.5. - Se sono verificate le ipotesi $\left.a_{0}\right)$ e $\left.a_{1}\right)$, si ha $H_{0, s}^{N}(\Omega)=W_{0, s}^{N}(\Omega)$.

Dobbiamo solo dimostrare che $w \in W_{0, s}^{N}(\Omega)$ implica $w \in H_{0, s}^{N}(\Omega)$.

Assegnamo un operatore differenziale lineare $A$ della forma

$$
A u=\sum_{\substack{<, i, q>\leq N \\\langle k, q>\leq N}} D^{k}\left(a_{h l}(x) D^{k} u\right)
$$

a coefficienti $a_{h k}(x) \in C^{\infty}(\bar{\Omega})$ e soddisfacente la condizione (12.15) di forte quasiellitticità.

Dai lemmi 12.4 e 7.5 segue che esistono delle costanti positive $c, c_{0}$ e $c_{0}^{\prime}$ tali che si ha per ogni $u \in H_{0, s}^{N}(\Omega)$

$$
\begin{gathered}
\operatorname{Re} \alpha_{s}(u, u) \geq c\|u\|_{W_{s}^{N}(\Omega)}^{2}-c_{0}\|u\|_{L_{s-N}^{2}(\Omega)}^{2} \\
\geq c\|u\|_{W_{s}}^{2}(\Omega) \\
-c_{0}^{\prime} \int_{\Omega} \sigma^{-2 N} u \cdot \overline{\sigma^{2 s} u} d x
\end{gathered}
$$

dove $\alpha_{s}(u, v)$ è definita dalla (12.14).

Fissiamo un numero complesso $\lambda$ tale che $R e \lambda \geq c_{0}^{\prime}$ e tale inoltre che per ogni $f \in L_{s+N}^{2}(\Omega)$ il problema

$$
\begin{aligned}
& u \in W_{s+N}^{2 N}\left(\mathbf{\Omega}, \partial / \hat{\mathfrak{c}} \mathbf{n}_{i}\right) \\
& A u+\lambda \sigma^{-2 N} u=f \text { in } \Omega
\end{aligned}
$$

sia univocamente risolubile (cfr. il corollario 8.1).

(22) Si tengono presenti il lemma 1.7 o la nota (13). 
Assegnata ora $w \in W_{0, s}^{N}(\Omega)$, osserviamo che

$$
a_{s}(w, v)+\lambda \int_{\Omega} \sigma^{-2 N} w \cdot \overline{\sigma^{2 s} v} d x
$$

riesce un funzionale lineare e continuo su $H_{0, s}^{N}(\Omega)$, e quindi esiste $u_{0} \in H_{0, s}^{N}(\Omega)$ tale che

$$
a_{s}\left(w-u_{0}, v\right)+\lambda \int_{\Omega} \sigma^{-2 N}\left(w-u_{0}\right) \cdot \overline{\sigma^{2 s} v} d x=0 \quad \forall v \in H_{0, s}^{N}(\Omega) .
$$

Dal corollario 5.2 si deduce, con note considerazioni, che $w-u_{0} \in C^{\infty}(\bar{\Omega}-S)$, e quindi per il teorema 8.1 si ha anche che $w-u_{0}$ è una soluzione del problema amogeneo associato a (12.28). Ne segue che risulta $w=u_{0} \in H_{0, s}^{N}(\Omega)$ e quindi si ha la tesi.

\section{3. - Teoremi di unicità.}

I teoremi di unicita e di esistenza per il problema di Dirichlet che ci proponiamo di stabilire in quest' ultima parte del lavoro, si applicano quando $A$ risulta fortemente quasi-ellittico in $\bar{\Omega}$, tale cioè che si abbia

$$
R e \underset{<\mu, q>=2 N}{\Sigma} a_{\mu}(x) \xi^{\mu}>0 \quad \forall x \in \bar{\Omega} \text { e } \forall \xi \in R^{n}-\{0\},
$$

e quando sono verificate le ipotesi $\left.a_{0}\right), a_{1}$ ) e la seguente condizione:

$\left.a_{2}\right) t \leq 2, N=N^{(1)}, \Omega^{(1)}$ ha frontiera «localmente lipsehitziana»e, se $t=2, S^{(2)}=\varnothing$.

Nel seguito $\sigma(x)$ denoterà la funzione definita dall' ipotesi $a_{1}$ ).

Cominciamo col dimostrare il seguente teorema di unicità:

TeORema 13.1. - Supponiamo che siano verificate le ipotesi $\left.\left.a_{0}\right), a_{1}\right)$ e $a_{2}$ ), che $A$ sia fortemente quasi-ellitlico in $\bar{\Omega}$ e che $i$ coefficienti $a_{\mu}(x)$ siano di classe $C^{0}(\bar{\Omega})$ per $\langle\mu, q\rangle=2 N$, di classe $L_{N+\gamma-<\mu, q>}^{\infty}(\Omega)$ per $\langle\mu, q><2 N$, dove $\gamma$ è un fissato numero reale tale che $\sup _{<\alpha, q><2 N}<\alpha, q>-N \leq \gamma \leq N$.

Esistono due costanti $\lambda_{0}>0$ e $\left.\eta_{0} \in\right] 0,1 / 2\left[\right.$ lali che per ogni $\lambda \geq \lambda_{0}$, per ogni $s \in\left[N-\eta_{0}, N+\eta_{0}\right]$ e per ogni $u \in W_{s}^{2 N}\left(\Omega, \partial / \partial n_{i}\right)$ sussiste la limitazione

$$
\|u\|_{W_{s}^{2 N}(\Omega)} \leq c\left\|A u+\lambda \sigma^{-2} \gamma u\right\|_{L_{s}^{2}(\Omega)},
$$

dove $c$ è una costante indipendente da $u$, s e $\lambda\left({ }^{23}\right)$.

(23) Osserviamo, come si deduce dalla dimostrazione, che se non è verificata l'ipotesi $\left.a_{1}\right)$ la (13.2) sussiste per $s=0$ e per ogni funzione $\sigma(x) \in C^{0,1}(\overline{\mathrm{g}})$ e soddisfacente la (8.10). 
Premettiamo il seguente

LEMMA 13.1. - Sia verificata l'ipotesi $a_{2}$ ) e sia $s_{0}$ un assegnato numero reale $<1 / 2$.

Per ogni $s \leq s_{0}$ e per ogni $u \in H_{0, s}^{N}(\Omega)$ si ha

$$
|\boldsymbol{u}|_{2, s-N} \leq c \sum_{<\alpha, q>=N}\left|D^{x} \boldsymbol{u}\right|_{2, s},
$$

dove c è una costante indipendente da $u$ e $s$.

E evidentemente sufficiente stabilire la $(13.3)$ per funzioni $u \in C_{0}^{\infty}(\Omega)$.

Allora se $t=1$, osservando che in tal easo risulta $\langle\alpha, q\rangle=|\alpha|$, la tesi. consegue dall'osservazione 7.3 e dal lemma 7.9 .

Se $\iota=2$, ancora dall' osservazione 7.3 e dal lemma 7.9 segue che, fissato $y \in \mathbf{\Omega}^{2}$, si ha per $s \leq s_{0}$

$$
\int_{\Omega^{(1)}}\left|\rho^{s-N^{(1)}}(x, y) u(x, y)\right|^{2} d x \leq c \underset{|\alpha|=N^{(1)}}{\Sigma} \int_{\Omega^{(1)}}\left|\rho^{s}(x, y) D_{x}^{\alpha} u(x, y)\right|^{2} d x
$$

dove $c$ è una costante indipendente da $u, s$ e $y$. Siccome per ipotesi $N=N^{(1)}$, da quest' ultima relazione si deduce la (13.3) e quindi si ha la tesi.

Dimostrazione del teorema 13.1. - Procedendo come nella dimostrazione del lemma 12.2, consideriamo, in corrispondenza di $\varepsilon>0$, un ricoprimento finito di $\bar{\Omega}$ mediante gli intorni $\mathfrak{J}\left(x^{k}\right), k=1, \ldots, p$, in modo che si abbia

$$
\left|A_{0} v-A_{0 k s} v\right|_{2, s} \leq \varepsilon \sum_{<x, q>=2 N}^{\Sigma}\left|D^{\alpha} v\right|_{2, s}
$$

per ogni $v \in W_{s}^{2 N}(\Omega)$ ed a supporto compatto in $\bar{\Omega} \cap \mathfrak{J}\left(x^{\bar{k}}\right)$, e una partizione dell' unità $\left\{\varphi_{k}(x)\right\}_{k=1}^{p}$ su $\bar{\Omega}$ relativa a tale ricoprimento, con $\varphi_{k} \in C_{0}^{\infty}\left(R^{n}\right)$ $\theta \operatorname{supp} \varphi_{k} \subset \mathfrak{d}\left(\boldsymbol{x}^{k}\right)$.

Osserviamo che, in conseguenza del lemma 12.1, è sufficiente stabilire la (13.2) per funzioni $u \in C^{\infty}\left(\bar{\Omega}, \partial / \partial n_{i}\right)$.

Assegnata allora $u \in C^{\infty}\left(\bar{\Omega}, \partial / 2 n_{i}\right)$, poniamo $u_{k}=\varphi_{k} u$.

Nel corso di questa dimostrazione denoteremo con $c_{1}, \ldots, c_{12}$ delle costanti positive indipendenti da $u, s$ e $\lambda$.

Dall'ipotesi di forte quasi-ellitticità (13.1) si deduce, con note considerazioni $\left({ }^{24}\right)$, ehe si ha per ogni $\lambda>0$

$$
\underset{<x, q>=N}{\Sigma}\left|D^{x}\left(\sigma^{s-N} \boldsymbol{u}_{k}\right)\right|_{2,0}^{2}+\lambda\left|\sigma^{s-N-\gamma} \boldsymbol{u}_{k}\right|_{2,0}^{2}
$$

(24) Cfr. anche la (12.6). 


$$
\begin{aligned}
& \leq c_{1} \int_{0}\left|\left(A_{0 k}\left(\sigma^{s-N} \boldsymbol{u}_{k}\right)+\lambda \sigma^{s-N-2 \boldsymbol{u}_{k}}\right) \cdot \sigma^{s-N} \boldsymbol{u}_{k}\right| d x \\
& \leq c_{1}\left|\sigma^{N}\left(A_{0 k}\left(\sigma^{s-N} \boldsymbol{u}_{k}\right)+\lambda \sigma^{s-N-2 \Upsilon} \boldsymbol{u}_{k}\right)\right|_{2,0} \cdot\left|\sigma^{s-2 N} \boldsymbol{u}_{k}\right|_{2,0} .
\end{aligned}
$$

Dalla (13.6), con considerazioni analoghe a quelle tenute nel corso della dimostrazione del lemma 12.4, si dednce facilmente che, fissato un intervallo $\left[s^{\prime}, s^{\prime \prime}\right]$, si ha per ogni $s \in\left[s^{\prime}, s^{\prime \prime}\right]$ e per ogni $\lambda>0$

$$
\begin{aligned}
& \underset{<\alpha, q>=N}{\boldsymbol{\sum}}\left|\boldsymbol{D}^{\alpha} \boldsymbol{u}_{k}\right|_{2, s-N}^{2}+\lambda\left|\boldsymbol{u}_{k}\right|_{2, s-N-Y}^{2} \\
& \leq c_{2}\left[\left(\left|A_{0 k} u_{k}+\lambda \sigma^{-2} \boldsymbol{u}_{k}\right|_{2, s}+|s-N| \underset{<\alpha, q><2 N}{\Sigma}\left|D^{\alpha} \boldsymbol{u}_{k}\right|_{2, s+<\alpha, q>-2 N)}\right) \cdot\left|\boldsymbol{u}_{k}\right|_{2, s-2 N}\right. \\
& +|s-N|^{2} \underset{<\alpha, q><N}{\Sigma}\left|D^{\alpha} \boldsymbol{u}_{k}\right|_{2, s+<\alpha, q>-2 N] .}^{2}
\end{aligned}
$$

Da quest' altima relazione, dal lemma 12.5 e dalla (13.3) scritta con $s-N$ in luogo di $s$ segue che si ha, supposto $s^{\prime \prime}<N+1 / 2$,

$$
\begin{array}{r}
\left|\boldsymbol{u}_{k}\right|_{2, s-2 N}+\lambda^{1 / 2}\left|\boldsymbol{u}_{k}\right|_{2, s-N-\gamma} \leq c_{3}\left|A_{0 k} \boldsymbol{u}_{k}+\lambda \sigma^{-2} \gamma \boldsymbol{u}_{k}\right|_{2, s} \\
+c_{4}|s-N| \underset{<\alpha, q><2 N}{\Sigma}\left|D^{\alpha} \boldsymbol{u}_{k}\right|_{2, s+<\alpha, q>-2 N} .
\end{array}
$$

Dalla (13.7) e dalla (12.10) scritta per $\chi=2 N, \tau=q, \beta=2 \gamma$ e con $s-2 N$ in luogo di $s$ si deduce la limitazione

$$
\begin{gathered}
\sum_{<\alpha, q>S_{2 N}}\left|D^{\alpha} \boldsymbol{u}_{k}\right|_{2, s+<\alpha, q>-2 N}+\lambda^{1 / 2}\left|\boldsymbol{u}_{k}\right|_{2, s-N-\gamma} \\
\leq c_{5}\left|A_{0 k} \boldsymbol{u}_{k}+\lambda \sigma^{-2} \boldsymbol{u}_{k}\right|_{2, s}+c_{6}|s-N| \sum_{<\alpha, q><2 N}\left|D^{\alpha} \boldsymbol{u}_{k}\right|_{2, s+<\alpha, q>-2 N},
\end{gathered}
$$

da cui segue che esiste $\left.\eta_{0} \in\right] 0,1 / 2\left[\right.$ tale che per ogni $s \in\left[N-\eta_{0}, N+\eta_{0}\right] \Theta$ per ogni $\lambda>0$ si ha

$$
\sum_{<\alpha, q>\leq 2 N}\left|D^{\alpha} u_{k}\right|_{2, s+<\alpha, q>-2 N}+\lambda^{1 / 2}\left|u_{k}\right|_{2, s-N-Y} \leq c_{7}\left|A_{0 k} u_{k}+\lambda \sigma^{-2} u_{k}\right|_{2, s} .
$$

D'altra parte dalla (13.5), dall'ipotesi che i coefficienti $a_{\mu}(x) \in L_{N+\gamma-<\mu, q>}^{2}(\Omega)$ per $\langle\mu, q\rangle\langle 2 N$ e dal lemma 7.5 si deduce facilmente che si ha

$$
\begin{aligned}
& \left|\boldsymbol{A}_{0 \hat{k}} \boldsymbol{u}_{k}-A \boldsymbol{u}_{k}\right|_{2, s} \leq \varepsilon \sum_{<\alpha, q>=2 N}\left|D^{\alpha} \boldsymbol{u}_{k}\right|_{2, s}+c_{8} \underset{<\alpha, q><2 N}{\Sigma}\left|D^{\alpha} \boldsymbol{u}_{k}\right|_{2, s+<\alpha, q>-N-\gamma} \\
& \leq \varepsilon \sum_{<\alpha, q>=2 N}\left(\left|D^{\alpha} \boldsymbol{u}_{k}\right|_{2, s}+\left|D^{\alpha} \boldsymbol{u}_{k}\right|_{2, s+N-\gamma}\right)+c(\varepsilon)\left|\boldsymbol{u}_{k}\right|_{2, s-N-\gamma} .
\end{aligned}
$$


Da quest' ultima relazione, dalla (13.8), dalla condizione $\gamma \leq N$ e dalle ipotesi relative ai coefficienti $a_{\mu}(x)$ segue che si ha

$$
\begin{aligned}
& \sum_{<\alpha, q>\leq 2 N}\left|D^{\alpha} \boldsymbol{u}_{k}\right|_{2, s+<\alpha, q>-2 N}+\lambda^{1 / 2}\left|\boldsymbol{u}_{k}\right|_{2, s-N-\gamma} \\
& \leq c_{9}\left(\left|A u_{k}+\lambda \sigma^{-2} \boldsymbol{u}_{k}\right|_{2, s}+\left|\boldsymbol{u}_{k}\right|_{2, s-N-Y}\right) \\
& \leq c_{10}\left(\left|A u+\lambda \sigma^{-2} u\right|_{2, s}+\sum_{<\mu, q>\leq 2 N} \underset{\alpha<\mu}{\Sigma}\left|a_{\mu} D^{x} u\right|_{2, s}+|u|_{2, s-N-\gamma}\right) \\
& \leq c_{11}\left(\left|A u+\lambda \sigma^{-2} \boldsymbol{u}\right|_{2, s}+\sum_{<\alpha, q><2 N}^{\Sigma}\left(\left|D^{x} u\right|_{2, s}+\left|D^{x} u\right|_{2, s+<\alpha, q>-N-\gamma}\right)+|\boldsymbol{u}|_{2, s-N-\gamma}\right) .
\end{aligned}
$$

D'altra parte osserviamo che dall'ipotesi

segue che si ha

$$
\gamma \geq \sup _{<\alpha, q><2 N}<\alpha, q>-N
$$

$$
\left|D^{x} \boldsymbol{u}\right|_{2, s} \leq c_{12}\left|D^{x} \boldsymbol{u}\right|_{2, s+<\alpha, q>-N-\gamma} \quad \text { per }<\alpha, q><2 N .
$$

Tenendo presente che $u=\sum_{k=1}^{P} u_{k}$, da quest' ultima relazione, dalla (13.9) e dal lemma 7.5 si deduce con ovvie considerazioni la tesi.

Sussiste inoltre il seguente teorema di unicità :

Teorema 13.2. - Sia A un operatore della forma (12.26) e supponiamo che siano verificate le ipotesi del lemma 12.4, l'ipotesi $\left.a_{2}\right)$ e le condizioni $a_{k k}(x) \in C^{|k|}(\bar{\Omega}-S), D^{k-\alpha} a_{k k}(x) \in L_{2 N-<x+h, q>}^{\infty}(\Omega)$ per $\alpha<k$.

Esistono due costanti $\lambda_{0}>0$ e $\left.\eta_{0} \in\right] 0,1 / 2\left[\right.$ tali che per ogni $\lambda \geq \lambda_{0}$, per ogni $s \in\left[N-\eta_{0}, N+\eta_{0}\right]$ e per ogni $u \in W_{s}^{2 N}\left(\Omega, \partial / 2 n_{i}\right)$ sussiste la limitazione

$$
\|u\|_{W_{s}^{2 N(\Omega)}} \leq c\left\|A u+\lambda \sigma^{-\beta} u\right\|_{L_{s}^{2(0)}},
$$

dove c è una costante indipendente $d a u, s$ e $\lambda$.

Alla dimostrazione di questo teorema premettiamo il seguente

Lemma 13.2. - Siano verificate le ipotesi del lemma 12.4 e la condizione $a_{2}$ ).

Esistono due costanti $\lambda_{0}>0$ e $\left.s_{0} \in\right] 0,1 / 2[$ tali che per ogni numero com. plesso $\lambda$, con Re $\lambda \geq \lambda_{0}$, per ogni $s \in\left[-s_{0}, s_{0}\right]$ e per ogni $u \in H_{0, s}^{N}(\Omega)$ sussiste la limitazione

$$
\|u\|_{W_{s}^{N}(0)}^{2} \leq c R e\left(a_{s}(u, u)+\lambda \int_{\hat{\Omega}} \sigma^{-\beta} u \cdot \overline{\sigma^{2 s} u} d x\right),
$$

dove c è una costante positiva indipendente da $u, s$ e $\lambda$. 
Infatti, dai lemmi 12.4 e 13.1 si deduce evidentemente che esiste una costante $\left.s_{0} \in\right] 0,1 / 2\left[\right.$ tale che per ogni $s \in\left[-s_{0}, s_{0}\right]$ e per ogni $u \in H_{0, s}^{N}(\Omega)$ si ha

$$
\|u\|_{W_{s}^{N}}^{2}(\Omega) \leq c \operatorname{Re} a_{s}(u, u)+c_{0}\|u\|_{2, s-\beta / 2}^{2},
$$

dove $c$ e $c_{0}$ sono due costanti positive indipendenti da $u$ es.

Dalla (13.12) si dednce evidentemente la tesi.

Dimostrazione del teorema 13.2. - La tesi è un'opvia conseguenza dei lemmi $12.3,12.5,13.2$ e dell'osservazione che per ogni $u \in W_{s}^{2 N}\left(\Omega, \partial / \partial n_{i}\right)$ riesce

$$
\begin{gathered}
\operatorname{Re}\left(a_{s-N}(u, u)+\lambda \int_{0} \sigma^{-\beta} u \cdot \overline{\sigma^{2(s-N)} u} d x\right) \\
=\operatorname{Re} \int_{\mathbf{0}}\left(A u+\lambda \sigma^{-\beta} u\right) \cdot \overline{\sigma^{2(s-N)} u} d x \leq\left|A u+\lambda \sigma^{-\beta} u\right|_{2, s} \cdot|u|_{2, s-2 N} .
\end{gathered}
$$

\section{4. - Teoremi di esistenza.}

Consideriamo ora il problema

$$
u \in W_{s}^{2 N}\left(\Omega, \partial / \partial n_{i}\right)
$$

$$
A u+\lambda \sigma^{-2} \gamma=f \text { in } \Omega, \quad f \in L_{s}^{2}(\Omega)
$$

dove $\gamma$ \& un fissato numero reale tale che

$$
\sup _{<\alpha, q><2 N}<\alpha, q>-N \leq \gamma<N
$$

Sussiste il seguente

Teonema 14.1. - Nelle stesse ipotesi del teorema 13.1, esistono due costanti $\lambda_{0}>0$ e $\left.\eta_{0} \in\right] 0,1 / 2\left[\right.$ tali che per ogni $\lambda \geq \lambda_{0}$, per ogni $s \in\left[N-\eta_{0}, N+\eta_{0}\right]$ e per ogni $f \in L_{s}^{2}(\Omega)$ il problema (14.1) è univocamente risolubile.

Inoltre, per ogni $\lambda$ complesso e per ogni $s \in\left[N-\eta_{0}, N+\eta_{0}\right],(14.1)$ è un problema a indice e il suo indice è nullo.

Cominciamo con l'osservare che la tesi è vera nell'ulteriore ipotesi che i coefficienti $a_{\mu}(x) \in C^{\infty}(\bar{\Omega})$. Infatti allora la prima parte del teorema si dimostra, tenendo conto del teorema di unicita 13.1 , con ragionamenti analo. 
ghi a quelli tenuti per stabilire il teorema 8.3 , e la seconda parte si ottiene poi come conseguenza del lemma 7.1 di [33] e del lemma 7.6.

Supponendo ora verificate le sole ipotesi del teorema, assegnamo, per ogni $\mu$ tale che $\langle\mu, q\rangle=2 N$, una successione $\left\{a_{\mu}^{(k)}(x)\right\}$ di funzioni equicon. tinue ed equilimitate di classe $C^{\infty}(\bar{\Omega})$. uniformemente convergente in $\bar{\Omega}$ ad $a_{\mu}(x)$, e, per ogni $\mu$ tale che $<\mu, q><2 N$, una successione $\left\{a_{\mu}^{(k)}(x)\right\}$ di funzioni di classe $C^{\infty}(\vec{\Omega})$ ed equilimitate in $L_{N+\gamma-<\mu, q}^{\infty}>(\Omega)$, convergente quasi ovunque in $\Omega$ ad $a_{\mathfrak{u}^{2}}(x)$.

Supponiamo inoltre, ciò che è lecito, che si abbia

$$
R e \underset{<\mu, q>=2 N}{\sum} a_{\mu}^{(k)}(x) \xi^{\mu} \geq a_{0} \sum_{i=1}^{n} \xi_{i}^{2 N_{i}} \quad \forall x \in \bar{\Omega} \quad \text { } \quad \forall \xi \in R^{n}
$$

dove $a_{0}$ è una costante positiva indipendente da $x, \xi$ e $k$.

Posto

$$
A_{k}=\sum_{<\mu, q>\leq 2 N}^{\Sigma} a_{k \beta}^{(k)}(x) D^{\mu},
$$

consideriamo il problema

$$
\begin{aligned}
& u \in W_{s}^{2 N}\left(\Omega, \partial / \partial n_{i}\right), \\
& A_{k} u+\lambda \sigma^{-2 \curlyvee} u=f \text { in } \Omega .
\end{aligned}
$$

Da quanto premesso e dal teorema 13.1 di unicità segue che esistono due costanti $\lambda_{0}>0$ e $\left.\eta_{0} \in\right] 0,1 / 2\left[\right.$, indipendenti da $k$, tali che per ogni $\lambda \geq \lambda_{0}$, per ogni $s \in\left[N-\eta_{0}, N+\eta_{0}\right]$ e per ogni $f \in L_{s}^{2}(\Omega)$ il problema (14.3) ammette un' unica soluzione $u_{k}$ la quale soddisfa la limitazione

$$
\left\|u_{b}\right\|_{W_{s}^{2 N}}^{2 N} \leq c\|f\|_{L_{s}^{2}(\Omega)}
$$

dove $c$ è una costante indipendente da $k$.

Tenuto conto del lemma 7.6, dalla (14.4) segue che da $\left\{u_{k}\right\}$ si può estrarre una successione debolmente convergente in $W_{s}^{2 N}(\Omega)$ e fortemente convergente in $W_{\alpha}^{j}(\Omega)$, per ogni $j \in J(9 K)$ e $<2 N$ e per ogni $\alpha>s+j-2 N$, verso nna funzione $u \in W_{s}^{2 N}(\Omega)$, la quale, evidentemente, riesce una soluzione del problema (14.1).

Da tali considerazioni, dal teorema 13.1 di unicita, dal lemma 7.1 di [33] e dal lemma 7.6 si deduce la tesi.

Consideriamo inoltre il problema

$$
\begin{aligned}
& u \in W_{s}^{2 N}\left(\Omega, \partial / \partial n_{i}\right), \\
& A u+\lambda \sigma^{-\beta} u=f \text { in } \Omega, \quad f \in L_{s}^{2}(\Omega),
\end{aligned}
$$


dove $\beta$ è un fissato numero reale tale che

$$
0 \leq \beta<2 N \text {. }
$$

Vogliamo dimostrare il seguente

Teorema 14.2, - Nelle stesse ipotesi del teorema 13.2, esistono due costanti $\gamma_{0}>0$ e $\left.\gamma_{0} \in\right] 0,1 / 2\left[\right.$ tali che per ogni numero complesso $\lambda$ con Re $\lambda \geq \gamma_{0}$, per ogni $s \in\left[N-\eta_{0}, N+\eta_{0}\right]$ e per ogni $f \in L_{s}^{2}(\Omega)$ il problema (14.5) è univocamente risolubile.

Inoltre $(14.5) \grave{e}$, per ogni $\lambda$ complesso e per ogni $s \in\left[N-\eta_{0}, N+\eta_{0}\right]$, un problema a indice e il suo indice è nullo.

Infatti, con ragionamenti del tipo di quelli tenuti per dedurre dal teorema 13.1 la tesi del teorema 14.1 , si deduce dal teorema 13.2 che esistono due costanti $\lambda_{1}>0$ e $\left.\eta_{1} \in\right] 0,1 / 2\left[\right.$ tali che per ogni $\lambda \geq \lambda_{1}$, per ogni $s \in\left[N-\eta_{1}, N+\eta_{1}\right]$ e per ogni $f \in L_{s}^{2}(\Omega)$ il problema $(14.5)$ è univocamente risolubile e che inoltre (14.5) è, per ogni $\lambda$ complesso e per ogni $s \in\left[N-\eta_{1}\right.$, $N+\eta_{1}$ ], un problema a indice di indice nullo.

D'altra parte, tenuto conto del lemma 12.5, si ha che una soluzione di (14.5) è anche una soluzione del problema

$$
\begin{aligned}
& u \in H_{0, s-N}^{N}(\Omega), \\
& a_{s-N}(u, v)+\lambda \int_{\mathbf{Q}} \sigma^{-B} u \cdot \overline{\sigma^{2(s-N)} v} d x=\int_{\Omega} f \cdot \overline{\sigma^{2(s-N)} v} d x \quad \forall v \in H_{0, s-N}^{N}(\Omega),
\end{aligned}
$$

e quindi dal lemma 13.2 segue che per ogni $\lambda$ complesso con Re $\lambda \geq \lambda_{0}$ e per ogni $s \in\left[N-s_{0}, N+s_{0}\right]$ (dove $\lambda_{0}$ e $s_{0}$ sono le costanti definite dal lemma 13.2) il problema (14.5) ammette al più una soluzione.

Da tali considerazioni si deduce in modo ovvio la tesi.

Osservazione 14.1. - Supponiamo che i coefficienti $a_{\mu}(x)$ di $A$ siano sufficientemente regolari (ad es., supponiamo che sia soddisfatta l'ipotesi $\mathfrak{H}_{6}$ )).

Allora dal lemma 11.3 segue che, se sono anche verificate le ipotesi del teorema 14.1, le condizioni di compatibilità per il problema (14.1) sono date dall'ortogonalità, secondo il prodotto scalare in $L^{2}(\Omega)$, di $f$ alle soluzioni del problema omogeneo

$$
\begin{aligned}
& v \in W_{-s+2 N}^{2 N}\left(\Omega, \partial / \partial \boldsymbol{n}_{i}\right), \\
& A^{*} v+\bar{\lambda} \sigma^{-2 \gamma} v=0 \text { in } \Omega .
\end{aligned}
$$


Analogamente si ha che se, oltre alle suddette ipotesi di regolarità sui coefficienti $a_{\mu}(x)$ di $A$, sono verificate le ipotesi del teorema 14.2 , le condizioni di compatibilita per il problema (14.5) sono date dall'ortogonalità, secondo il prodotto scalare in $L^{2}(\Omega)$, di $f$ alle soluzioni del problema omogeneo

$$
\begin{aligned}
& v \in W_{-s+2 N}^{2 N}\left(\boldsymbol{\Omega}, \partial / \partial n_{i}\right), \\
& A^{*} v+\bar{\lambda} \sigma^{-\beta} v=0 \text { in } \Omega .
\end{aligned}
$$

Osservaztone 14.2. - Supponendo verificate le ipotesi del teorema 13.1, consideriamo il problema

$$
\begin{aligned}
& u \in W_{s}^{2 N}\left(\Omega, \partial / \partial n_{i}\right), \\
& A u=f \text { in } \Omega, \quad f \in L_{s}^{2}(\Omega) .
\end{aligned}
$$

Dal teorema 14.1 segue che esiste una costante $\left.\eta_{0} \in\right] 0,1 / 2[$ tale che il problema (14.8) ha nucleo di dimensione finita per ogni $s \leq N+\eta_{0}$ ed è un problema ad indice, con indice nullo, per ogni $s \in\left[N-\eta_{0}, N+\eta_{0}\right]$.

Vogliamo ricercare in un esempio (che riferiremo al caso ellittico, cioè al caso $t=1$ ) l'insieme dei valori di $s$ per cui il problema ha nucleo di dimensione finita $\theta$ l'insieme dei valori di $s$ per $\dot{i}$ quali il problema è un problema a indice.

Nel piano $R^{2}$, consideriamo il cerchio $\Omega=\left\{(x, y) \mid x^{2}+y^{2}<1\right\}$ e indichiamo con $\rho(x, y)$ la distanza di $(x, y)$ da $\partial \Omega$.

Allora se $A=\Delta=$ operatore di LAPLACE, il problema (14.8) diventa

$$
\begin{aligned}
& u \in W_{s}^{2}(\Omega), \\
& \Delta u=f \text { in } \Omega, \quad f \in L_{s}^{2}(\Omega) .
\end{aligned}
$$

Se $s>3 / 2$, il problema omogeneo associato a (14.9) ha infinite soluzioni linearmente indipendenti. Infatti, utilizzando le coordinate polari $(\boldsymbol{r}, \theta)$, si verifica facilmente che tali, ad es., sono le funzioni

$$
u_{n}(r, \theta)=r^{n}(\cos n \theta+\operatorname{sen} n \theta), \quad n>0 .
$$

Inoltre, osserviamo che l'aggiunto formale di (14.9) è il problema

$$
\begin{aligned}
& v \in W_{-s+2}^{2}(\Omega), \\
& \Delta v=g \text { in } \Omega, \quad g \in L_{-s+2}^{2}(\Omega),
\end{aligned}
$$

e che l'omogeneo associato a tale problema ha infinite soluzioni linearmente indipendenti per $s<1 / 2$. 
D'altra parte, se $s<3 / 2$, da noti risultati (cfr., ad es.: il teorema 2.2 del cap. 6 di [26], l'osservazione 5.1 di [33]) segue ehe una funzione $u \in W_{s}^{2}(\Omega)$ ha traccia nulla su $\partial \Omega$ (si tenga anche presente che nel caso considerato si ha $W_{s}^{2}(\Omega)=H_{0, s}^{2}(\Omega)$ ), nel senso che

$$
\lim _{r \rightarrow 1} \int_{0}^{2 \pi}|u(r, \theta)|^{2} d \theta=0
$$

e quindi una soluzione del problema omogeneo associato a (14.9) è una funzione armonica in $\Omega$ e soddisfacente la (14.11).

Siccome la sola funzione nulla gode di queste nltime proprietà (cfr., ad es., il n. 2 di G. Cimmino [14]], si conclude ohe il problema omogeneo associato a (14.9) per $s<3 / 2$ ammette la sola soluzione nulla.

Analogamente si verifica che per $s>1 / 2$ il problema omogeneo associato a (14.10) ammette la sola soluzione nulla.

Inoltre, tenuto conto dell'osservazione 14.1 , si ha che per ogni $s \in] 1 / 2,3 / 2[$ e per ogni $f \in L_{s}^{2}(\Omega)$ il problema (14.9) è univocamente risolubile.

\section{BIBLIOGRAFIA}

[1] S. Agmon, On the eigenfunctions and on the eigenvalues of general elliptic boundary value problems, Comm. Pure Appl. Math., 15 (1962), pp. 119.147.

[2] - - Lectures on elliptic boundary value problems, Van Nostrand Mathematical Studies, Princeton, 1965.

[3] S. AGMon, A. Dodglis, L. NrRenberg, Estimates near the boundary for solutions of elliptic partial differential equations satisfying general boundary conditions, $\mathrm{I}$, Comm. Pure Appl. Math, 12 (1959), pp. 628-727.

[4] L. ARKerXo, On $L^{p}$ estimates for quasi-elliptic boundary problems, Math. Scand., 24 (1969), pp. 141-144

[5] G. C. BARozZI, Sul multi-indice degli operatori quasi-ellittici, Boll. U. M. I., s. III, 19 (1964), pp. 289.299.

[6] - -, Sui polinomi propriamente quasi-ellittici in due variabili, Boll. U.M. I., s. III, 20 (1965), pp. 185-190.

[7] - - Un problema al contorno non omogeneo in un dominio angoloso per equazioni fortemente quasi-ellittiche in due variabili, (I) e (II), Rend. Sem. Mat. Padova, 44 (1970), pp. 27.63 e pp. 319.337.

[8] A. CAVALLUCOI, Sulle proprietd̀ differenziali delle soluzioni delle equazioni quasi-ellittiche, Ann, di Mat., 67 (1965), pp. 143-167.

[9] - - Sulle proprietà differenziali delle soluzioni delle equazioni quasi-ellittiche relati. vamente a domini normali, Boll. U. M. I., s. III, 19 (1964), pp. $465-477$.

[10] - - Alcuni teoremi di tracce, Atti Sem. Mat. Fis. Modena, 15 (1966), pp. 137.157. 
[11] - - Costruzione di un rilevamento per la traccia iperpiana dello spazio $H_{\mu}\left(R^{n}\right)$, Boll. U. M. I., s. III, 22 (1967), pp. 491.496.

[12] - - Sulla regolarità delle soluzioni delle equazioni quasi-ellittiche in un semispazio, Atti Sem. Mat. Fis. Modena, 17 (1968), pp. 1.18.

[13] - - Sulla regolaritỏ delle soluzioni delle equazioni quasi-ellittiche non lineari, Rend. di Mat., 1 (1968), pp. 385̃425.

[14] G. CImmino, Nuovo tipo di condiaioni al contorno e nuovo metodo di trattazione per it problema generalizzato di Dirichlet, Rend. Ciro. Mat. Palermo, 61 (1938), pp. 177.221.

[15] G. Geymonat, P. Gris nard, Problemi ai limiti lineari ellitici negli spazi ai Sobolev con peso, Le Matematiche, 22 (1967), pp. $212-249$.

[16] E. Grustr, Equazioni quasi-ellittiche e spazi $\mathcal{L}^{p, \theta}(\Omega$, o), (I) e (II), Ann. di Mat., 75 (1967), pp. 313-353; Ann. Sc. Norm. Sup. Pisa, 2L (1967), pp. 353-372.

[17] P. Grisvard, Équations différentielles abstraites, Ann. scient. É. Norm. Sup, 2 (1969), pp. 311.395 .

[18] L. Hörmander, Linear partial differential operators, Springer-Verlag, 1963

[19] M. Itano, On a trace theorem for the space $H^{\mu}\left(R^{z}\right)$, J. Sci. Hiroshima Univ., 30 (1966), pp. 11.29.

[20] J.L. Lions, E. Magenes, Problèmes aux limites non homogènes et applications, Vol. I e II, Dunod Éditeur, Paris, 1968.

[21] T. Matsuzawa, On quasi-elliptic boundary problems, Trans. Amer. Math. Soc., 133 (1968), pp. 241.265.

[22] - -, Sur les équations quasi-elliptiques et les classes de Gevrey, Bull. Soc. Math. France, 96 (1968), pp. 243-263.

[23] C. Mrranda, Teoremi di unicitò in domini non limitati e teoremi di liouville per le soluzioni dei problemi al contorno relativi alle equazioni ellittiche, Ann. di Mat, õ (1962), pp. 189.212.

[24] - - , Partial differential equations of elliptic type, Springer-Verlag, 1970.

[25] J. NrČAs, sur une méthode pour résoudre les équations anx dérivées partielles du type elliptique, voisine de la variationnelle, Ann. Sc. Norm. Sup. Pisa, 16 (1962), pp. 305.326.

[26] - - Les Méthodes directes en théorie des équations elliptiques, Masson et $\mathrm{C}^{\mathrm{ie}}$, Ǵditeurs, Paris; Academia, Éditeurs, Prague, 1967.

[27] M. PAGNi, Problemi al contorno per una certa classe di equazioni lineari alle derivate parziali, Atti Sem. Mat. Fis. Modena, 13 (1964), pp. 119-164.

[28] C. Parentr, Problema di Divichlet in un semispazio relativo ad una classe di operatori quasi-ellittici, Boll. U.M. I, s IV, 3 (1970), pp. 104121.

[39] - - Valutazioni a priori e regolarità per soluzioni di equazioni quasi-ellittiche, in corso di stampa sui Rend. Sem. Mat. Padova.

[30] J. PEetre, A proof of the hypoellipticity of formally hypoelliptic differential operators, Comm. Pure Appl. Math, 14 (1961), pp. $737-744$.

[31] B. PINI, Su un problema tipico relativo a una certa classe di equazioni ipoellittiche, Atti Acc. Sei. Ist. Bologna, s. 12, 1 (1964), pp. 1.26.

[32] - -, Sulla rappresentazione delle soluzioni delle equazioni quasi-ellittiche in una regione angolosa, Ann. di Mat., 80 (1968), pp. 359.372.

[33] M. Trors, Problemi ellitici con dati singolari, Ann. di Mat., 83 (1969), pp. 363.407.

[34] - - Ulteriori contributi allo studio dei problemi ellitici con dati singolari, Ric. di Mat., 19 (1970), pp. 9.25. 
[35] - - Ulteriori contributi alla teoria degli spazi di Sobolev non isotropi, Ric. di Mat., 20 (1971), pp. 90-117.

[36] L. R. Vorevioh, Proprietò locali delle soluzioni dei sistemi quasi-ellittici (in russo), Mat. Sbornik, 59 (1962), pp. 3.52.

[37] L. R. Volevion, B.P. PandeyakH, Alouni spazi di funzioni generalizate e teoremi di immersione (in russo), Usp. Mat. Nauk, 20 (1965), pp. 3.74; trad. inglese: Russian Math. Surveys; 20 (1965), pp. 1.73. 